Supporting Information

\title{
Breaking the Symmetry of a Meso Compound by Isotopic Substitution: \\ Synthesis and Stereochemical Assignment of Monodeuterated cis-Perhydroazulene
}

\author{
Fumito Saito, ${ }^{1}$ Dennis Gerbig, ${ }^{1}$ Jonathan Becker, ${ }^{2}$ and Peter R. Schreiner ${ }^{1 *}$ \\ ${ }^{1}$ Institute of Organic Chemistry, Justus Liebig University \\ Heinrich-Buff-Ring 17, 35392 Giessen, Germany \\ ${ }^{2}$ Institute of Inorganic and Analytical Chemistry, Justus Liebig University \\ Heinrich-Buff-Ring 17, 35392 Giessen, Germany
}

prs@uni-giessen.de

\section{Table of Contents}

Synthesis and Characterization of New Compounds

NMR Spectra

X-ray Crystallographic Analysis .

Optical Rotatory Dispersion Experiments...

Vibrational Circular Dichroism Experiments. S46

Computational Details .

References. S50 


\section{Synthesis and Characterization of New Compounds}

\section{General Experimental Methods}

All air- and water sensitive reactions were carried out under $\mathrm{N}_{2}$ atmosphere using standard Schlenk techniques. Reactions that required heating were carried out using an oil bath. All chemicals and common organic solvents (ACS or HPLC grade) were used as supplied. Thin layer chromatography (TLC) was performed plastic-backed silica gel 60 coated with a fluorescence indicator and visualized by UV quenching and/or staining with $\mathrm{KMnO}_{4}\left(0.75 \mathrm{~g} \mathrm{KMnO}_{4}, 5 \mathrm{~g} \mathrm{~K}_{2} \mathrm{CO}_{3}, 70 \mathrm{mg} \mathrm{NaOH}, 100 \mathrm{~mL}\right.$ $\mathrm{H}_{2} \mathrm{O}$ ) followed by warming with a heat gun. Flash column chromatography was performed under a forced-flow of $\mathrm{N}_{2}$ using Merck silica gel 60 (0.040-0.063 mm particle size). NMR spectra were recorded on Bruker AV400 or AV600 spectrometers. Chemical shifts ( $\delta$ ) are given in ppm relative to residual solvent peaks. Data for ${ }^{1} \mathrm{H}$ NMR are reported as follows: chemical shift (multiplicity, coupling constants where applicable, number of hydrogens). IR spectra were recorded on an ALPHA spectrometer from a Bruker Optics and only peaks diagnostic for major functional groups are reported in frequency of absorption $\left(\mathrm{cm}^{-1}\right)$. High-resolution mass spectra (HRMS) were measured on a Bruker microTOF spectrometer, and the mass for the found ions are given in $\mathrm{m} / \mathrm{z}$ units. Optical rotations were measured on a polarimeter P-2000 from JASCO at the sodium D line with a $50 \mathrm{~mm}$ optical path length and reported as $[\alpha]_{\mathrm{D}}^{25}$ (concentration $(\mathrm{g} / 100 \mathrm{~mL})$, solvent). Melting points (m.p.) were measured on a Krüss Optronic melting point meter KSP1N and are reported uncorrected.

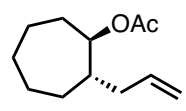

$(-)-4$

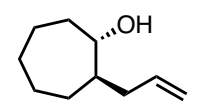

$(-)-3$

To maximize yields and enantiopurities of both (-)-4 and (-)-3, we performed the enzymatic resolution three times consecutively as described below.

First resolution: To a solution of $( \pm)-3^{1}(21.3 \mathrm{~g}, 0.14 \mathrm{~mol}, 1.0$ equiv) and vinyl acetate $(25.6 \mathrm{~mL}, 0.28$ mol, 2.0 equiv) in $\mathrm{Et}_{2} \mathrm{O}(500 \mathrm{~mL})$ was added Amano lipase from Pseudomonas fluorescens $(14.9 \mathrm{~g})$. The mixture was stirred at room temperature for $24 \mathrm{~h}$ and filtered through Celite (rinsed with $\mathrm{Et}_{2} \mathrm{O}$ ). The filtrate was concentrated under reduced pressure, and the residual oil was purified by flash column chromatography (hexanes/EtOAc $50: 1 \rightarrow 5: 1)$ to give less polar $(-)-\mathbf{4}(7.9 \mathrm{~g}, 29 \%$ ) as a colorless oil and more polar (-)-3 (14.3 g, 67\%) as a pale yellow oil.

Second resolution: To a solution of (-)-3 obtained from the first resolution $(14.3 \mathrm{~g}, 93 \mathrm{mmol}, 1.0$ equiv) and vinyl acetate (17.2 mL, $186 \mathrm{mmol}, 2.0$ equiv) in $\mathrm{Et}_{2} \mathrm{O}(330 \mathrm{~mL})$ was added Amano lipase 
from Pseudomonas fluorescens (10.0 g). The mixture was stirred at room temperature for $20 \mathrm{~h}$ and filtered through Celite (rinsed with $\mathrm{Et}_{2} \mathrm{O}$ ). The filtrate was concentrated under reduced pressure, and the residual oil was purified by flash column chromatography (hexanes/EtOAc 50:1 $\rightarrow 5: 1$ ) to give less polar (-)-4 (2.5 g, 9\% based on ( \pm )-3) as a colorless oil and more polar (-)-3 (11.0 g, 52\% based on ( \pm -3) as a yellow oil.

Third resolution: To a solution of (-)-3 obtained from the second resolution (11.0 g, $71 \mathrm{mmol}, 1.0$ equiv) and vinyl acetate (13.2 mL, $142 \mathrm{mmol}, 2.0$ equiv) in $\mathrm{Et}_{2} \mathrm{O}(250 \mathrm{~mL})$ was added Amano lipase from Pseudomonas fluorescens $(7.7 \mathrm{~g})$. The mixture was stirred at room temperature for $18 \mathrm{~h}$ and filtered through Celite (rinsed with $\mathrm{Et}_{2} \mathrm{O}$ ). The filtrate was concentrated under reduced pressure, and the residual oil was purified by flash column chromatography (hexanes/EtOAc 50:1 $\rightarrow 5: 1$ ) to give less polar (-)-4 (0.88 g, 4\% based on ( $( \pm)-3)$ as a colorless oil and more polar (-)-3 (10.0 g, 47\% based on ( \pm )-3) as a pale yellow oil.

The resolution processes above provided (-)-4 (11.3 g, 41\%) and (-)-3 (10.0 g, 47\%).

Characterization data for (-)-4: IR (thin film) 1735, 1640, $1246 \mathrm{~cm}^{-1} ;[\alpha]_{\mathrm{D}}{ }^{25}=-25.8\left(\mathrm{c} 0.65, \mathrm{CDCl}_{3}\right)$; ${ }^{1} \mathrm{H} \mathrm{NMR}\left(\mathrm{CDCl}_{3}, 400 \mathrm{MHz}\right) \delta 5.81-5.67(\mathrm{~m}, 1 \mathrm{H}), 5.03-4.99(\mathrm{~m}, 1 \mathrm{H}), 4.99-4.95(\mathrm{~m}, 1 \mathrm{H}), 4.68$ $(\mathrm{dt}, J=8.8,5.2 \mathrm{~Hz}, 1 \mathrm{H}), 2.20-2.11(\mathrm{~m}, 1 \mathrm{H}), 2.03(\mathrm{~s}, 3 \mathrm{H}), 1.98-1.88(\mathrm{~m}, 1 \mathrm{H}), 1.79-1.56(\mathrm{~m}, 7 \mathrm{H})$, 1.55 - $1.34(\mathrm{~m}, 3 \mathrm{H}), 1.30-1.19(\mathrm{~m}, 1 \mathrm{H}) ;{ }^{13} \mathrm{C} \mathrm{NMR}\left(\mathrm{CDCl}_{3}, 101 \mathrm{MHz}\right) \delta 170.7,136.7,116.3,78.5$, 43.5, 38.5, 32.9, 28.9, 28.6, 26.7, 22.5, 21.5; HRMS (ESI) $m / z$ : $[\mathrm{M}+\mathrm{Na}]^{+}$Calcd for $\mathrm{C}_{12} \mathrm{H}_{20} \mathrm{NaO}_{2}$ 219.1355; Found 219.1358.

Characterization data for (-)-3: $[\alpha]_{\mathrm{D}}{ }^{25}=-3.8\left(\mathrm{c} 0.55, \mathrm{CDCl}_{3}\right) ;{ }^{1} \mathrm{H} \mathrm{NMR}\left(\mathrm{CDCl}_{3}, 400 \mathrm{MHz}\right) \delta 5.90-$ $5.77(\mathrm{~m}, 1 \mathrm{H}), 5.10-4.98(\mathrm{~m}, 2 \mathrm{H}), 3.54-3.46(\mathrm{~m}, 1 \mathrm{H}), 2.41-2.31(\mathrm{~m}, 1 \mathrm{H}), 2.09-1.98(\mathrm{~m}, 1 \mathrm{H})$, $1.83-1.54(\mathrm{~m}, 7 \mathrm{H}), 1.52-1.36(\mathrm{~m}, 4 \mathrm{H}), 1.29-1.14(\mathrm{~m}, 1 \mathrm{H}) ;{ }^{13} \mathrm{C} \mathrm{NMR}\left(\mathrm{CDCl}_{3}, 101 \mathrm{MHz}\right) \delta 137.6$, $116.2,76.5,47.0,39.3,36.5,28.82,28.80,26.9,22.3$. These NMR spectroscopic data matched with those reported previously. ${ }^{2}$

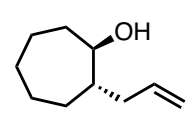

(+)-3

To a solution of (-)-4 (10.4 g, 53 mmol, 1.0 equiv) in $\mathrm{MeOH}(200 \mathrm{~mL})$ was added NaOMe (17.2 g, $0.32 \mathrm{~mol}, 6.0$ equiv). The mixture was stirred at room temperature for $14 \mathrm{~h}$. The solvent was mostly evaporated under reduced pressure. The residue was partitioned between $\mathrm{Et}_{2} \mathrm{O}$ and $\mathrm{H}_{2} \mathrm{O}$, and the 
aqueous phase was extracted with $\mathrm{Et}_{2} \mathrm{O}(2 \mathrm{x})$. The combined organic phases were dried over $\mathrm{Na}_{2} \mathrm{SO}_{4}$, filtered and concentrated under reduced pressure to give (+)-3 (8.3 g, quant) as a pale yellow oil, which was used for the next step without further purification. For analytical purpose, $250 \mathrm{mg}$ of crude (+)-3 was purified by flash column chromatography (hexanes/EtOAc 5:1) to give $245 \mathrm{mg}$ of pure (+)3: $[\alpha]_{\mathrm{D}}{ }^{25}=+4.5\left(\mathrm{c} 0.55, \mathrm{CDCl}_{3}\right)$.

Determination of enantiopurity of the resolved alcohols (+)-3 and (-)-3

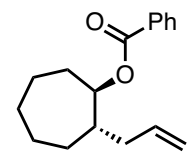

$(-)-\mathbf{S 1}$

To a solution of $(+)-3\left(62 \mathrm{mg}, 0.40 \mathrm{mmol}, 1.0\right.$ equiv) in $\mathrm{CH}_{2} \mathrm{Cl}_{2}(2.0 \mathrm{~mL})$ were added benzoyl chloride (93 $\mu \mathrm{L}, 0.80 \mathrm{mmol}, 2.0$ equiv), followed by TMEDA (122 $\mu \mathrm{L}, 0.80 \mathrm{mmol}, 2.0$ equiv). The mixture was stirred at room temperature for $15 \mathrm{~min}$, directly loaded onto a silica gel column and purified by flash column chromatography (hexanes/EtOAc 25:1) to give (-)-S1 (45 mg, 44\%) as a clear colorless oil: IR (ATR) 1711, 1269, 1109, $708 \mathrm{~cm}^{-1} ;[\alpha]_{\mathrm{D}}{ }^{25}=-50.9\left(\mathrm{c} 0.80, \mathrm{CDCl}_{3}\right) ;{ }^{1} \mathrm{H} \mathrm{NMR}\left(\mathrm{CDCl}_{3}, 400\right.$ $\mathrm{MHz}) \delta 8.10-8.02(\mathrm{~m}, 2 \mathrm{H}), 7.59-7.52(\mathrm{~m}, 1 \mathrm{H}), 7.48-7.41(\mathrm{~m}, 2 \mathrm{H}), 5.84-5.70(\mathrm{~m}, 1 \mathrm{H}), 5.07-$ $4.92(\mathrm{~m}, 3 \mathrm{H}), 2.30-2.19(\mathrm{~m}, 1 \mathrm{H}), 2.08-1.98(\mathrm{~m}, 1 \mathrm{H}), 1.98-1.82(\mathrm{~m}, 3 \mathrm{H}), 1.81-1.64(\mathrm{~m}, 4 \mathrm{H})$, $1.63-1.43(\mathrm{~m}, 3 \mathrm{H}), 1.40-1.28(\mathrm{~m}, 1 \mathrm{H}) ;{ }^{13} \mathrm{C} \mathrm{NMR}\left(\mathrm{CDCl}_{3}, 101 \mathrm{MHz}\right) \delta 166.1,136.6,132.7,131.0$, 129.5, 128.3, 116.5, 79.0, 43.7, 38.5, 32.8, 29.0, 28.8, 26.7, 22.5; HRMS (ESI) $\mathrm{m} / \mathrm{z}:[\mathrm{M}+\mathrm{Na}]^{+} \mathrm{Calcd}$ for $\mathrm{C}_{17} \mathrm{H}_{22} \mathrm{NaO}_{2}$ 281.1512; Found 281.1513; enantiomeric ratio = 96:4 (NP-HPLC, column: Chiralpak IC; eluent: $n$-hexane/EtOAc 98:2; flow rate: $1.0 \mathrm{~mL} / \mathrm{min}$; detection: $254 \mathrm{~nm}$, retention time: $5.2 \min$ (major), $5.4 \min$ (minor)).

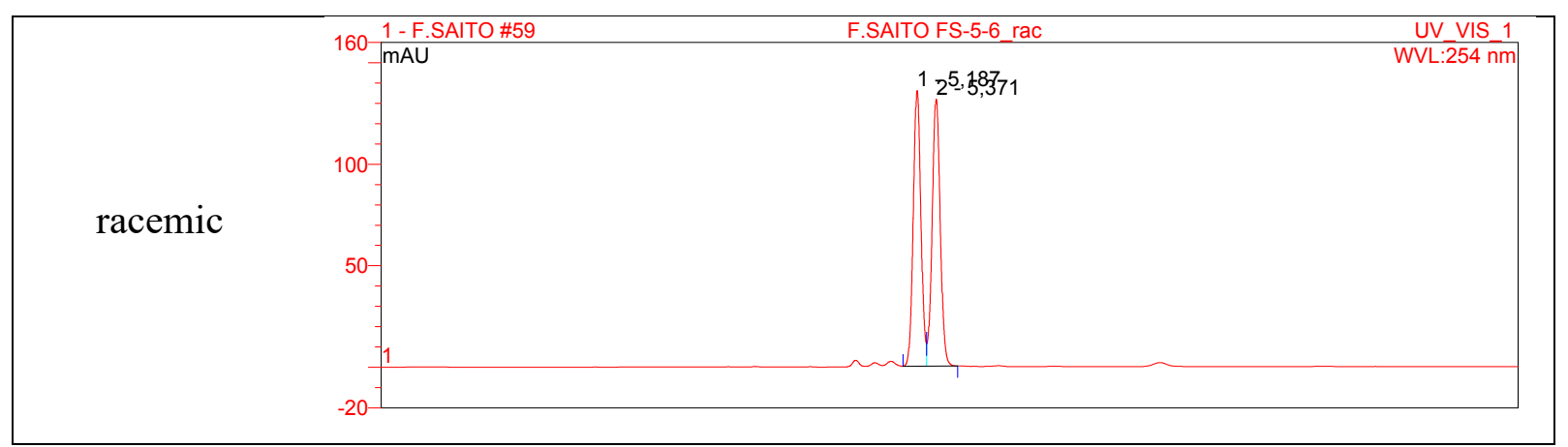



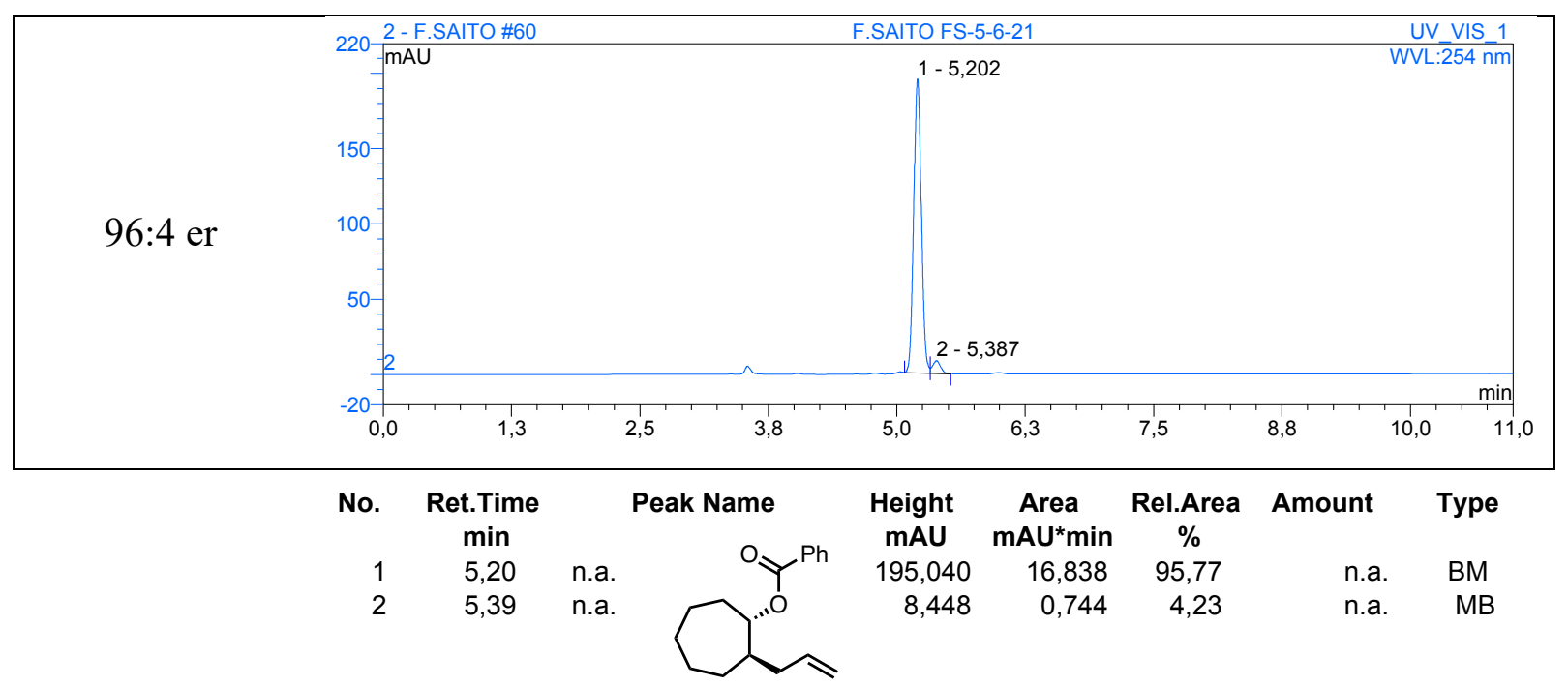

$(+)-\mathbf{S 1}$

Following the same procedure as described above, $(-)-\mathbf{3}$ was transformed into $(+)-\mathbf{S 1}:[\alpha]_{\mathrm{D}}{ }^{25}=+46.8$ (c $\left.0.80, \mathrm{CDCl}_{3}\right)$; enantiomeric ratio $=11: 89$ (NP-HPLC, column: Chiralpak IC; eluent: $n$ hexane/EtOAc 98:2; flow rate: $1.0 \mathrm{~mL} / \mathrm{min}$; detection: $254 \mathrm{~nm}$, retention time: $5.3 \mathrm{~min}$ (minor), 5.5 $\min ($ major)).
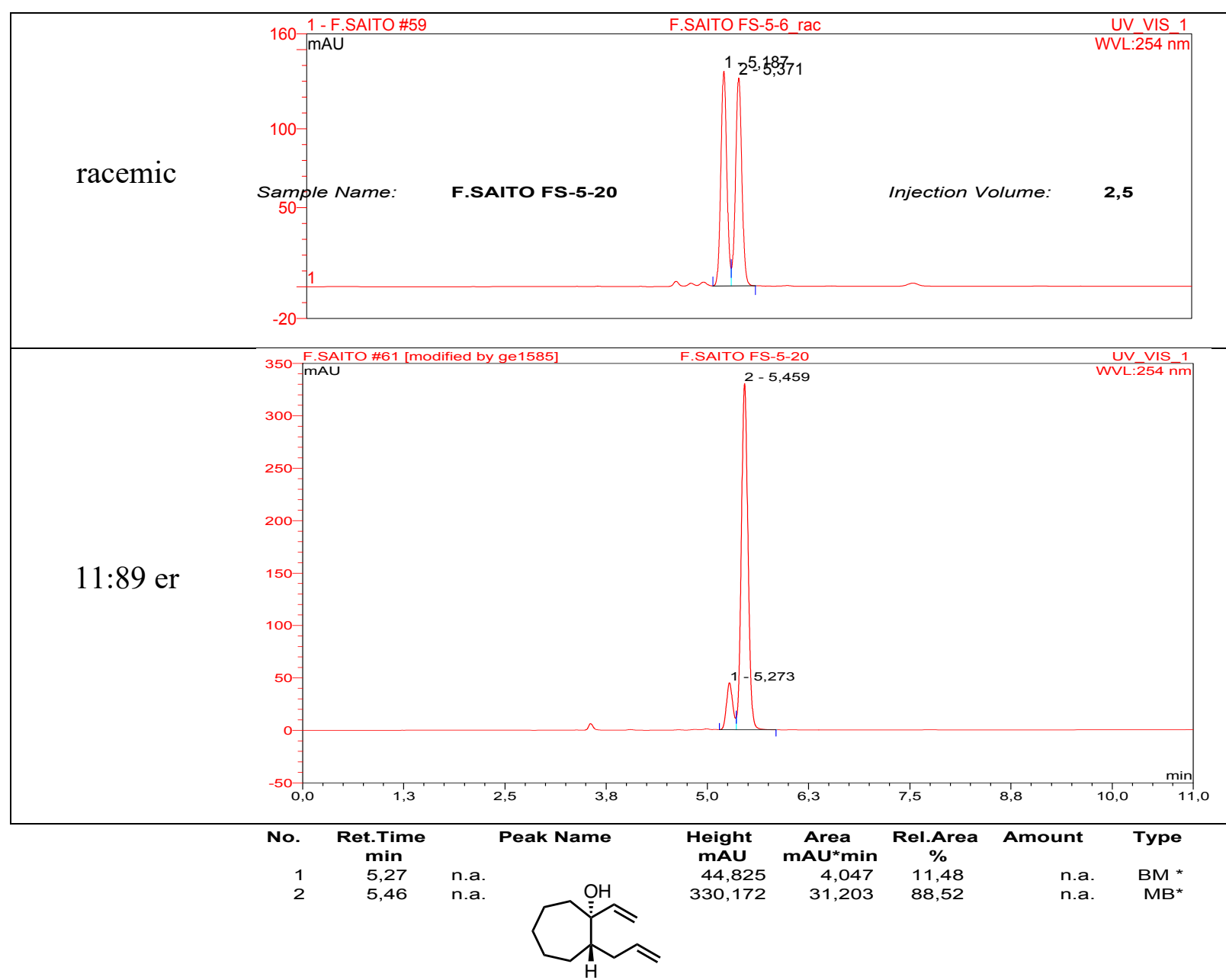

Page S5 of S50 
To a solution of $(+)-3\left(3.0 \mathrm{~g}, 19 \mathrm{mmol}, 1.0\right.$ equiv) in $\mathrm{CH}_{2} \mathrm{Cl}_{2}(100 \mathrm{~mL})$ was added Dess-Martin periodinane ( $9.9 \mathrm{~g}, 23 \mathrm{mmol}, 1.2$ equiv) portionwise at $0{ }^{\circ} \mathrm{C}$. The mixture was allowed to warm to room temperature and stirred at this temperature for $30 \mathrm{~min}$. The mixture was cooled to $0{ }^{\circ} \mathrm{C}$ and a solution of $\mathrm{Na}_{2} \mathrm{~S}_{2} \mathrm{O}_{3} \cdot 5 \mathrm{H}_{2} \mathrm{O}$ in $80 \%$ sat aq $\mathrm{NaHCO}_{3}$ was added to quench the reaction. After stirring for $10 \mathrm{~min}$ at room temperature, the two phases were separated, and the aqueous phase was extracted with $\mathrm{CH}_{2} \mathrm{Cl}_{2}(1 \mathrm{x})$. The combined organic phases were successively washed with sat aq $\mathrm{NaHCO}_{3}$, $\mathrm{H}_{2} \mathrm{O}$, and brine, dried over $\mathrm{Na}_{2} \mathrm{SO}_{4}$, filtered and concentrated under reduced pressure to give a crude ketone (3.0 g, quant) as an orange oil, which was used for the next step without further purification. A $1 \mathrm{M}$ solution of vinylmagnesium bromide ( $48 \mathrm{~mL}, 48 \mathrm{mmol}, 2.5$ equiv) in THF was diluted with THF $(50 \mathrm{~mL})$. To this solution was added the ketone $\left(3.0 \mathrm{~g}, 19 \mathrm{mmol}, 1.0\right.$ equiv) at $0{ }^{\circ} \mathrm{C}$. The mixture was allowed to warm to room temperature and stirred at this temperature for $40 \mathrm{~min}$. The mixture was cooled to $0{ }^{\circ} \mathrm{C}$ and sat aq $\mathrm{NH}_{4} \mathrm{Cl}$ was added to quench the reaction. The two phases were separated, and the aqueous phase was extracted with $\mathrm{Et}_{2} \mathrm{O}(2 \mathrm{x})$. The combined organic phases were washed with brine, dried over $\mathrm{Na}_{2} \mathrm{SO}_{4}$, filtered and concentrated under reduced pressure. The residue was purified by flash column chromatography (hexanes/EtOAc 50:1 $\rightarrow 25: 1)$ to give $(+)-5(3.2 \mathrm{~g}$, $93 \%$ ) as a pale yellow viscous oil: IR (ATR) 3476, 1639, 994, $909 \mathrm{~cm}^{-1}$; $[\alpha]_{\mathrm{D}}^{25}=+40.2(\mathrm{c} 0.65$, $\left.\mathrm{CDCl}_{3}\right) ;{ }^{1} \mathrm{H} \mathrm{NMR}\left(\mathrm{CDCl}_{3}, 400 \mathrm{MHz}\right) \delta 5.95(\mathrm{dd}, \mathrm{J}=17.3,10.8 \mathrm{~Hz}, 1 \mathrm{H}), 5.85-5.72(\mathrm{~m}, 1 \mathrm{H}), 5.21$ $(\mathrm{dd}, \mathrm{J}=17.3,1.3 \mathrm{~Hz}, 1 \mathrm{H}), 5.06(\mathrm{dd}, \mathrm{J}=10.8,1.3 \mathrm{~Hz}, 1 \mathrm{H}), 5.03-5.00(\mathrm{~m}, 1 \mathrm{H}), 5.00-4.96(\mathrm{~m}, 1 \mathrm{H})$, $2.26-2.17(\mathrm{~m}, 1 \mathrm{H}), 1.90-1.78(\mathrm{~m}, 3 \mathrm{H}), 1.75-1.63(\mathrm{~m}, 3 \mathrm{H}), 1.59-1.52(\mathrm{~m}, 2 \mathrm{H}), 1.48-1.23(\mathrm{~m}$, $5 \mathrm{H}) ;{ }^{13} \mathrm{C} \mathrm{NMR}\left(\mathrm{CDCl}_{3}, 101 \mathrm{MHz}\right) \delta 146.4,138.4,115.8,110.8,77.6,47.0,42.0,35.4,29.1,28.5$, 25.9, 21.4; HRMS (ESI) $m / z$ : [M+Na $]^{+}$Calcd for $\mathrm{C}_{12} \mathrm{H}_{20} \mathrm{NaO}$ 203.1406; Found 203.1405. The NMR spectroscopic data matched with those reported previously. ${ }^{3}$

Following the same procedure as described above, $(-)-3$ was transformed into $(-)-5:[\alpha]_{\mathrm{D}} 25=-40.3$ (c $\left.0.65, \mathrm{CDCl}_{3}\right)$.

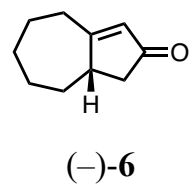

To a solution of (+)-5 (4.0 g, $22 \mathrm{mmol}, 1.0$ equiv) in $\mathrm{CH}_{2} \mathrm{Cl}_{2}(22 \mathrm{~mL})$ under reflux was added Grubbs catalyst second generation ( $90 \mathrm{mg}, 0.11 \mathrm{mmol}, 0.005$ equiv). The mixture was stirred at the same temperature for $30 \mathrm{~min}$, and Grubbs catalyst second generation $(90 \mathrm{mg}, 0.11 \mathrm{mmol}, 0.005$ equiv) was 
further added to the reaction. After stirring at reflux for $30 \mathrm{~min}$, the reaction was cooled to room temperature, and DMSO $(1.6 \mathrm{~mL})$ was added. The mixture was stirred at room temperature for $16 \mathrm{~h}$ and $\mathrm{CH}_{2} \mathrm{Cl}_{2}$ was evaporated under reduced pressure. The residue was purified by flash column chromatography (pentane/ $\left.\mathrm{Et}_{2} \mathrm{O} 25: 2\right)$ to give a metathesis product $(2.9 \mathrm{~g}, 86 \%$ ) as a brown oil.

Note: We found that the metathesis product decomposed when solvent evaporation by a rotary evaporator was performed on a water bath at $40^{\circ} \mathrm{C}$; the bath temperature should not exceed room temperature. In addition, the metathesis product was somewhat unstable under silica gel column chromatography, and repeated purification by column chromatography should be avoided. Due to the instability, the metathesis product was immediately used for the next step without full characterization.

To a suspension of PCC (10.2 g, $47 \mathrm{mmol}, 2.5$ equiv) and NaOAc (6.2 g, $76 \mathrm{mmol}, 4.0$ equiv) in $\mathrm{CH}_{2} \mathrm{Cl}_{2}(130 \mathrm{~mL})$ was added a solution of the metathesis product (2.9 $\mathrm{g}, 19 \mathrm{mmol}, 1.0$ equiv) in $\mathrm{CH}_{2} \mathrm{Cl}_{2}(20 \mathrm{~mL})$. The mixture was stirred at room temperature for $15 \mathrm{~min}$ and filtered through Celite (rinsed with $\mathrm{CH}_{2} \mathrm{Cl}_{2}$ ). The filtrate was concentrated under reduced pressure, and the residue was purified by flash column chromatography (hexanes/EtOAc $4: 1 \rightarrow 3: 1)$ to give $(-)-6(1.3 \mathrm{~g}, 39 \%$ from (+)-5) as a brown oil: IR (thin film) 1688, $1604 \mathrm{~cm}^{-1} ;[\alpha]_{\mathrm{D}}{ }^{25}=-127.8\left(\mathrm{c} 1.0, \mathrm{CDCl}_{3}\right) ;{ }^{1} \mathrm{H}$ NMR $\left(\mathrm{CDCl}_{3}, 400 \mathrm{MHz}\right) \delta 5.87(\mathrm{q}, J=1.6 \mathrm{~Hz}, 1 \mathrm{H}), 2.98-2.90(\mathrm{~m}, 1 \mathrm{H}), 2.79-2.61(\mathrm{~m}, 3 \mathrm{H}), 2.05-1.90$ $(\mathrm{m}, 2 \mathrm{H}), 1.90-1.79(\mathrm{~m}, 1 \mathrm{H}), 1.79-1.65(\mathrm{~m}, 3 \mathrm{H}), 1.52-1.33(\mathrm{~m}, 3 \mathrm{H}) ;{ }^{13} \mathrm{C} \mathrm{NMR}\left(\mathrm{CDCl}_{3}, 101 \mathrm{MHz}\right)$ $\delta$ 209.0, 187.8, 129.7, 44.44, 44.37, 34.3, 32.7, 30.3, 28.5, 26.4. HRMS (ESI) $\mathrm{m} / z$ : $[\mathrm{M}+\mathrm{Na}]^{+}$Calcd for $\mathrm{C}_{10} \mathrm{H}_{14} \mathrm{NaO}$ 173.0937; Found 173.0938; enantiomeric ratio = 98:2 (NP-HPLC, column: Chiralpak IC; eluent: $n$-hexane/isopropanol 80:20; flow rate: $1.0 \mathrm{~mL} / \mathrm{min}$; detection: $210 \mathrm{~nm}$, retention time: 20.5 min (major), 23.5 min (minor)). The NMR spectroscopic data matched with those reported previously. ${ }^{4}$

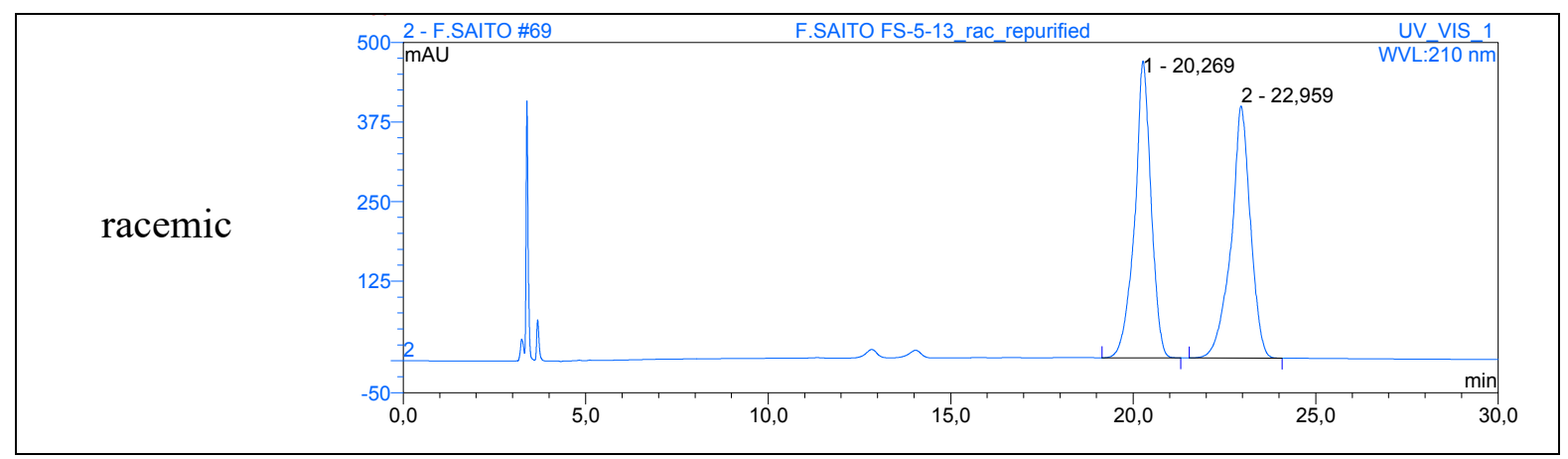




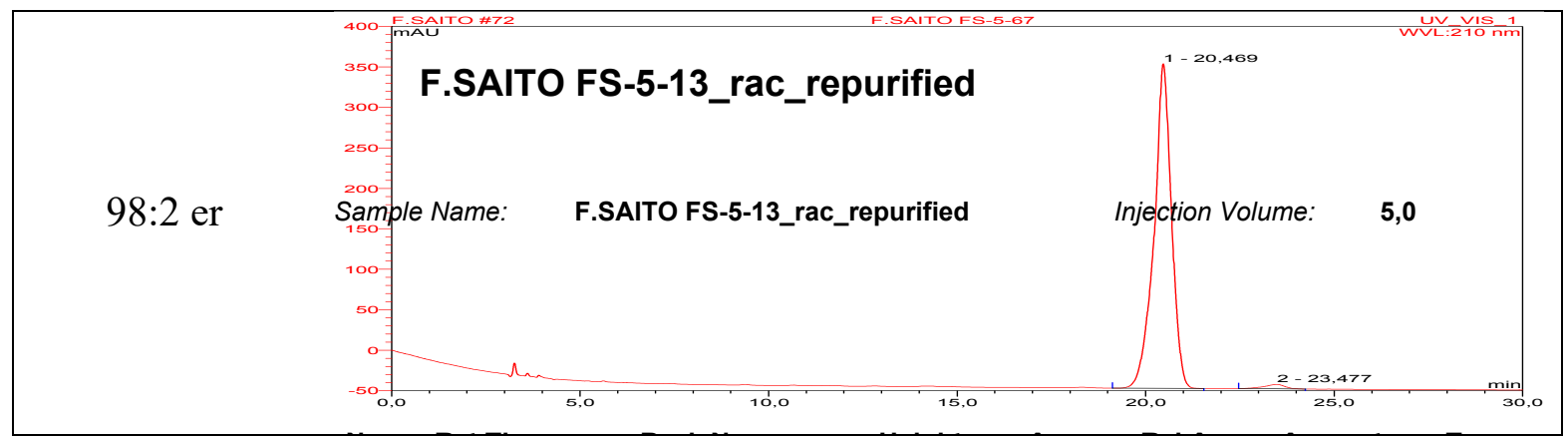

Following the same procedure as described above, $(-)-5$ was transformed into $(+)-6$ : $[\alpha]_{\mathrm{D}}{ }^{25}=+119.6$ (c 1.0, $\mathrm{CDCl}_{3}$ ); enantiomeric ratio $=5: 95$ (NP-HPLC, column: Chiralpak IC; eluent: $n$ hexane/isopropanol 80:20; flow rate: $1.0 \mathrm{~mL} / \mathrm{min}$; detection: $210 \mathrm{~nm}$, retention time: $20.5 \mathrm{~min}$ (minor), $23.0 \mathrm{~min}$ (major)).
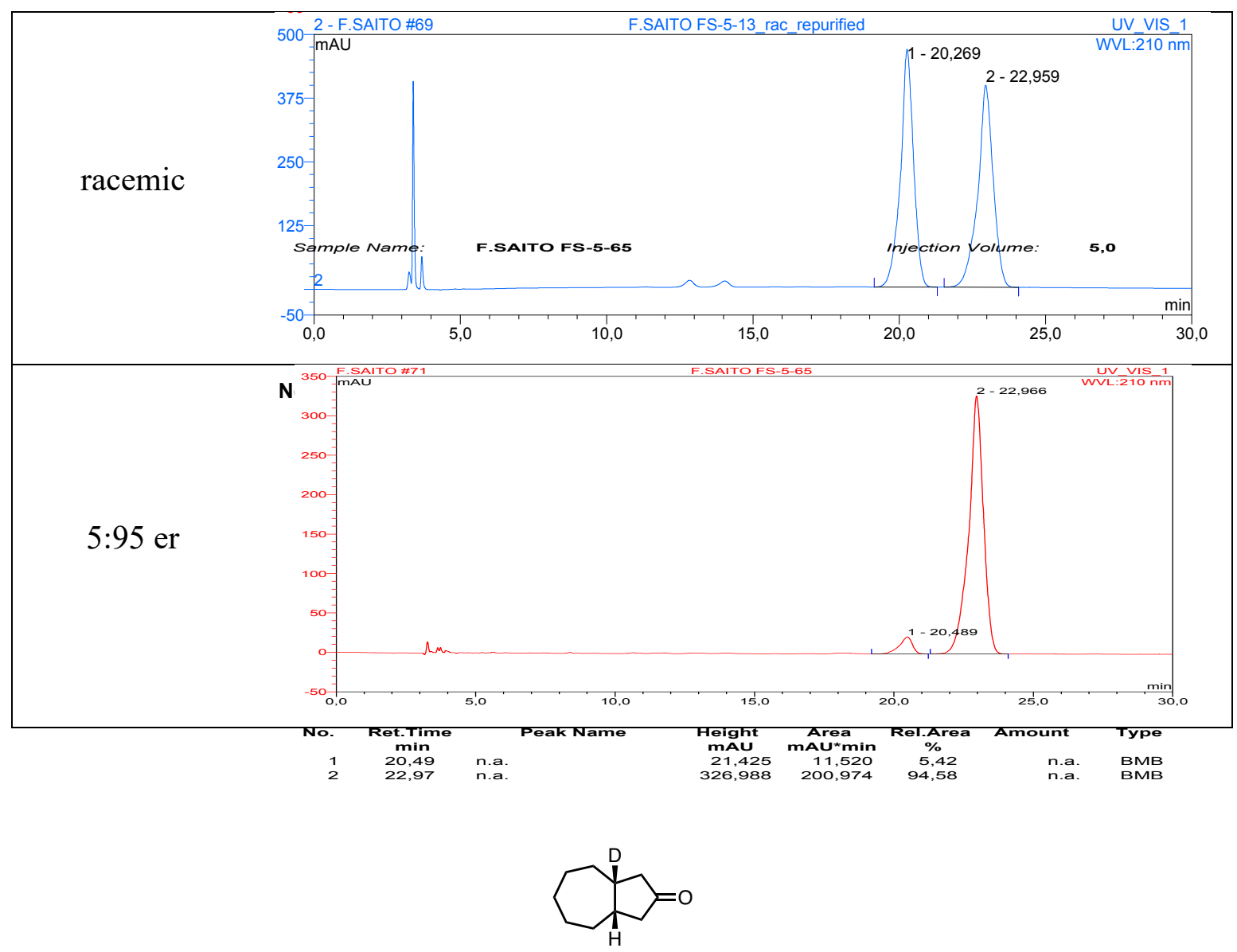

$(+)-d_{1}-8$

To a solution of $\mathrm{NiCl}_{2} \bullet 6 \mathrm{H}_{2} \mathrm{O}\left(2.85 \mathrm{~g}, 12 \mathrm{mmol}, 2.5\right.$ equiv) in $\mathrm{MeOD}(25 \mathrm{~mL})$ was added $\mathrm{NaBD}_{4}(1.0$ g, $24 \mathrm{mmol}, 5.0$ equiv) portionwise at $0{ }^{\circ} \mathrm{C}$. The mixture was stirred at this temperature for $30 \mathrm{~min}$. The supernatant was discarded, and the black precipitates were washed with MeOD (3x $3 \mathrm{~mL})$ and suspended in $\mathrm{MeOD}(25 \mathrm{~mL})$. The reaction was placed under $\mathrm{D}_{2}$ atmosphere (balloon, $1 \mathrm{~atm}$ ), and to 
this was added a solution of (-)-6 (0.72 g, $4.8 \mathrm{mmol}, 1.0$ equiv) in MeOD (3.0 mL). The mixture was stirred at room temperature for $3 \mathrm{~h}$ and filtered through Celite (rinsed with $\mathrm{MeOH}$ ). The filtrate was concentrated at reduced pressure, and the residue was purified by flash column chromatography (hexanes/EtOAc 9:1) to give $7(0.52 \mathrm{~g}, 70 \%)$ as a pale yellow oil.

To a solution of $7(0.52 \mathrm{~g}, 3.4 \mathrm{mmol})$ in $\mathrm{MeOH} / \mathrm{H}_{2} \mathrm{O}(1: 1,26 \mathrm{~mL})$ was added $\mathrm{K}_{2} \mathrm{CO}_{3}(100 \mathrm{mg})$. The reaction was stirred at $50{ }^{\circ} \mathrm{C}$ for $15 \mathrm{~h}$, cooled to room temperature, and diluted with $\mathrm{Et}_{2} \mathrm{O}$. After adding $2 \mathrm{M}$ aq $\mathrm{HCl}(3 \mathrm{~mL})$, the mixture was extracted with $\mathrm{Et}_{2} \mathrm{O}(3 \mathrm{x})$. The combined organic phases were washed with brine, dried over $\mathrm{Na}_{2} \mathrm{SO}_{4}$, filtered, and concentrated under reduced pressure. The residue was re-subjected to the H/D exchange reaction following the same procedure. The crude material was purified by flash column chromatography (hexanes/EtOAc 9:1) to give $(+)-d_{1}-8(0.46 \mathrm{~g}, 62 \%$ from (-)-6) as a pale brown oil: IR (thin film) $1743 \mathrm{~cm}^{-1} ;[\alpha]_{\mathrm{D}}{ }^{25}=+2.7\left(\mathrm{c} 0.50, \mathrm{CDCl}_{3}\right) ;{ }^{1} \mathrm{H} \mathrm{NMR}\left(\mathrm{CDCl}_{3}\right.$, $600 \mathrm{MHz}) \delta 2.48-2.39(\mathrm{~m}, 3 \mathrm{H}), 2.02-1.95(\mathrm{~m}, 2 \mathrm{H}), 1.89-1.78(\mathrm{~m}, 3 \mathrm{H}), 1.78-1.72(\mathrm{~m}, 2 \mathrm{H}), 1.43$ - 1.36 (m, 2H), 1.36 - 1.28 (m, 2H), 1.28 - 1.20 (m, 1H); ${ }^{2} \mathrm{H} \mathrm{NMR}\left(\mathrm{CDCl}_{3}, 92 \mathrm{MHz}\right) \delta 2.45$ (singlet); ${ }^{13} \mathrm{C} \mathrm{NMR}\left(\mathrm{CDCl}_{3}, 151 \mathrm{MHz}\right) \delta 220.1,46.4,46.3,39.6,39.2(\mathrm{t}, J=19.8 \mathrm{~Hz}), 32.3,32.2,31.4,27.83$, 27.82; HRMS (ESI) $m / z$ : [M+Na] $]^{+}$Calcd for $\mathrm{C}_{10} \mathrm{H}_{15} \mathrm{DNaO}$ 176.1156; Found 176.1159.

Following the same procedure as described above, $(+)-6$ was transformed into $(-)-d_{1}-8:[\alpha]_{\mathrm{D}}{ }^{25}=-1.6$ (c $\left.0.50, \mathrm{CDCl}_{3}\right)$.
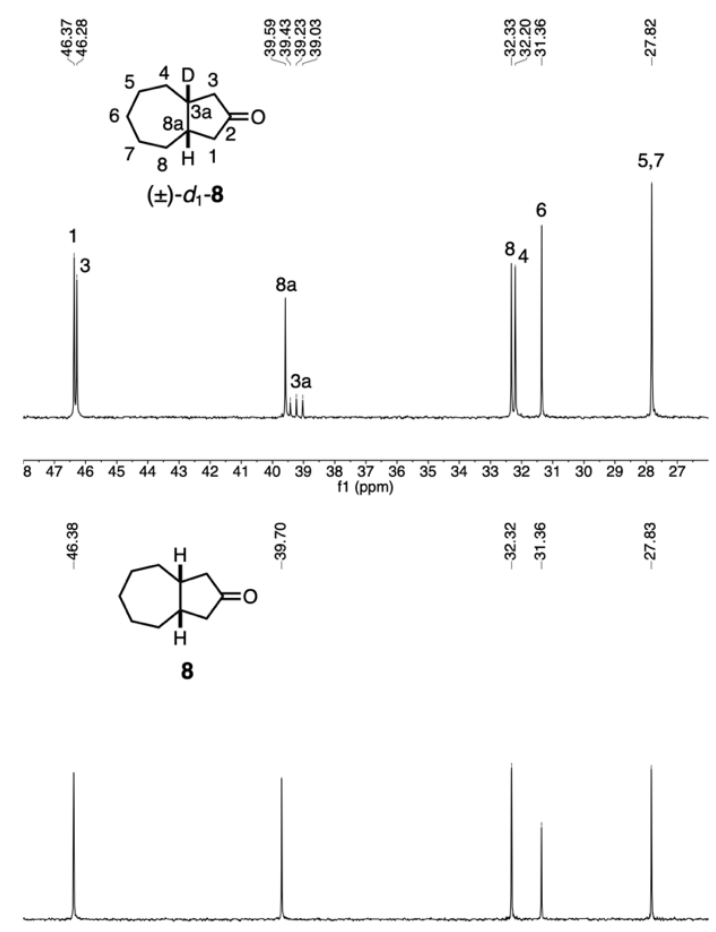

$\begin{array}{lllllllllllllllllllllll}8 & 47 & 46 & 45 & 44 & 43 & 42 & 41 & 40 & 39 & 38 & 37 & 36 & 35 & 34 & 33 & 32 & 31 & 30 & 29 & 28 & 27\end{array}$

Figure S1. ${ }^{13} \mathrm{C}$ NMR spectra of $( \pm)-d_{1}-\mathbf{8}$ (top) and $\mathbf{8}$ (bottom) recorded at $101 \mathrm{MHz}$ in $\mathrm{CDCl}_{3}$. 


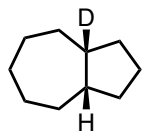

$(3 \mathrm{a} R, 8 \mathrm{a} S)-d_{1}-1$

To a solution of $(+)-d_{1} \mathbf{- 8}(0.51 \mathrm{~g}, 3.3 \mathrm{mmol}, 1.0$ equiv) in $\mathrm{MeOH}(2.2 \mathrm{~mL})$ was added $p$ toluenesulfonyl hydrazide ( $0.68 \mathrm{~g}, 4.3 \mathrm{mmol}, 1.1 \mathrm{equiv})$, and the mixture was stirred at room temperature. Within $5 \mathrm{~min}$, white precipitates started to appear and $\mathrm{MeOH}(1.5 \mathrm{~mL})$ was added to the mixture. After stirring for $1.5 \mathrm{~h}$, the mixture was filtered and the white solid was washed with $\mathrm{MeOH}$ $\left(2 \times 1 \mathrm{~mL}\right.$, pre-cooled at $\left.-20^{\circ} \mathrm{C}\right)$ and dried $(0.87 \mathrm{~g}, 82 \%)$.

The crude tosylhydrazone ( $0.87 \mathrm{~g}, 2.7 \mathrm{mmol}, 1.0$ equiv) was dissolved in sulfolane/DMF (13 mL, 1:1), and to this were added $p-\mathrm{TsOH} \cdot \mathrm{H}_{2} \mathrm{O}(105 \mathrm{mg})$, followed by $\mathrm{NaBH}_{3} \mathrm{CN}(0.68 \mathrm{~g}, 11 \mathrm{mmol}, 4.0$ equiv). The mixture was stirred at $100{ }^{\circ} \mathrm{C}$ for $16 \mathrm{~h}$ and allowed to cool to room temperature. The reaction mixture was partitioned between pentane and $\mathrm{H}_{2} \mathrm{O}$. The aqueous phase was extracted with pentane $(2 \mathrm{x})$, and the combined extracts were washed with brine, dried over $\mathrm{Na}_{2} \mathrm{SO}_{4}$, filtered and concentrated under reduced pressure. The residue was purified by flash column chromatography (pentane) to give $(3 \mathrm{a} R, 8 \mathrm{a} S)-d_{1}-\mathbf{1}(0.11 \mathrm{~g}, 24 \%$ for 2 steps) as a clear colorless liquid: IR (ATR) 2914, 2849, 2131, $1452 \mathrm{~cm}^{-1} ;{ }^{1} \mathrm{H} \mathrm{NMR}\left(\mathrm{CDCl}_{3}, 600 \mathrm{MHz}\right) \delta 1.96-1.89(\mathrm{~m}, 1 \mathrm{H}), 1.78-1.68(\mathrm{~m}, 5 \mathrm{H}), 1.59$ - 1.51 (m, 3H), $1.30-1.09$ (m, 6H), $1.06-1.00$ (m, 2H); ${ }^{2} \mathrm{H} \mathrm{NMR}\left(\mathrm{CDCl}_{3}, 92 \mathrm{MHz}\right) \delta 2.01$ (singlet); ${ }^{13} \mathrm{C} \mathrm{NMR}\left(\mathrm{CDCl}_{3}, 151 \mathrm{MHz}\right) \delta 43.3,42.9(\mathrm{t}, J=19.5 \mathrm{~Hz}), 35.8,35.7,32.9,32.8,31.7,29.71,29.67$, 26.5; HRMS (APCI) $m / z$ : [M-H] $]^{-}$Calcd for $\mathrm{C}_{10} \mathrm{H}_{16} \mathrm{D}$ 138.1398; Found 138.1398.

Following the same procedure as described above, $(-)-d_{1}-\mathbf{8}$ was transformed into $(3 \mathrm{a} S, 8 \mathrm{a} R)-d_{1}-\mathbf{1}$.

\section{Derivatization for X-ray crystallographic analysis}

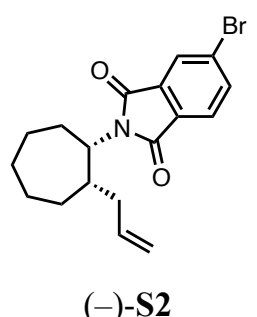

To a solution of (+)-3 (55 mg, $0.36 \mathrm{mmol}, 1.0$ equiv), 4-bromophthalimide (106 mg, $0.47 \mathrm{mmol}, 1.3$ equiv), and $\mathrm{PPh}_{3}$ (123 mg, $0.47 \mathrm{mmol}, 1.3$ equiv) in THF (2.5 mL) was added dropwise a solution of $\operatorname{DEAD}(40 \%$ in toluene, $0.23 \mathrm{~mL}, 0.50 \mathrm{mmol}, 1.4$ equiv). The mixture was stirred at room temperature for $4 \mathrm{~h}$. As TLC indicated the incomplete conversion of (+)-3, 4-bromophthalimide (53 mg, 0.23 
mmol, 0.65 equiv), $\mathrm{PPh}_{3}$ (61 mg, $0.23 \mathrm{mmol}, 0.65$ equiv), and $\mathrm{DEAD}$ (40\% in toluene, $0.11 \mathrm{~mL}, 0.25$ mmol, 0.70 equiv) were further added to the reaction. The mixture was stirred at room temperature for $22 \mathrm{~h}$, directly loaded onto a silica gel column, and purified by flash column chromatography (hexanes/EtOAc 19:1) to give (-)-S2 (120 mg, 92\%) as a pale yellow solid: m.p. $109-110{ }^{\circ} \mathrm{C}$; IR $(\mathrm{KBr}) 1700 \mathrm{~cm}^{-1} ;[\alpha]_{\mathrm{D}}{ }^{25}=-0.5\left(\mathrm{c} 1.0, \mathrm{CDCl}_{3}\right) ;{ }^{1} \mathrm{H} \mathrm{NMR}\left(\mathrm{CDCl}_{3}, 400 \mathrm{MHz}\right) \delta 7.93(\mathrm{~d}, J=1.7 \mathrm{~Hz}$, 1H), $7.83(\mathrm{dd}, J=7.9,1.7 \mathrm{~Hz}, 1 \mathrm{H}), 7.67(\mathrm{~d}, J=7.8 \mathrm{~Hz}, 1 \mathrm{H}), 5.74-5.57(\mathrm{~m}, 1 \mathrm{H}), 4.93-4.81(\mathrm{~m}$, $2 \mathrm{H}), 4.61-4.48(\mathrm{~m}, 1 \mathrm{H}), 2.39-2.24(\mathrm{~m}, 1 \mathrm{H}), 2.12-1.95(\mathrm{~m}, 3 \mathrm{H}), 1.94-1.76(\mathrm{~m}, 5 \mathrm{H}), 1.70-1.60$ (m, 1H), $1.49-1.21(\mathrm{~m}, 3 \mathrm{H}) ;{ }^{13} \mathrm{C} \mathrm{NMR}\left(\mathrm{CDCl}_{3}, 101 \mathrm{MHz}\right) \delta 168.2,167.7,137.3,136.8,133.5,130.4$, 128.7, 126.5, 124.5, 115.9, 55.3, 42.1 36.6, 30.5, 29.8, 29.3, 28.7, 25.6; HRMS (ESI) $m / z:[\mathrm{M}+\mathrm{Na}]^{+}$ Calcd for $\mathrm{C}_{18} \mathrm{H}_{20} \mathrm{BrNNaO}_{2} 384.0569$; Found 384.0571.

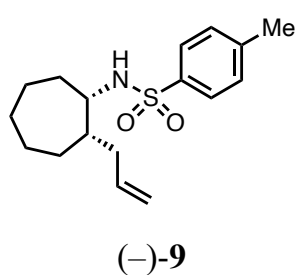

To a suspension of (-)-S2 (89 mg, $0.24 \mathrm{mmol}, 1.0$ equiv) in EtOH (3.0 mL) was added hydrazine monohydrate ( $45 \mu \mathrm{L}, 0.60 \mathrm{mmol}, 2.5$ equiv). The mixture was stirred at reflux for $2 \mathrm{~h}$. Note: the mixture once became a solution upon heating and a white solid precipitated while refluxing for $2 \mathrm{~h}$. The reaction was cooled to room temperature, and conc $\mathrm{HCl}(1 \mathrm{~mL})$ was added. The aqueous solution was washed with $\mathrm{Et}_{2} \mathrm{O}(2 \mathrm{x})$ and basified with solid $\mathrm{NaOH}$ to $\mathrm{pH} 12$. The resulting biphasic mixture was extracted with $\mathrm{Et}_{2} \mathrm{O}(4 \mathrm{x})$. The combined organic extracts were washed with brine, dried over $\mathrm{Na}_{2} \mathrm{SO}_{4}$, filtered and concentrated under reduced pressure to give a crude amine $(33 \mathrm{mg}, 90 \%)$ as a dark brown oil.

To a solution of the crude amine (33 mg, $0.22 \mathrm{mmol}, 1.0$ equiv) in $\mathrm{CH}_{2} \mathrm{Cl}_{2}(1.5 \mathrm{~mL})$ was added $\mathrm{NEt}_{3}$ (61 $\mu \mathrm{L}, 0.43 \mathrm{mmol}, 2.0$ equiv), followed by $p$-toluenesulfonyl chloride (50 mg, $0.24 \mathrm{mmol}, 1.1 \mathrm{equiv}$ ). The mixture was stirred at room temperature for $1 \mathrm{~h}$, directly loaded onto a silica gel column, and purified by flash column chromatography (hexanes/EtOAc 6:1) to give (-)-9 (35 mg, 53\%) as a white solid: m.p. 81-83 ${ }^{\circ} \mathrm{C}$; IR (KBr) 3274, 1331, 1319, $1156 \mathrm{~cm}^{-1} ;[\alpha]_{\mathrm{D}}{ }^{25}=-7.8\left(\mathrm{c} 0.5, \mathrm{CDCl}_{3}\right) ;{ }^{1} \mathrm{H}$ NMR $\left(\mathrm{CDCl}_{3}, 400 \mathrm{MHz}\right) \delta 7.79-7.74(\mathrm{~m}, 2 \mathrm{H}), 7.29(\mathrm{~d}, J=8.0 \mathrm{~Hz}, 2 \mathrm{H}), 5.71-5.57(\mathrm{~m}, 1 \mathrm{H}), 4.99-4.85$ $(\mathrm{m}, 2 \mathrm{H}), 4.51(\mathrm{~d}, \mathrm{~J}=9.0 \mathrm{~Hz}, 1 \mathrm{H}), 3.57-3.48(\mathrm{~m}, 1 \mathrm{H}), 2.42(\mathrm{~s}, 3 \mathrm{H}), 2.07-1.97(\mathrm{~m}, 1 \mathrm{H}), 1.91-1.81$ $(\mathrm{m}, 1 \mathrm{H}), 1.68-1.61(\mathrm{~m}, 1 \mathrm{H}), 1.56-1.38(\mathrm{~m}, 9 \mathrm{H}), 1.31-1.20(\mathrm{~m}, 1 \mathrm{H}) ;{ }^{13} \mathrm{C} \mathrm{NMR}\left(\mathrm{CDCl}_{3}, 101 \mathrm{MHz}\right)$ $\delta 143.1,138.3,137.2,129.6,127.1,116.2,56.2$, 42.9, 37.3, 33.5, 29.0, 27.2, 26.0, 23.4, 21.5; HRMS (ESI) $m / z$ : $[\mathrm{M}+\mathrm{Na}]^{+}$Calcd for $\mathrm{C}_{17} \mathrm{H}_{25} \mathrm{NNaO}_{2} \mathrm{~S} 330.1498$; Found 330.1497. 


\section{NMR Spectra}

Acetate (-)-4

${ }^{1} \mathrm{H}$ NMR (400 MHz, $\left.\mathrm{CDCl}_{3}\right)$

遂

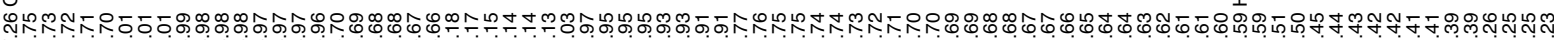

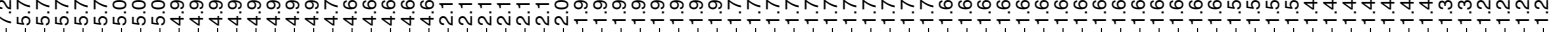
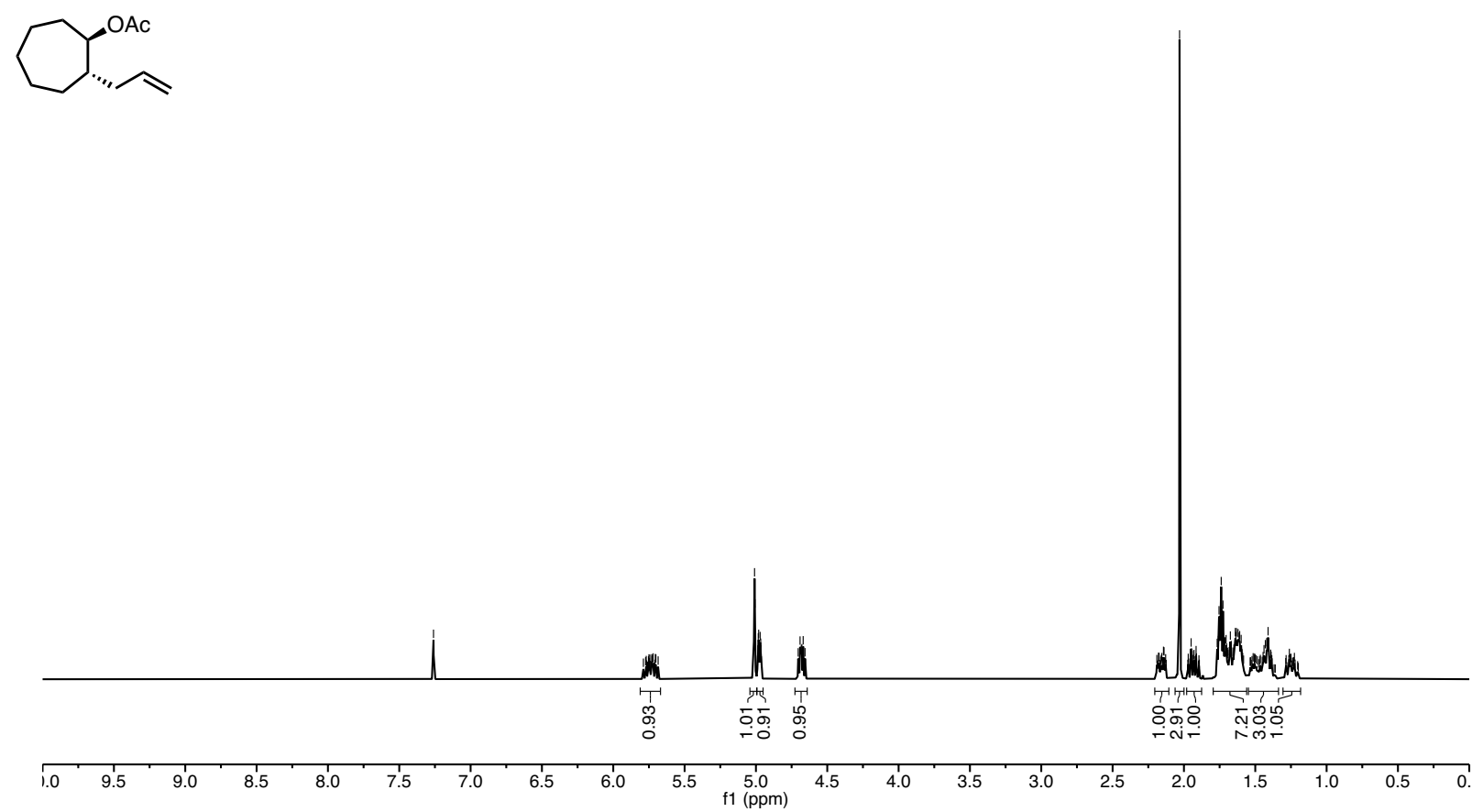

${ }^{13} \mathrm{C}$ NMR (101 MHz, $\left.\mathrm{CDCl}_{3}\right)$<smiles>C=CC[C@H]1CCCCCC1OC(C)=O</smiles>
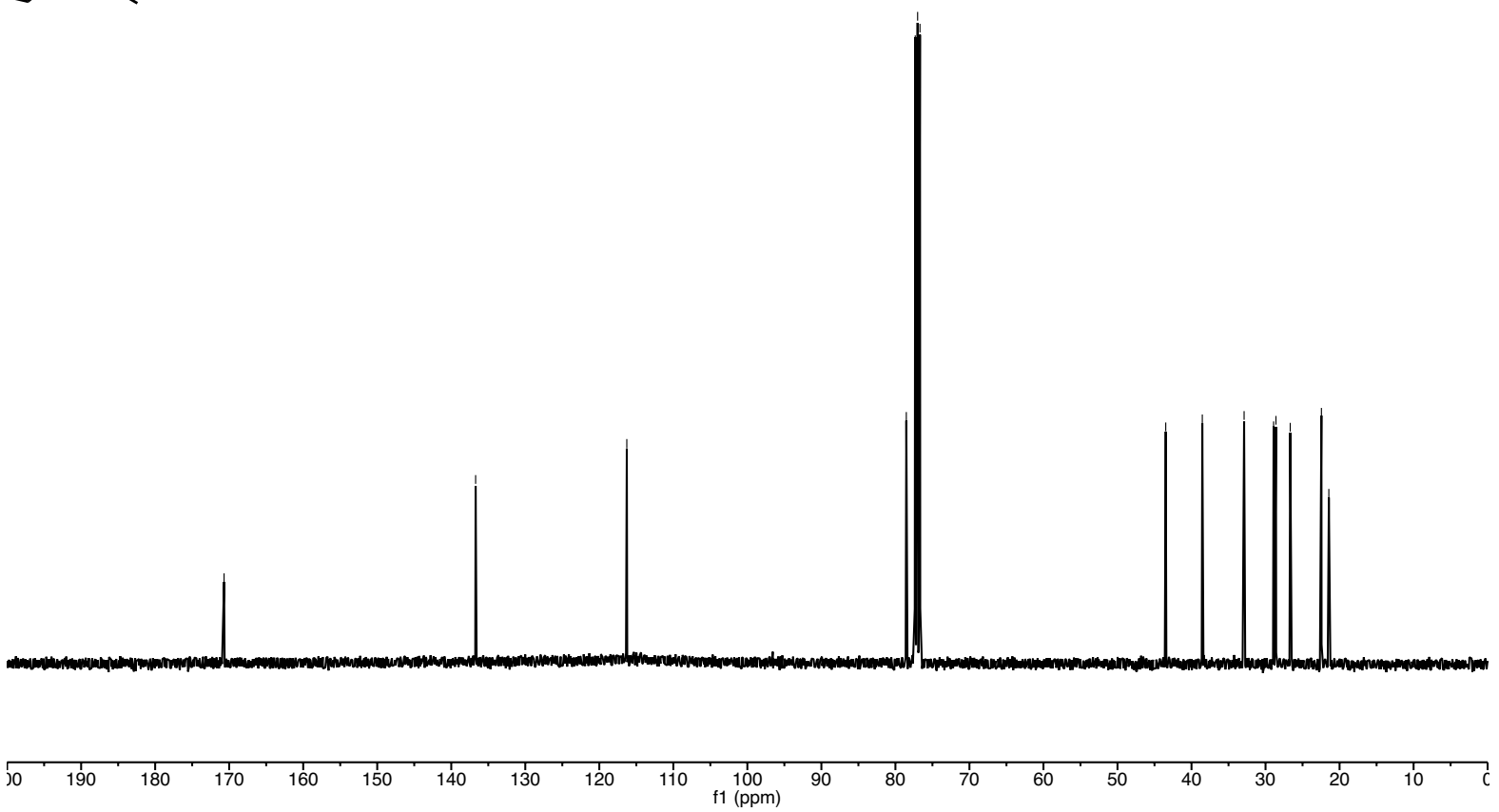
Secondary alcohol (-)-3

${ }^{1} \mathrm{H}$ NMR (400 MHz, $\mathrm{CDCl}_{3}$ )

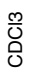

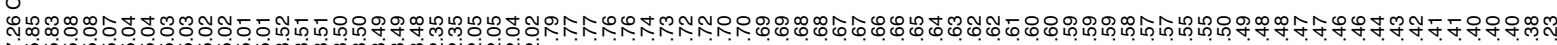

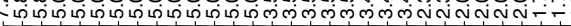<smiles>C=CCC1CCCCC[C@H]1O</smiles>

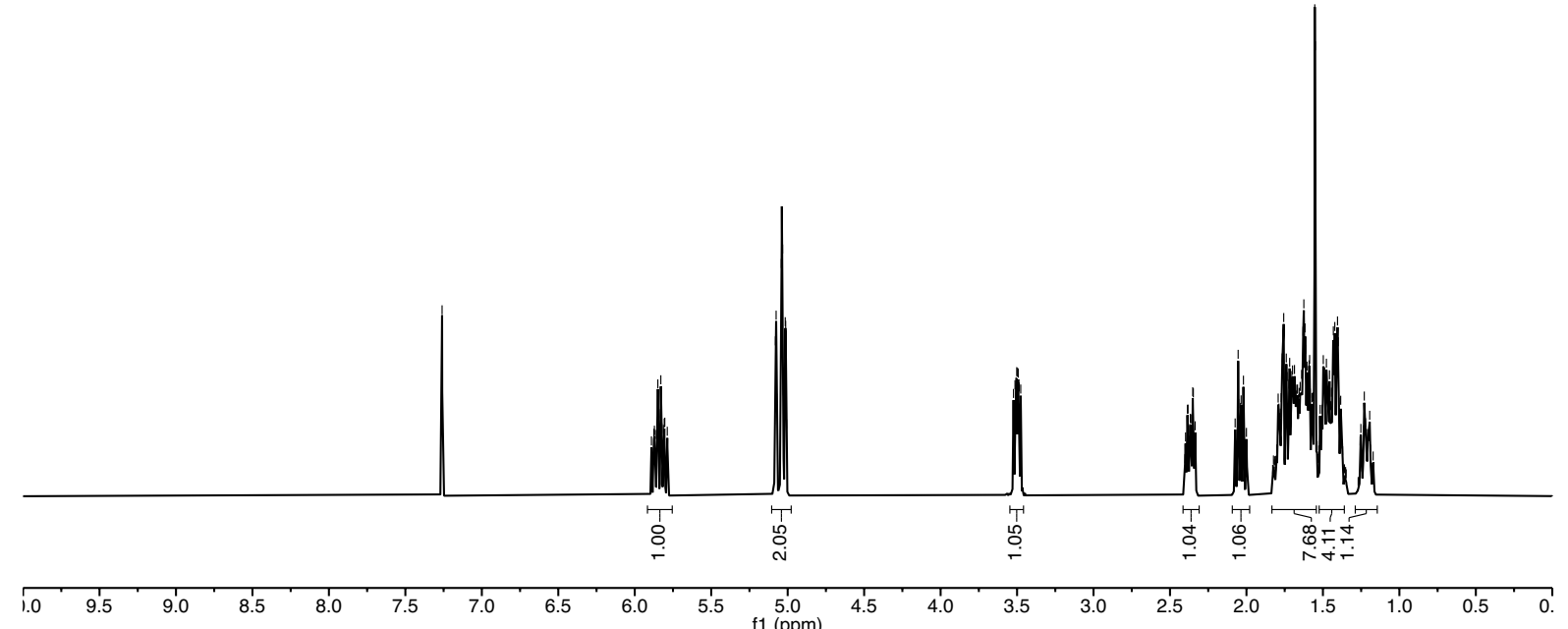

${ }^{13} \mathrm{C}$ NMR (101 MHz, $\left.\mathrm{CDCl}_{3}\right)$

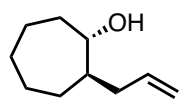

$\stackrel{\bullet}{\stackrel{\bullet}{\stackrel{0}{i}}}$

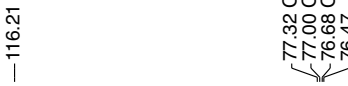

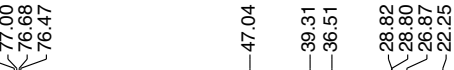

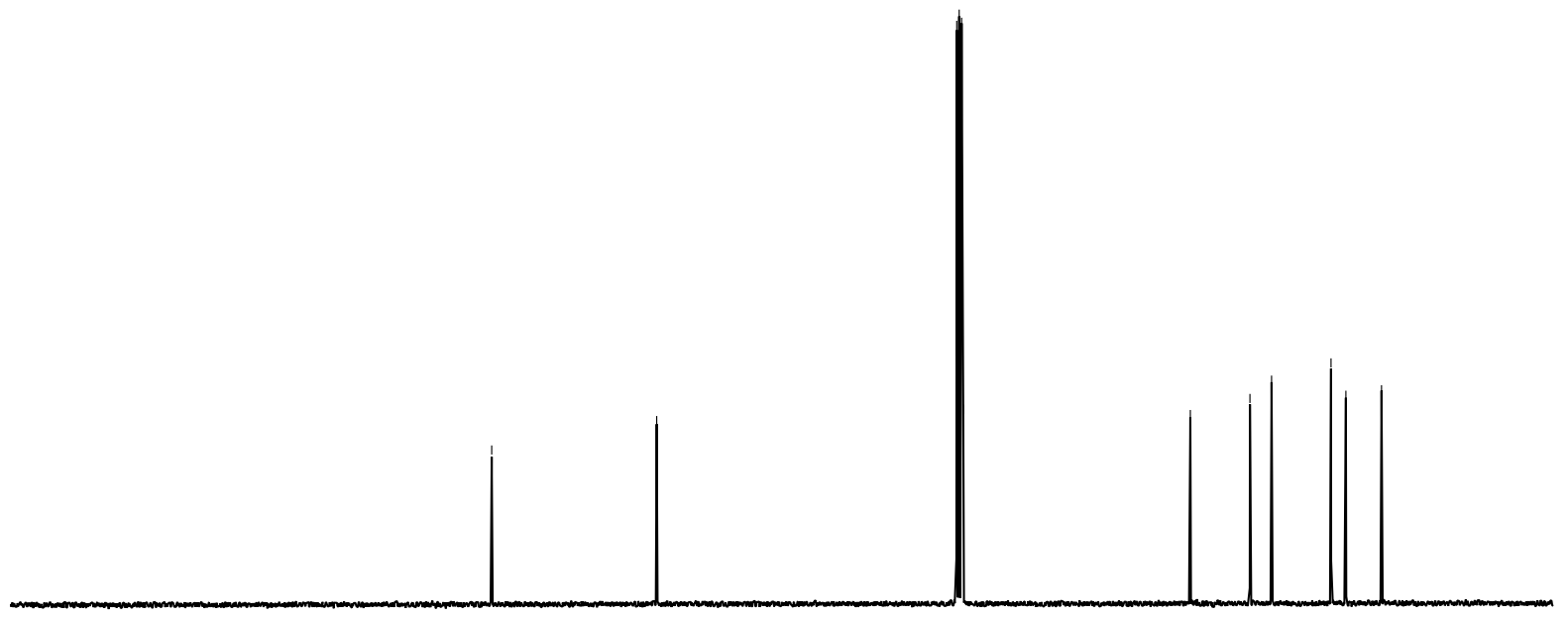

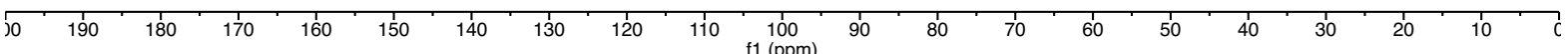


Benzoate (-)-S1

${ }^{1} \mathrm{H}$ NMR (400 MHz, $\mathrm{CDCl}_{3}$ )

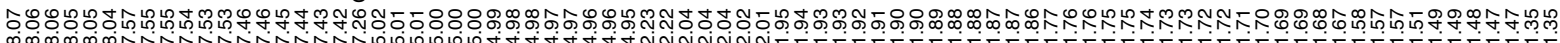<smiles>C=CC[C@H]1CCCCCC1OC(=O)c1ccccc1</smiles>

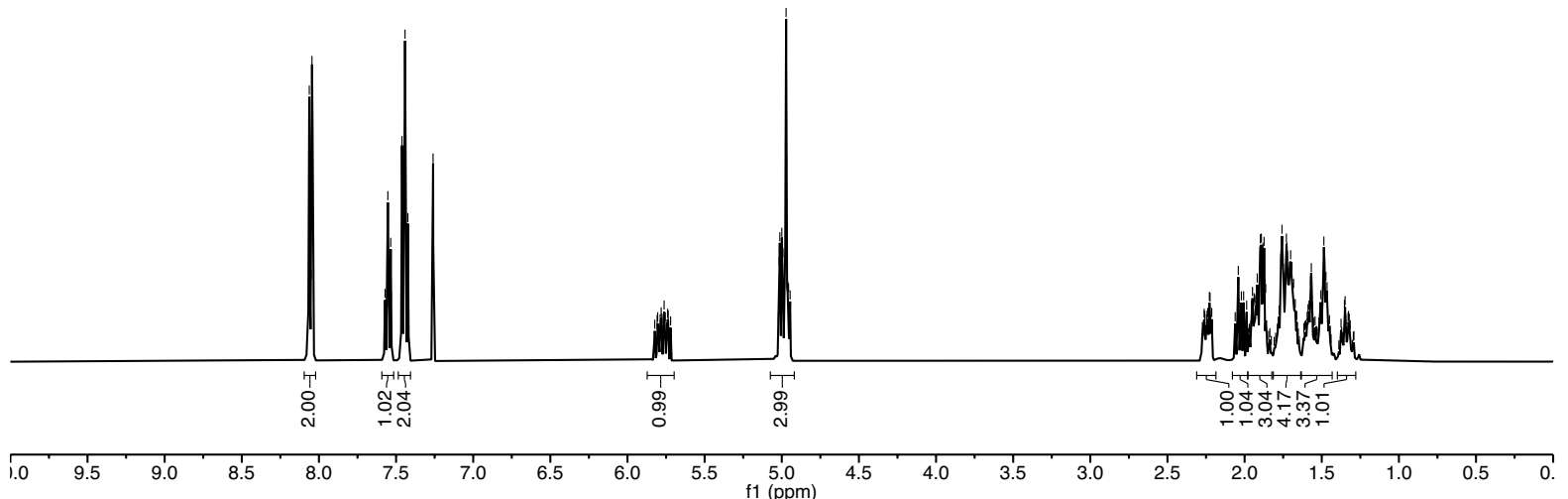

${ }^{13} \mathrm{C}$ NMR (101 MHz, $\left.\mathrm{CDCl}_{3}\right)$

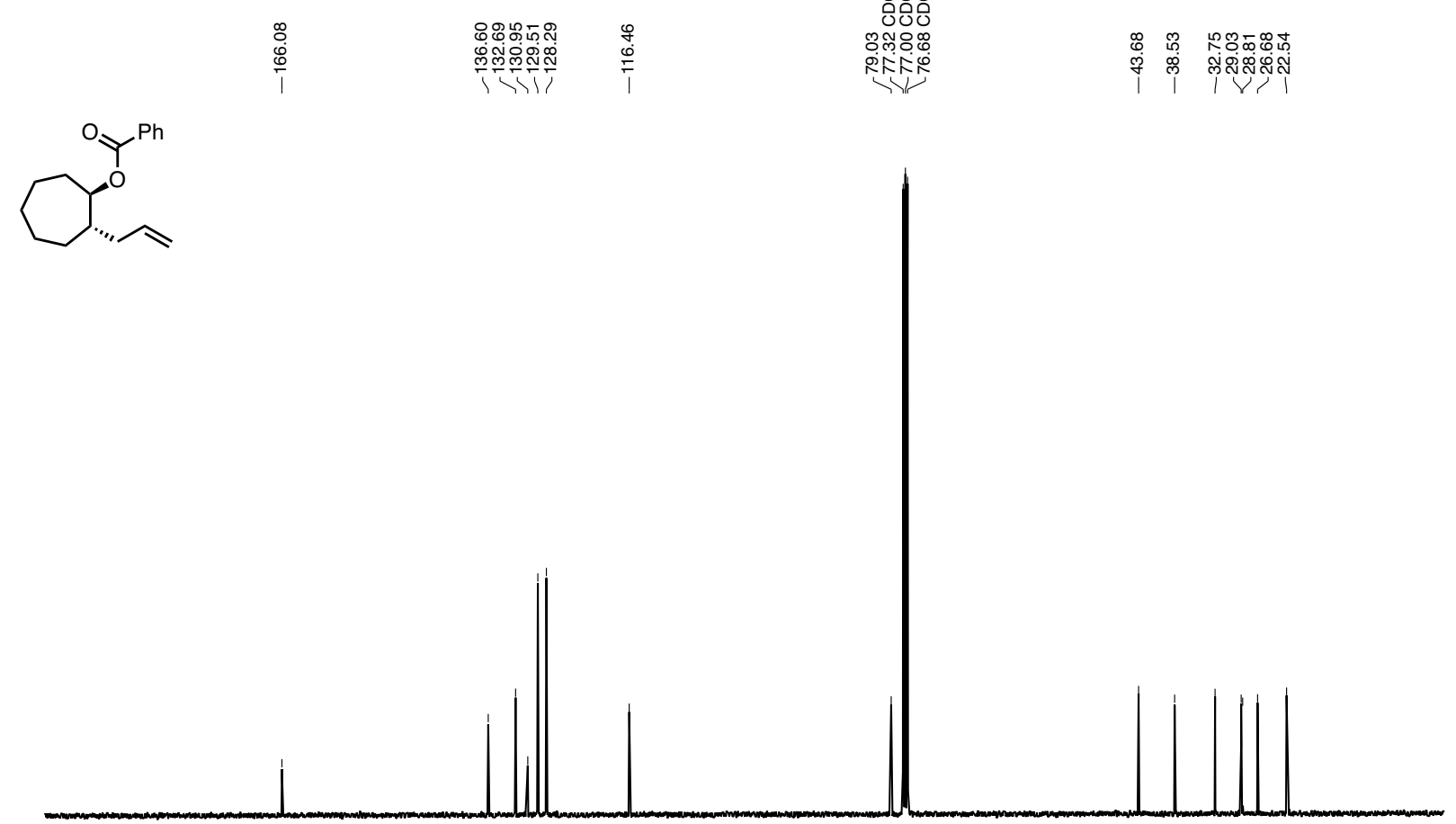

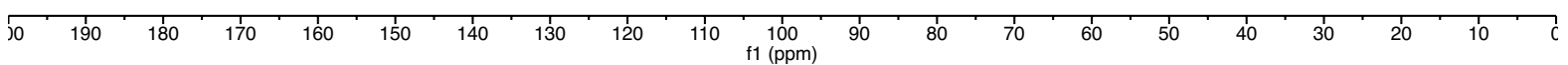


Tertiary alcohol $(+)-5$

\section{${ }^{1} \mathrm{H}$ NMR (400 MHz, $\left.\mathrm{CDCl}_{3}\right)$}

吕

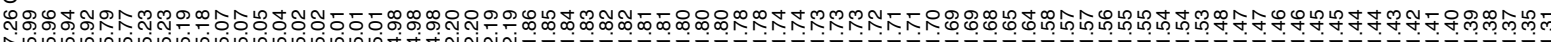
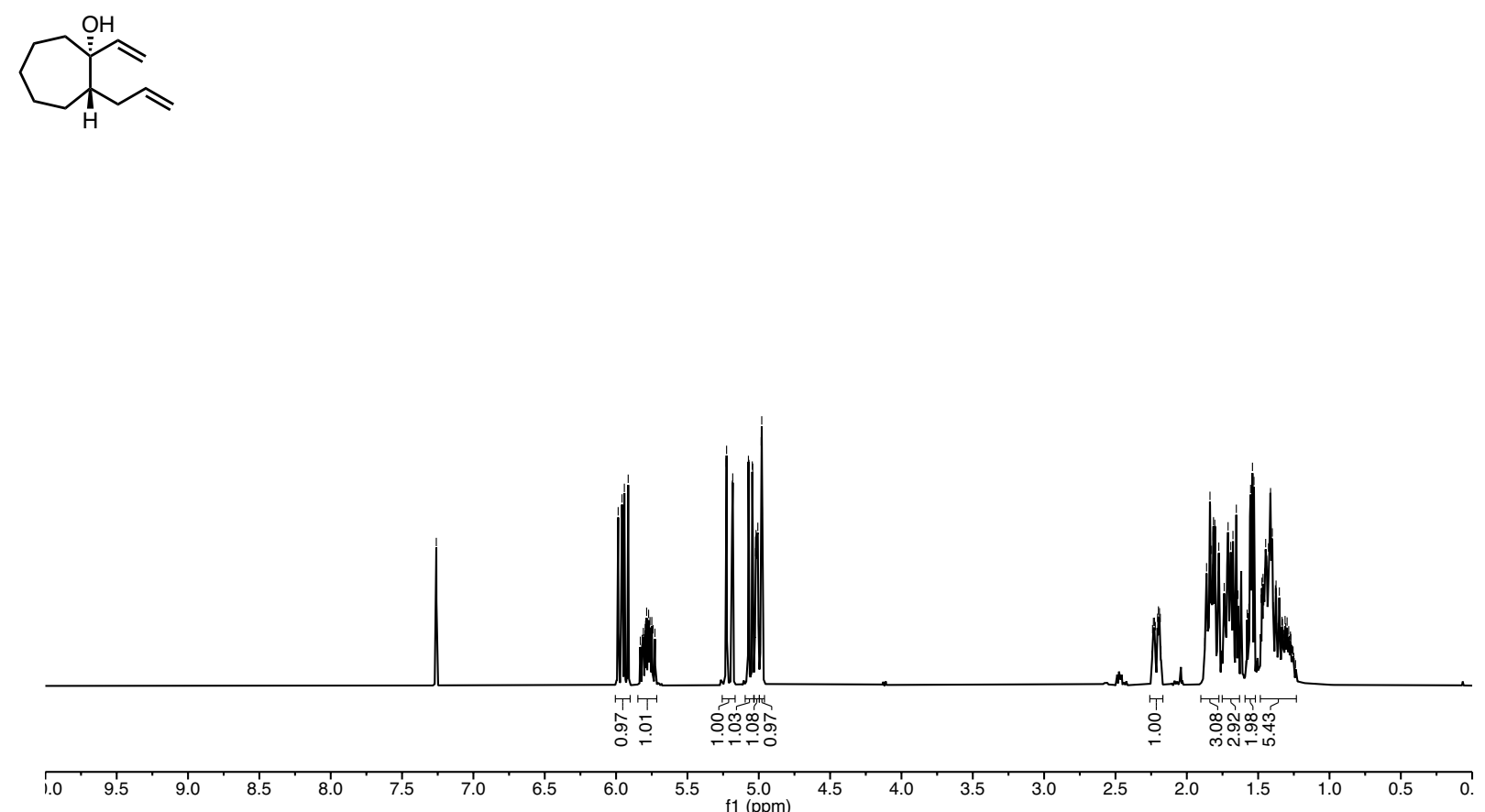

${ }^{13} \mathrm{C}$ NMR (101 MHz, $\left.\mathrm{CDCl}_{3}\right)$
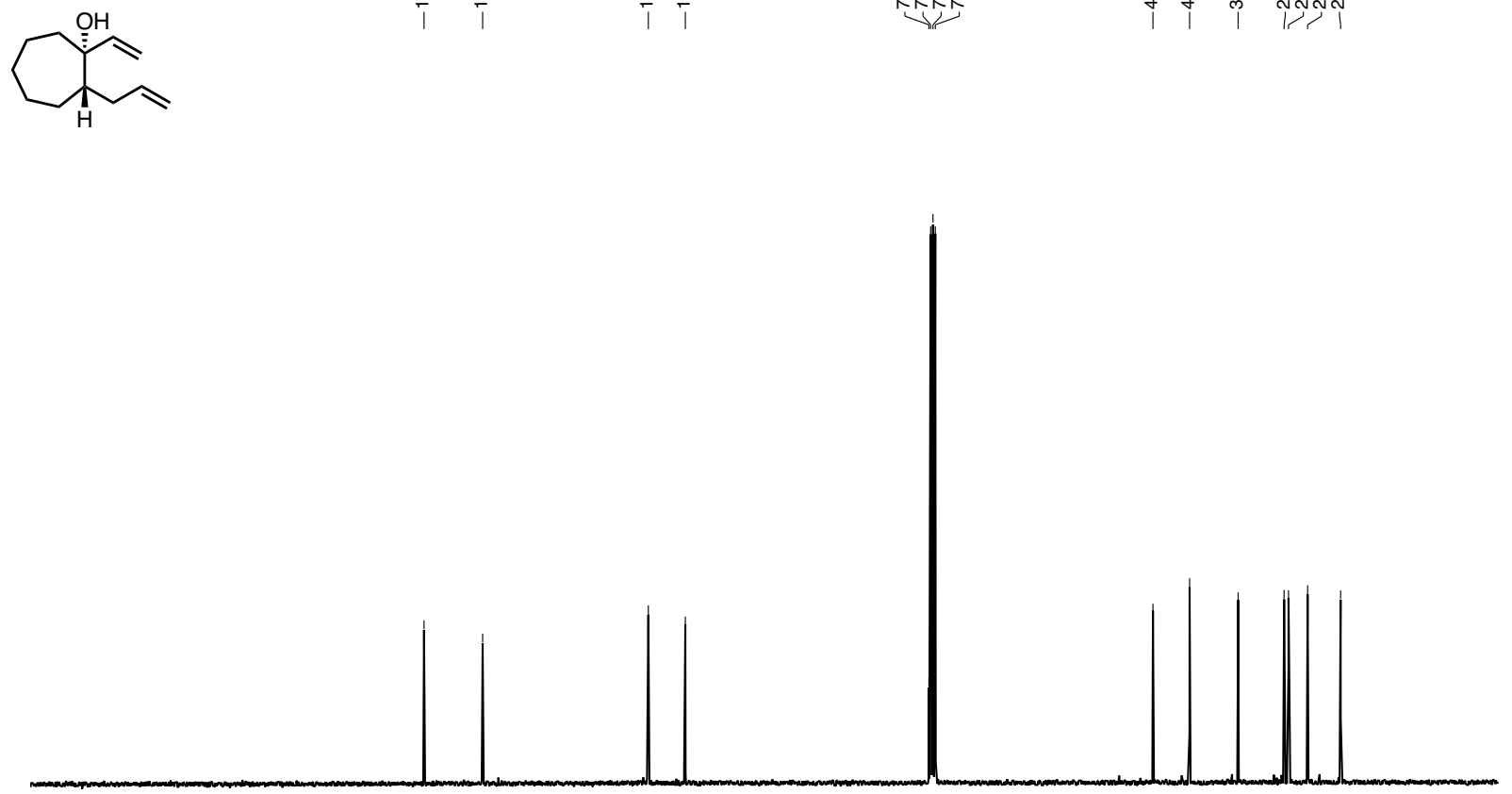

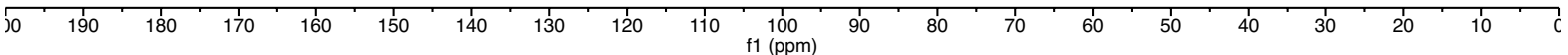


Cyclopentenone (-)-6

${ }^{1} \mathrm{H}$ NMR (400 MHz, $\left.\mathrm{CDCl}_{3}\right)$

递

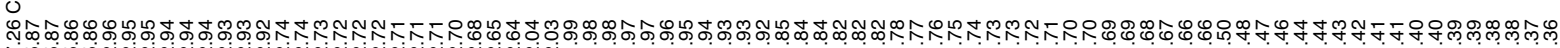

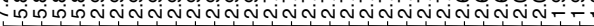<smiles>O=C1C=C2CCCCC[C@H]2C1</smiles>

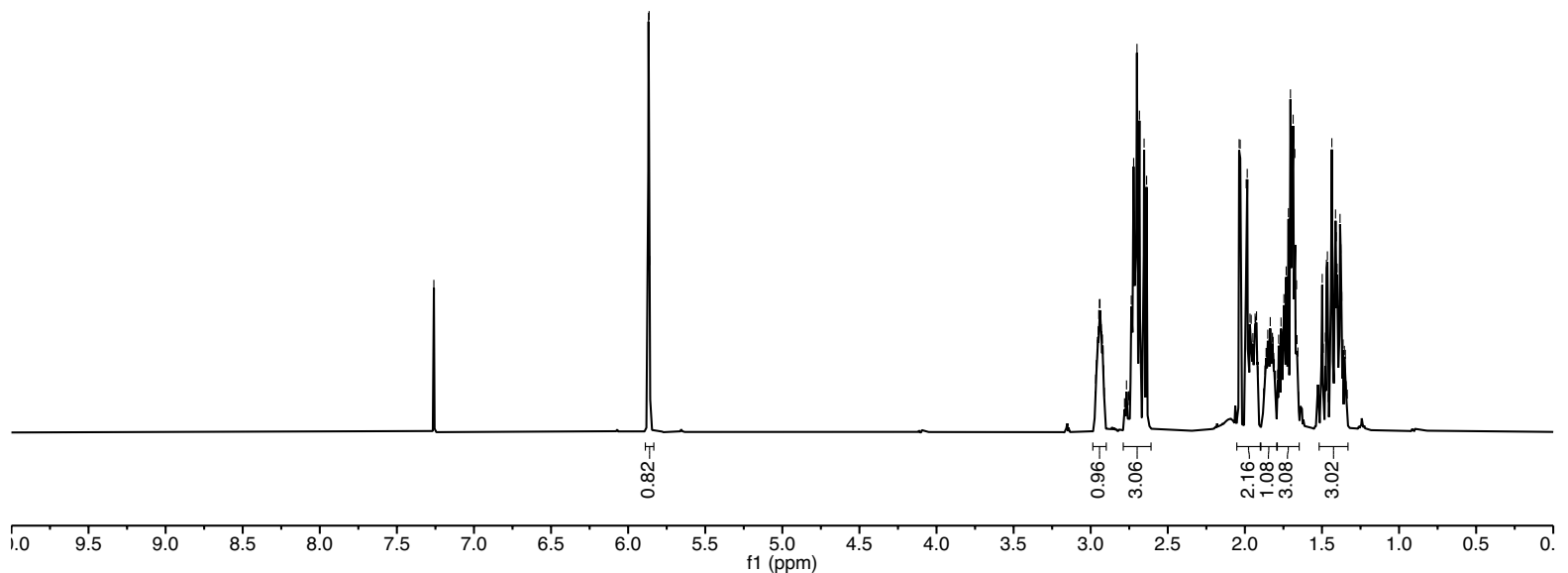

${ }^{13} \mathrm{C}$ NMR (101 MHz, $\left.\mathrm{CDCl}_{3}\right)$

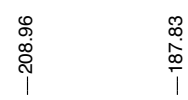

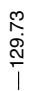
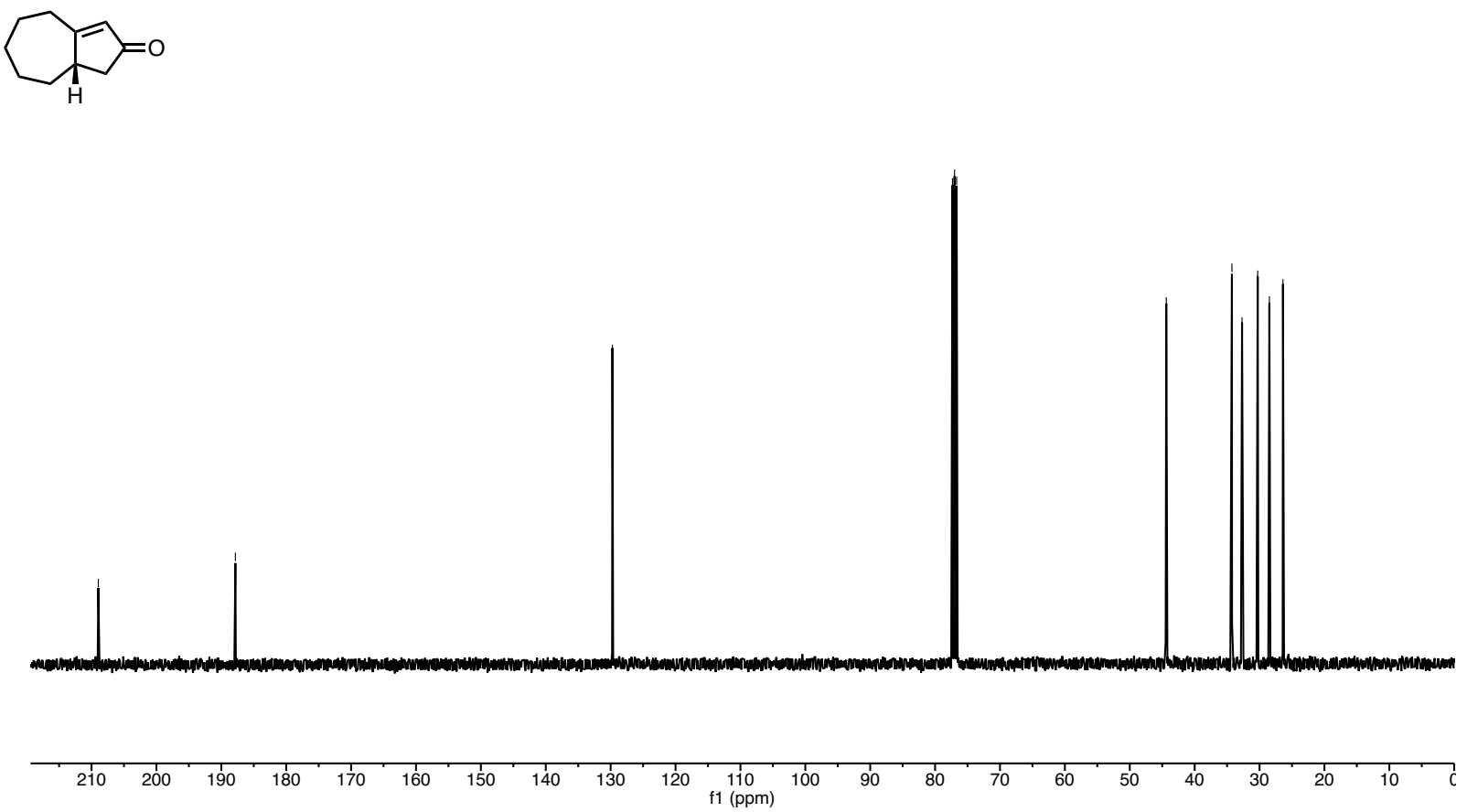
Monodeuterated ketone $(+)-d_{1}-\mathbf{8}$

${ }^{1} \mathrm{H}$ NMR (600 MHz, $\mathrm{CDCl}_{3}$ )

岂

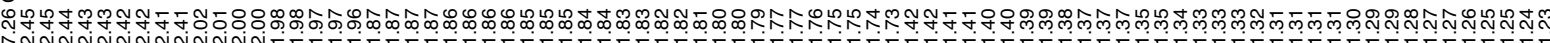<smiles>[2H][C@]12CCCCC[C@@H]1CC(=O)C2</smiles>

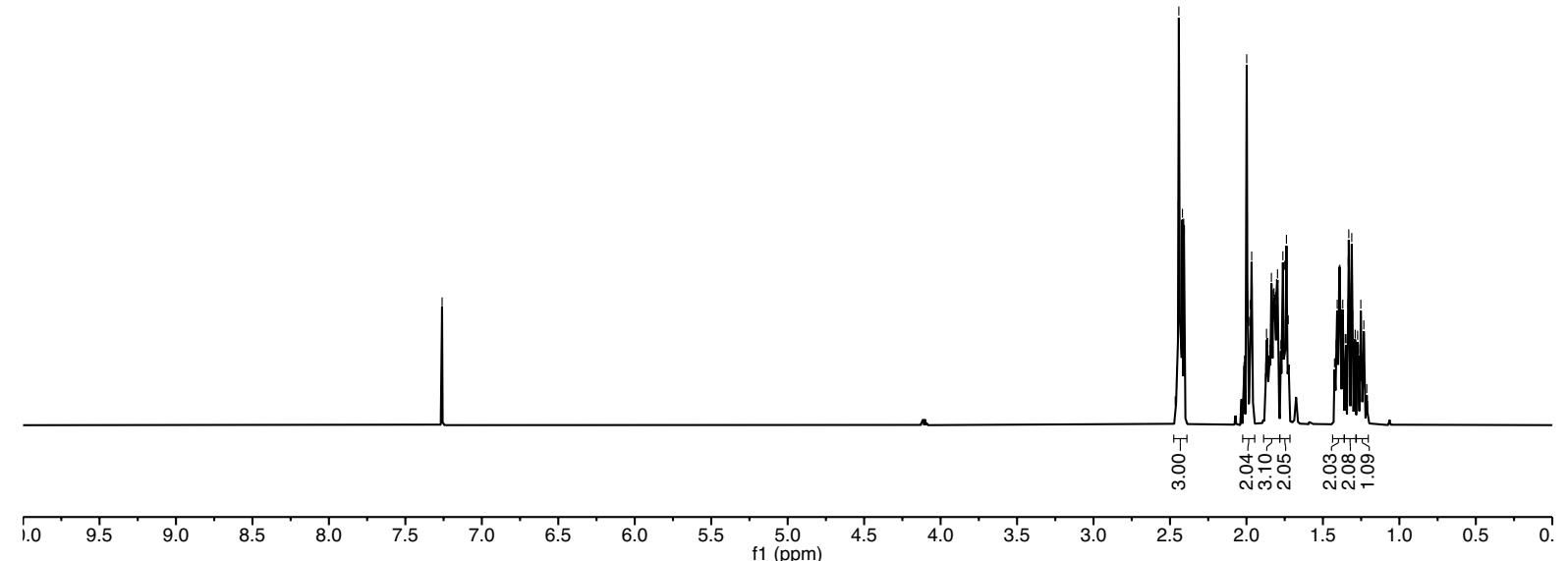

${ }^{2} \mathrm{H}$ NMR (92 MHz, $\mathrm{CDCl}_{3}$ )<smiles>[2H][C@]12CCCCC[C@@H]1CC(=O)C2</smiles>
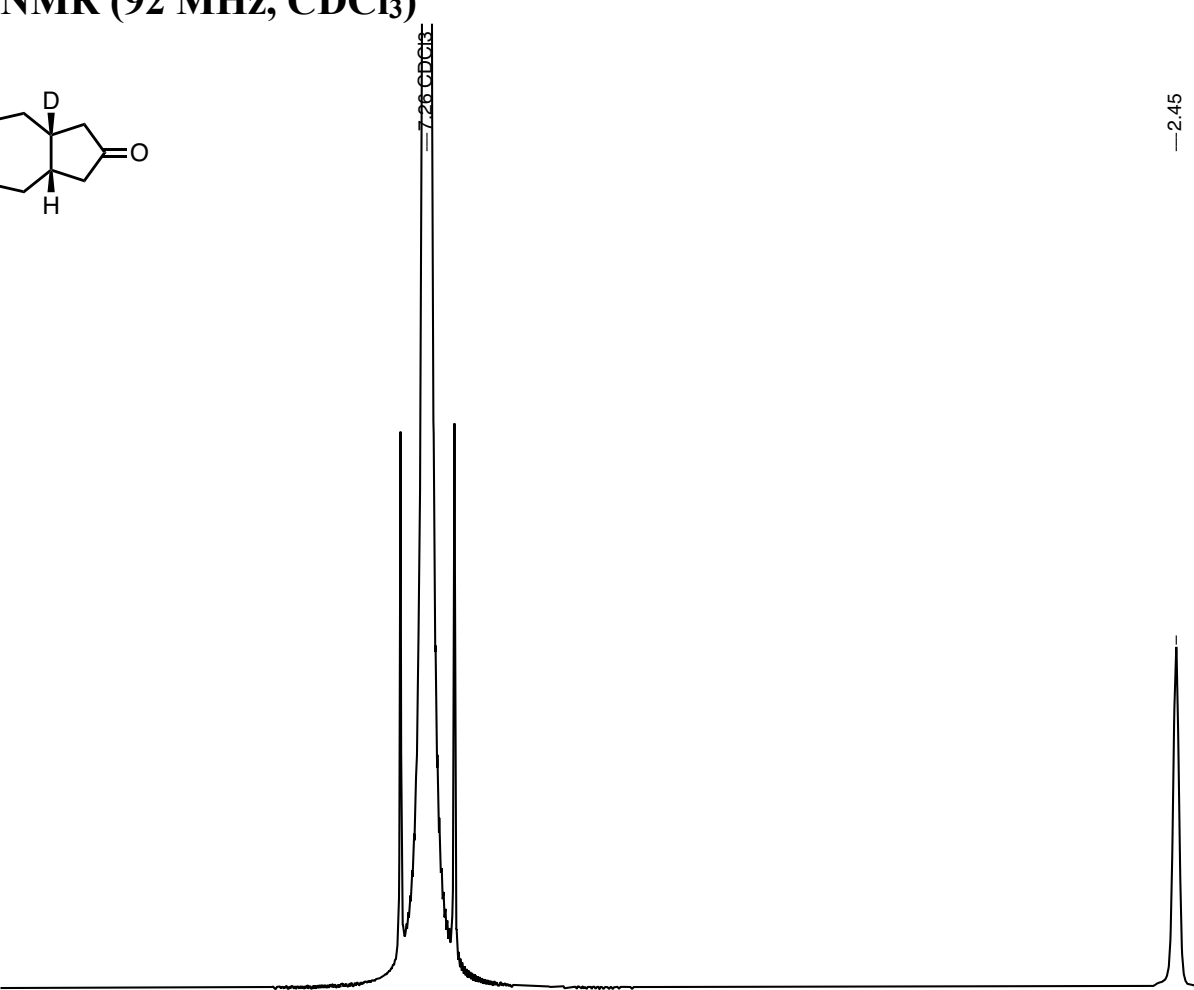

$\begin{array}{lll}9.5 & 9.0 & 8.5\end{array}$ 


\section{${ }^{13} \mathrm{C}$ NMR (151 MHz, CDCl 3$)$}

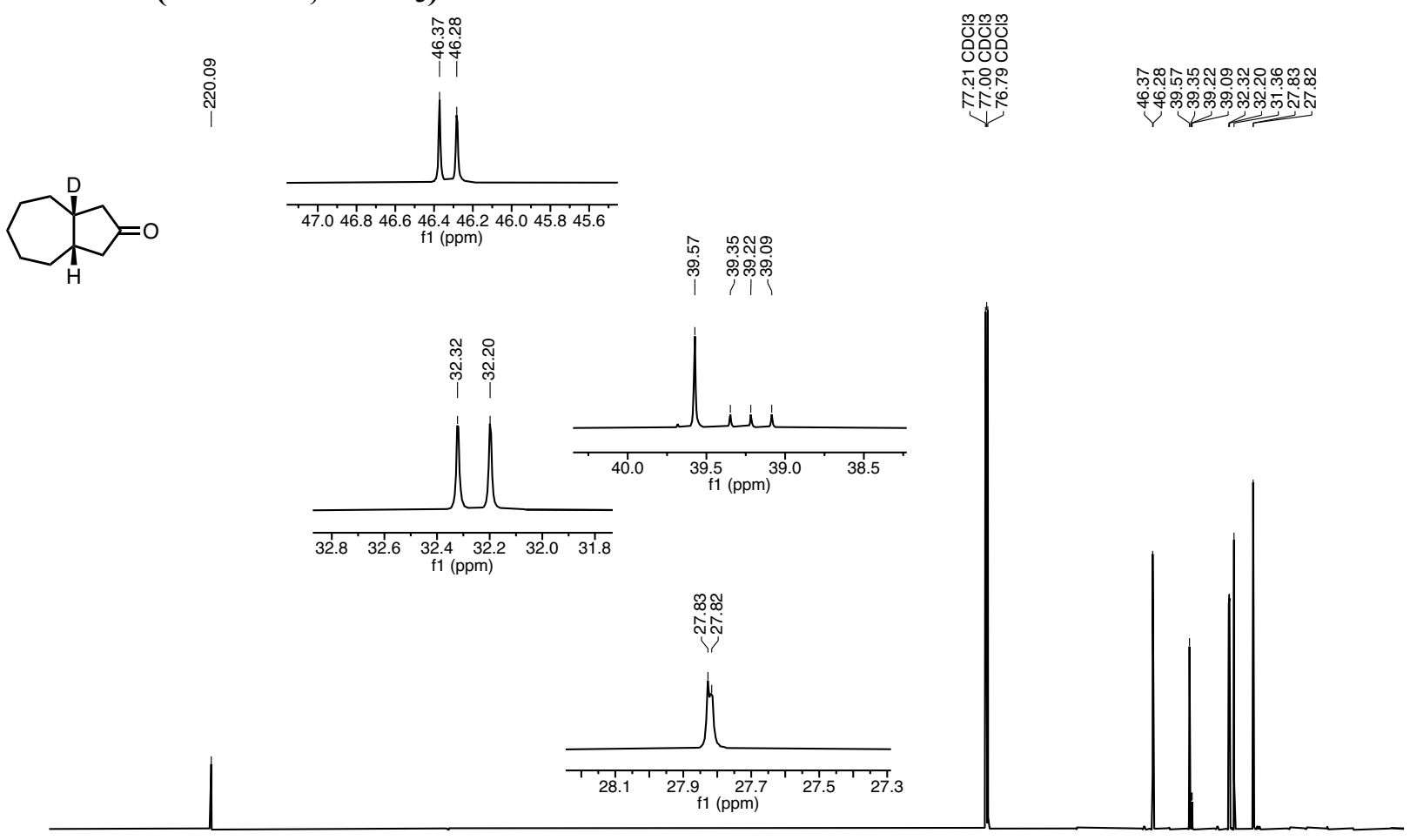

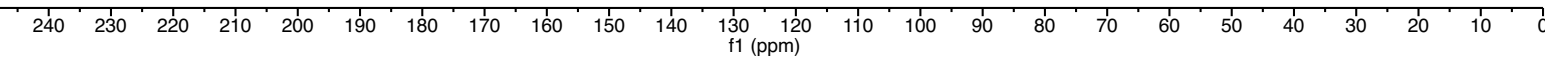


Monodeuterated cis-perhydroazulene $d_{1}-1$

${ }^{1} \mathrm{H}$ NMR (600 MHz, $\left.\mathrm{CDCl}_{3}\right)$

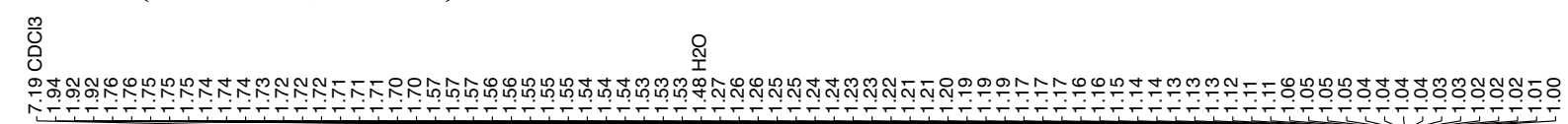<smiles>[2H][C@]12CCCC[C@H]1CCC2</smiles>

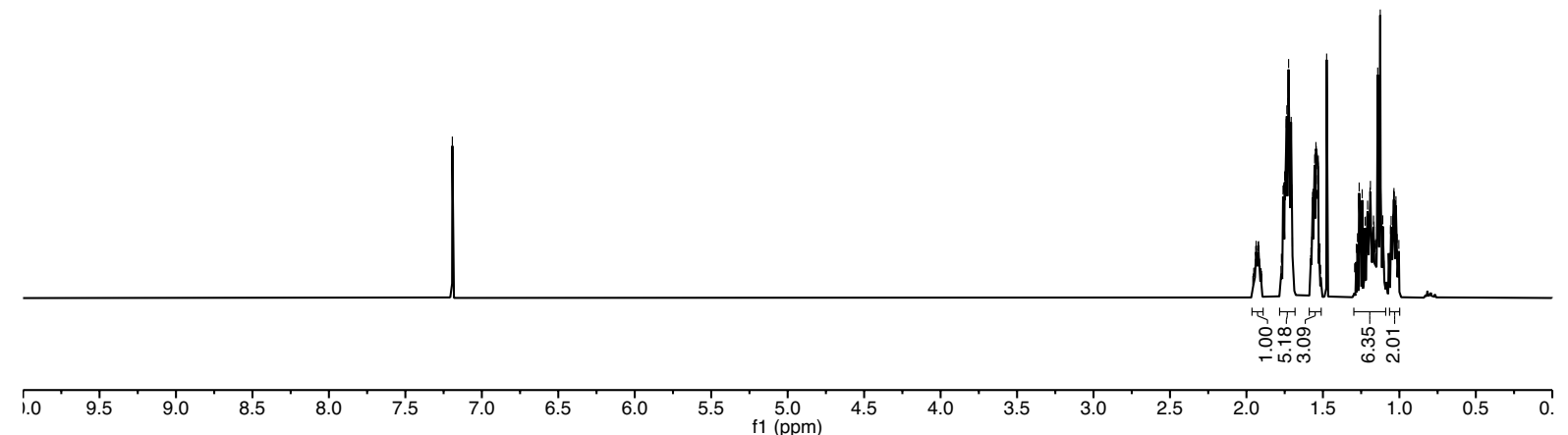

${ }^{2} \mathrm{H}$ NMR (92 MHz, $\left.\mathrm{CDCl}_{3}\right)$<smiles>[2H]C12CCCCCC1CCC2</smiles>

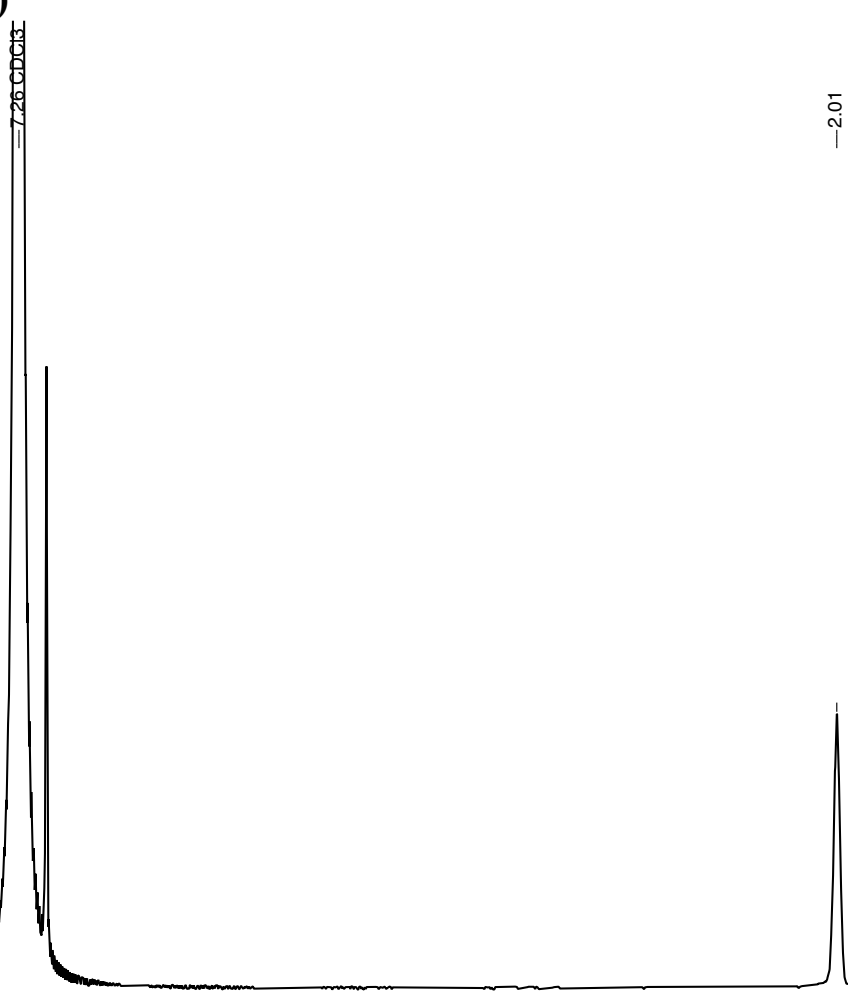

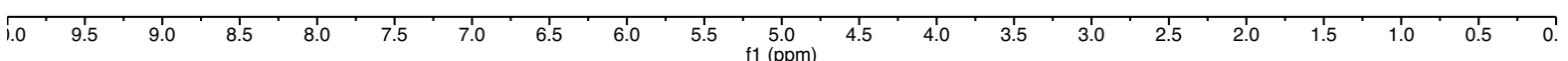


${ }^{13} \mathrm{C}$ NMR (151 MHz, $\left.\mathrm{CDCl}_{3}\right)$<smiles>C1CC[C@@H]2CCC[C]2C1</smiles>
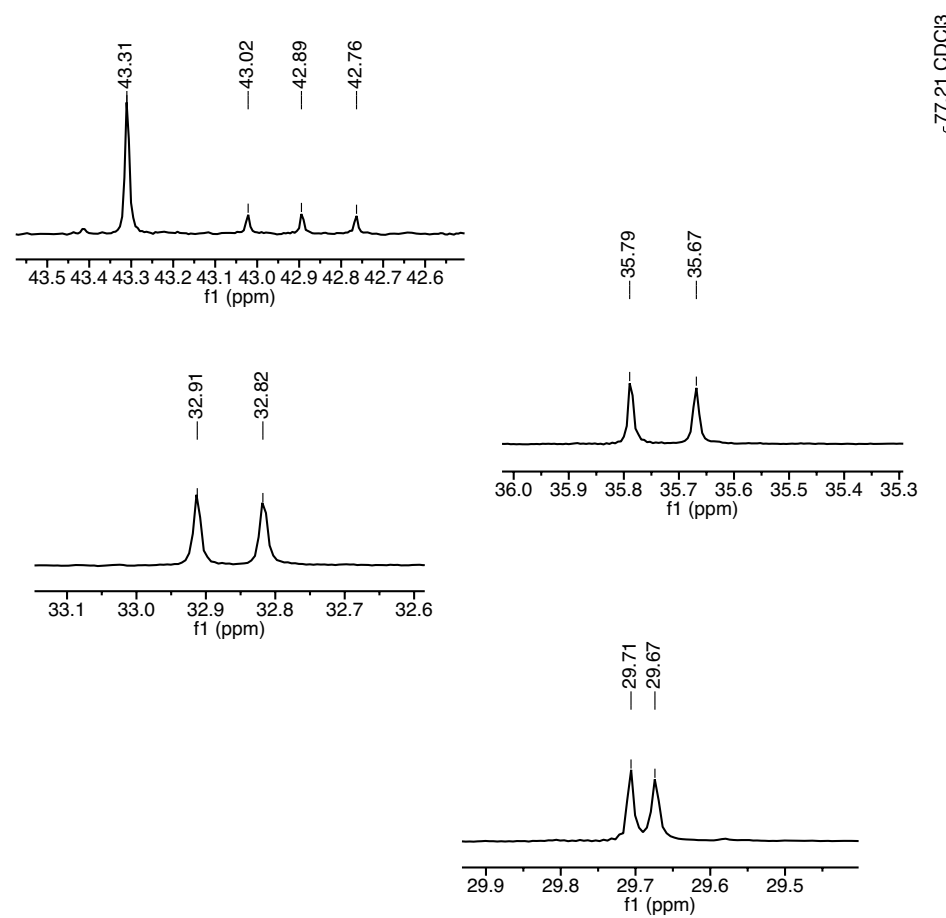

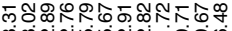

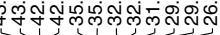
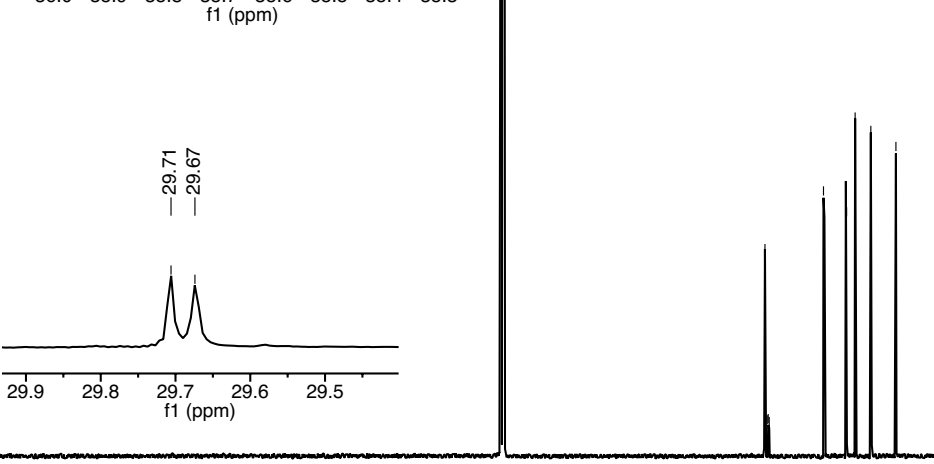

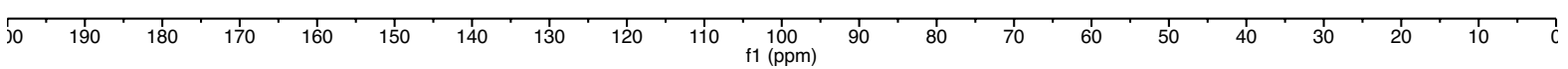


Imide (-)-S2

${ }^{1} \mathrm{H}$ NMR (400 MHz, $\mathrm{CDCl}_{3}$ )

$\frac{m}{0}$

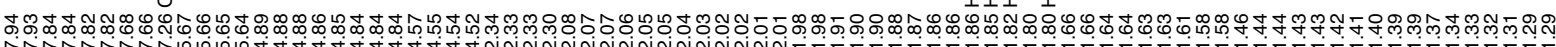<smiles>C=CC[C@H]1CCCCC[C@H]1N1C(=O)c2ccc(Br)cc2C1=O</smiles>

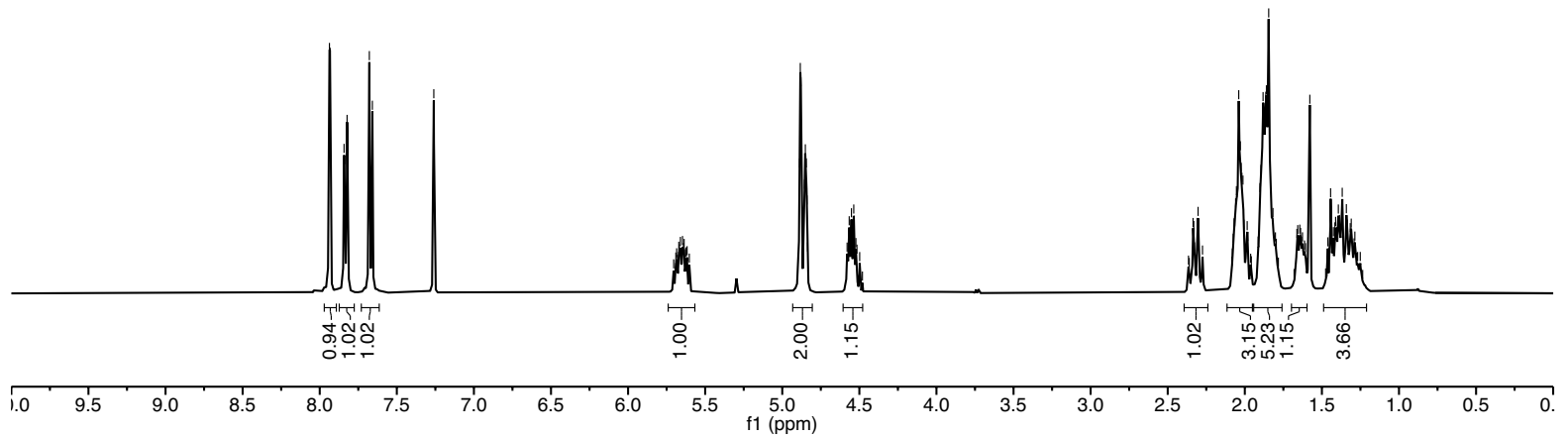

${ }^{13} \mathrm{C}$ NMR (101 MHz, $\left.\mathrm{CDCl}_{3}\right)$

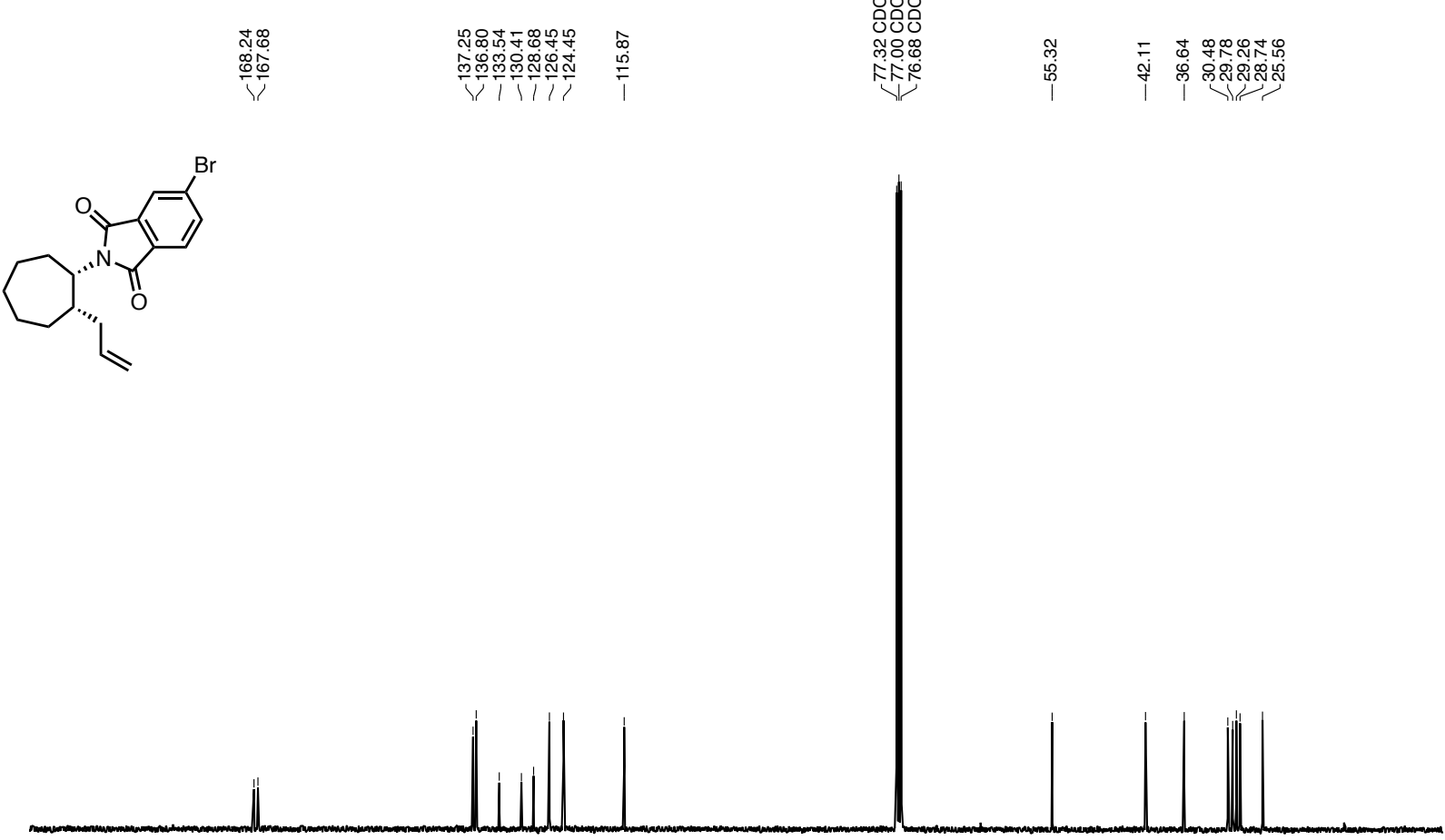

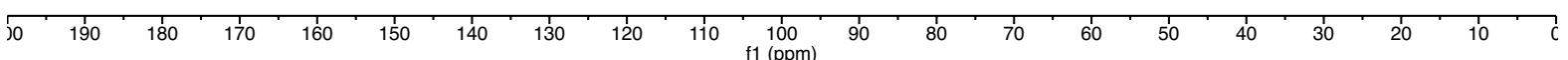


Sulfonamide (-)-9

\section{${ }^{1} \mathrm{H}$ NMR (400 $\mathrm{MHz}, \mathrm{CDCl}_{3}$ )}

遌

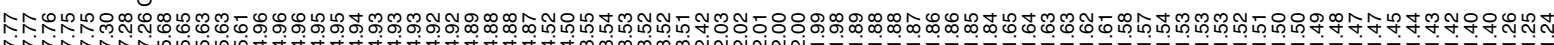<smiles>C=CC[C@H]1CCCCC[C@H]1NS(=O)(=O)c1ccc([N+](=O)[O-])cc1</smiles>

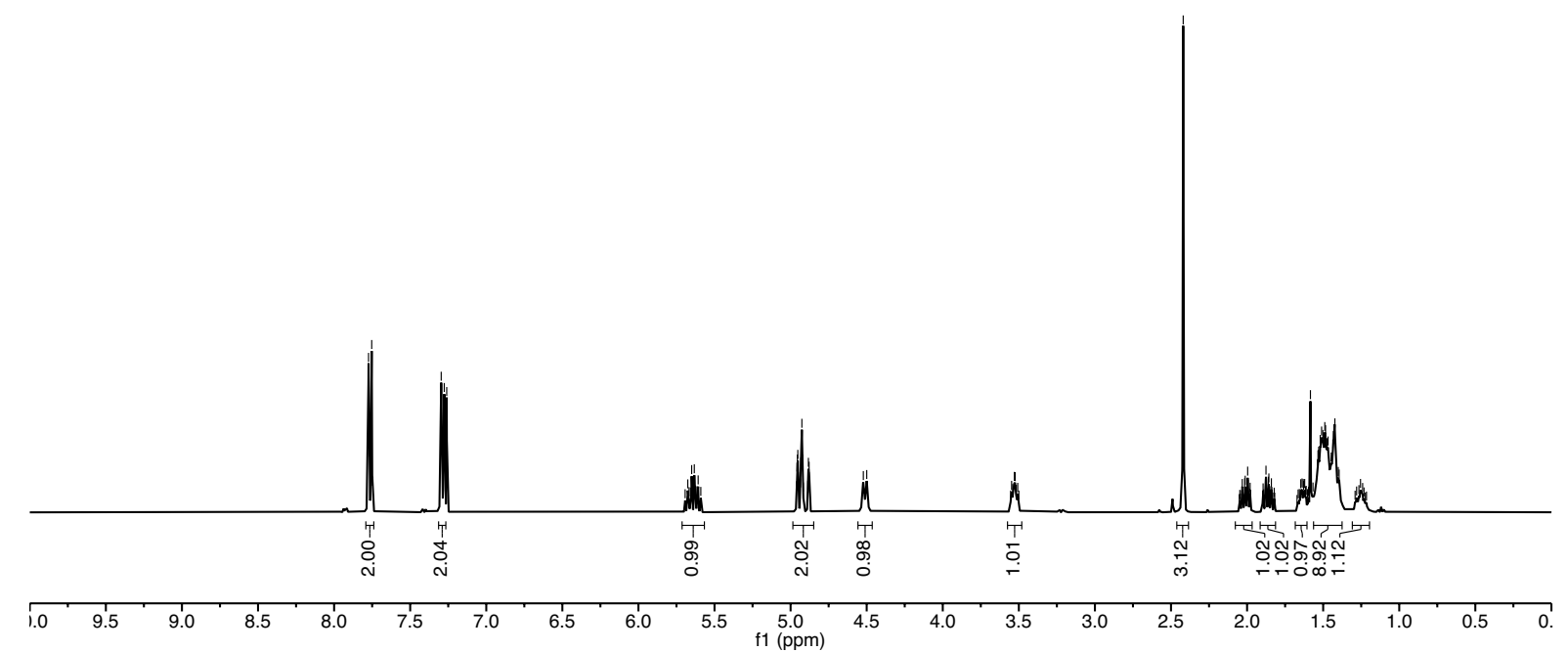

${ }^{13} \mathrm{C}$ NMR (101 MHz, $\left.\mathrm{CDCl}_{3}\right)$<smiles>C=CC[C@H]1CCCCC[C@H]1NS(=O)(=O)c1ccc(C)cc1</smiles>
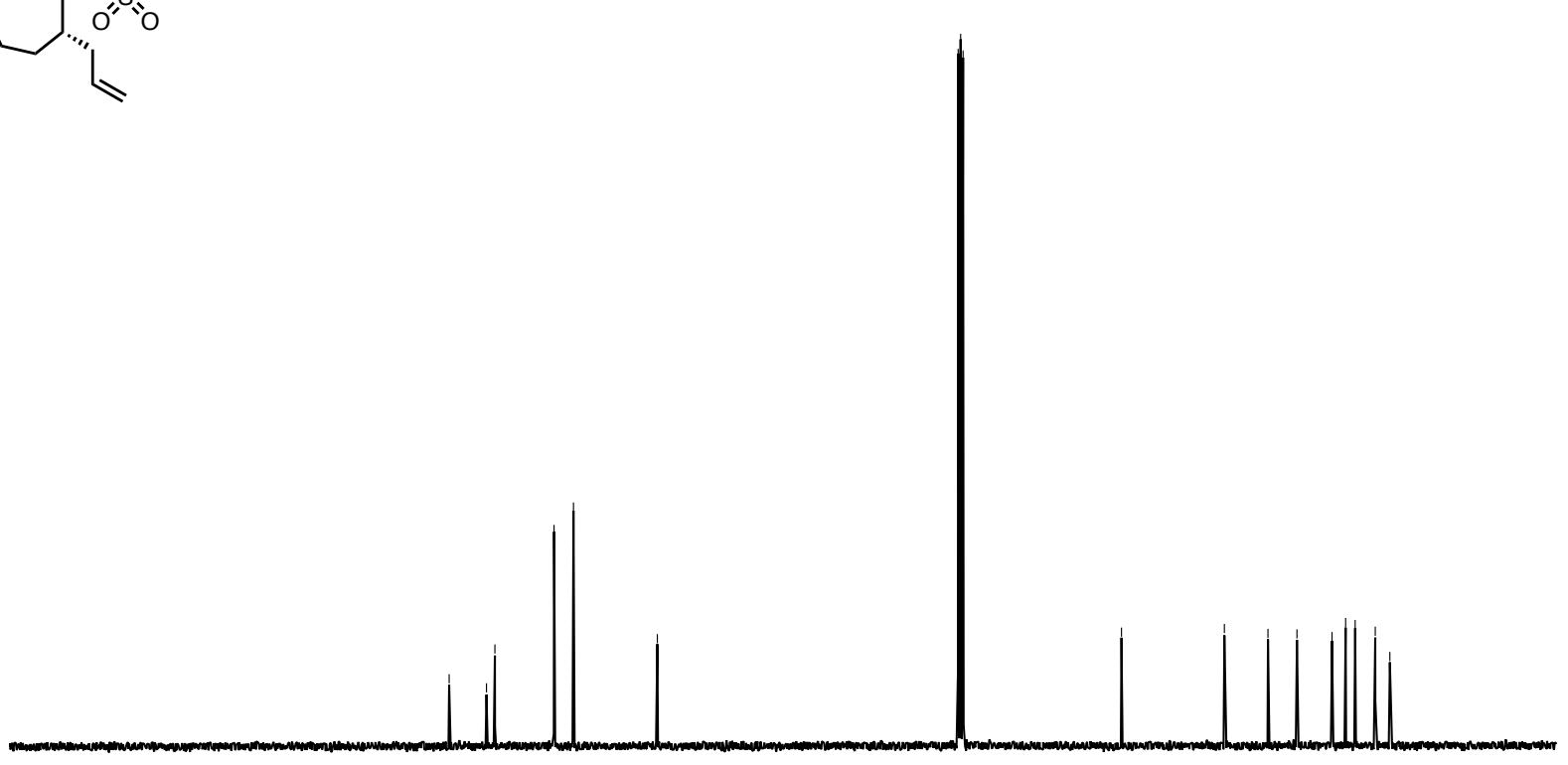

\begin{tabular}{llllll}
\hline 0 & 190 & 180 & 170 & 160 & 150
\end{tabular} 100
$\mathrm{f} 1(\mathrm{ppm})$ 


\section{X-ray Crystallographic Analysis}<smiles>C=CC[C@H]1CCCCC[C@H]1NS(=O)(=O)c1ccc(C)cc1</smiles>

$(-)-9$

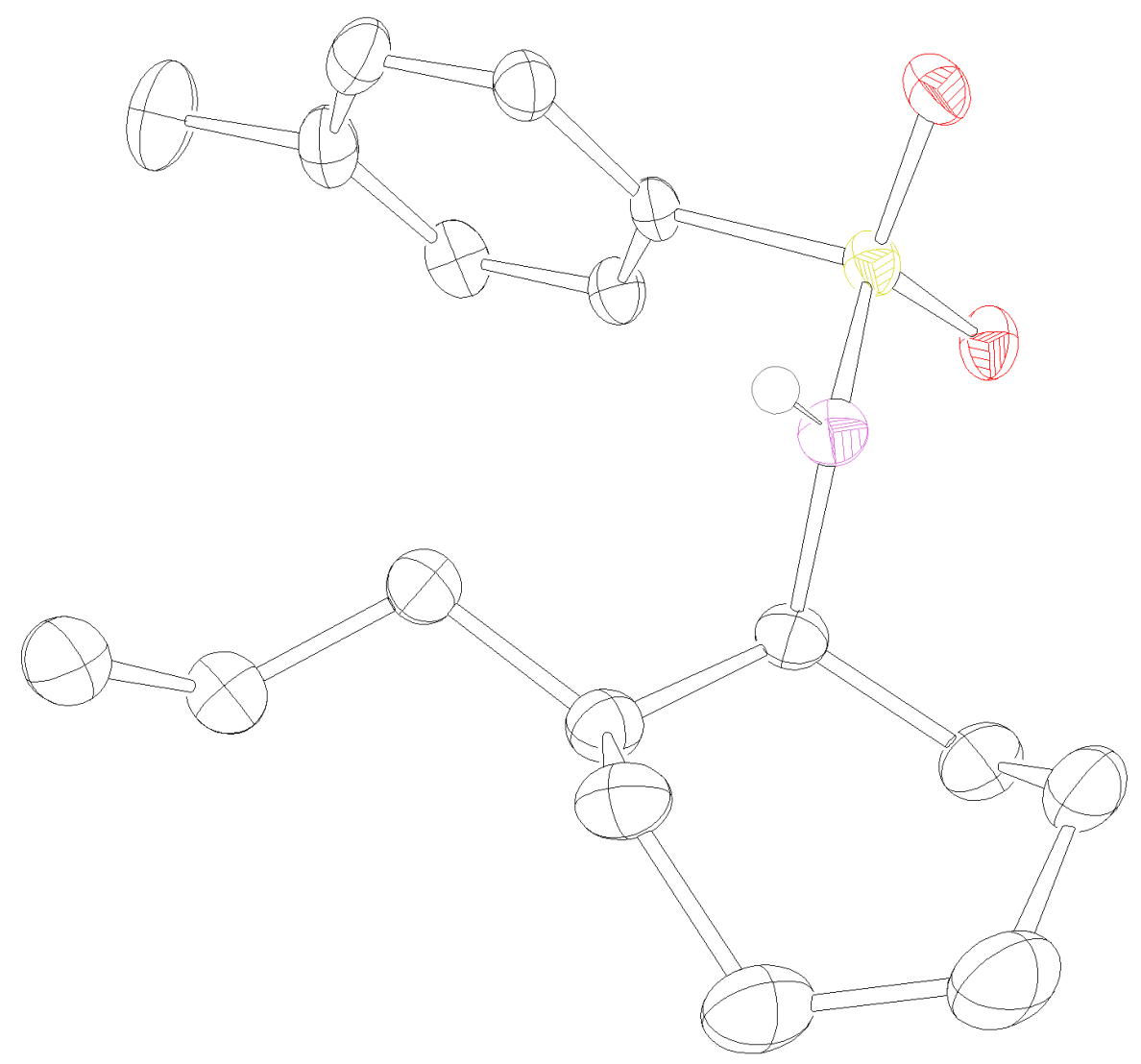

Figure S2. Thermal ellipsoid plot of (-)-9 in $P 2{ }_{1} 2{ }_{1} 2$. Thermal ellipsoids are set at the 50\% probability level. $\mathrm{C}-\mathrm{H}$ hydrogen atoms and second molecule in asymmetric unit omitted for clarity. 


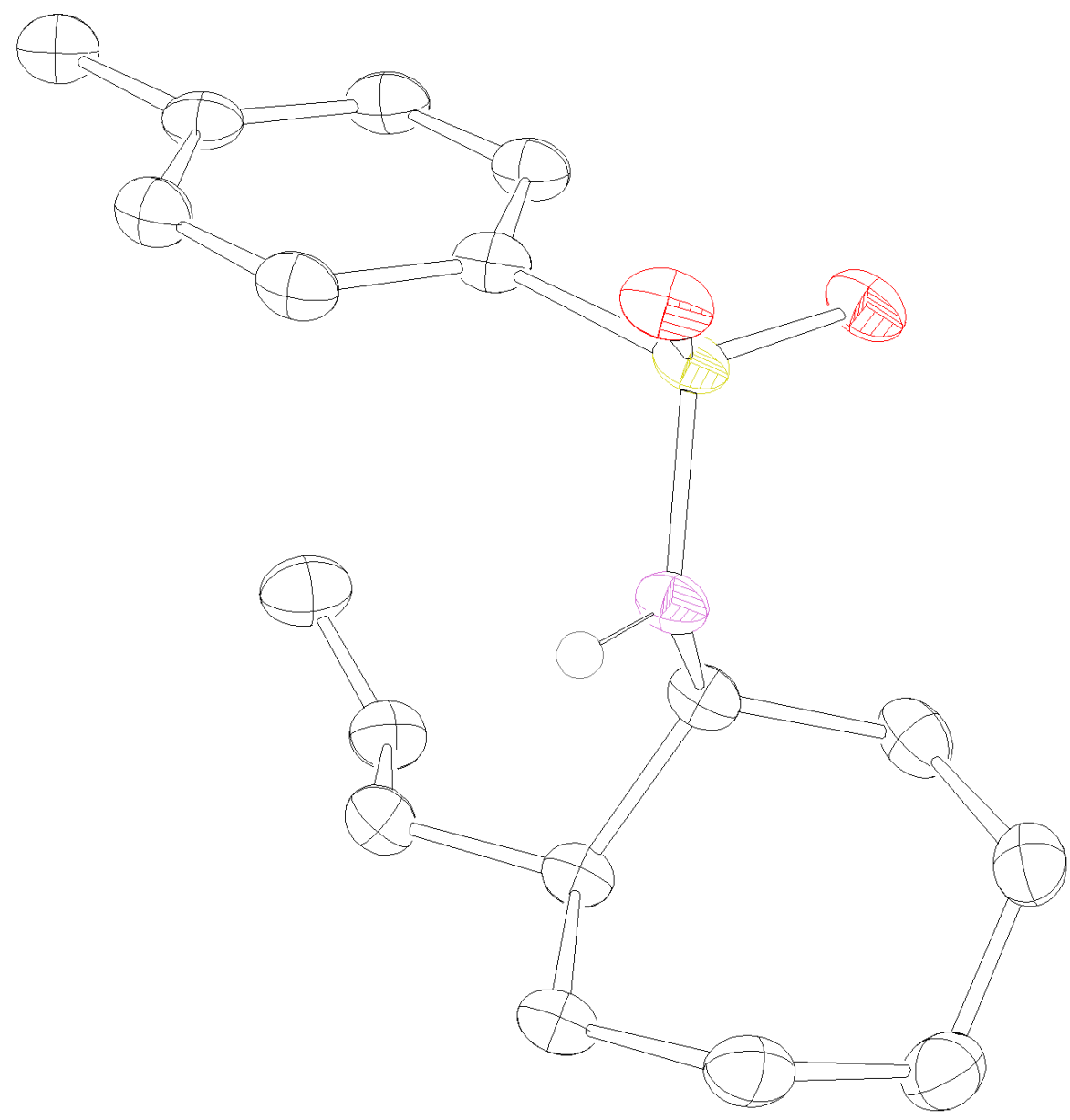

Figure S3. Thermal ellipsoid plot of (-)-9 in $P 4_{3} 2_{1} 2$. Thermal ellipsoids are set at the $50 \%$ probability level. $\mathrm{C}-\mathrm{H}$ hydrogen atoms omitted for clarity.

\section{Crystallization method}

Slow evaporation (solvent: methanol/ $\mathrm{H}_{2} \mathrm{O}$ )

\section{Experimental}

During crystallization of (-)-9 two different kind of crystal shapes (needles and blocks) could be found. Diffraction data for both were collected at low temperatures $(100 \mathrm{~K})$ using $\varphi$ - and $\omega$-scans on a BRUKER D8 Venture system equipped with dual $\mathrm{I} \mu \mathrm{S}$ microfocus sources, a PHOTON100 detector and an OXFORD CRYOSYSTEMS 700 low temperature system. $\mathrm{Cu}-\mathrm{K}_{\alpha}$ radiation with wavelength $1.54178 \AA, \mathrm{Mo}_{\alpha} \mathrm{K}_{\alpha}$ radiation with wavelength $0.71073 \AA$ and a collimating Quazar multilayer mirror were used. Semi-empirical absorption correction from equivalents was applied using SADABS$2016 / 2^{5}$ and the structures were solved by direct methods using SHELXT2014/5 . Refinement was performed against $F^{2}$ on all data by full-matrix least squares using SHELXL2018/3. ${ }^{7}$ All nonhydrogen atoms were refined anisotropically, $\mathrm{N}-\mathrm{H}$ hydrogen atoms were located in the Fourier 
synthesis and set to ideal distances, and $\mathrm{C}-\mathrm{H}$ hydrogen atoms were positioned at geometrically calculated positions and refined using a riding model. The isotropic displacement parameters of all hydrogen atoms were fixed to $1.2 \mathrm{x}$ or $1.5 \mathrm{x}\left(\mathrm{CH}_{3}\right)$ the $\mathrm{U}_{\text {eq }}$ value of the atoms they are linked to.

The crystallographic data has been deposited with the Cambridge Crystallographic Data Centre as CCDC No. 2041505-2041506 and can be obtained free of charge. ${ }^{8}$

\section{$(-)-9$ in $P 2_{1} 2_{1} 2$}

The structure was solved in the orthorhombic space group $P 2{ }_{1} 2{ }_{1} 2$. The asymmetric unit contains two molecules of (-)-9 connected via hydrogen bonds. One of the cycloheptyl groups was found to be disordered over two positions. The disorder was refined using same distance restraints on 1,2distances, similarity restraints on the anisotropic displacement parameters ${ }^{9}$ and advanced rigid bond restraints. ${ }^{10}$ Atoms close to each other were set to the same anisotropic displacement parameters to allow for full anisotropic refinement of the model. The disorder ratio was allowed to refine freely and converged to $0.797(8)$. Anomalous dispersion was used to determine the absolute structure and a Parsons parameter of $0.009(24)$ confirmed the assignment. ${ }^{11}$ 
Table S1. Crystal data and structure refinement for (-)-9 in $\boldsymbol{P 2} \mathbf{2}_{1} \mathbf{2}$.

\section{CCDC No}

Empirical formula

Formula weight

Temperature

Wavelength

Crystal system

Space group

Unit cell dimensions

Volume

Z

Density (calculated)

Absorption coefficient

$F(000)$

Crystal size

Theta range for data collection

Index ranges

Reflections collected

Independent reflections

Completeness to theta $=25.242^{\circ}$

Absorption correction

Refinement method

Data / restraints / parameters

Goodness-of-fit on $F^{2}$

Final $R$ indices $[\mathrm{I}>2 \sigma(\mathrm{I})]$

$\mathrm{R}$ indices (all data)

Absolute structure parameter

Largest diff. peak and hole
2041505

$\mathrm{C}_{17} \mathrm{H}_{25} \mathrm{~N} \mathrm{O}_{2} \mathrm{~S}$

307.44

$100(2) \mathrm{K}$

$0.71073 \AA$

Orthorhombic

$P 21212$

$\mathrm{a}=15.7391(8) \AA \quad \alpha=90^{\circ}$.

$\mathrm{b}=21.4452(11) \AA \quad \beta=90^{\circ}$.

$\mathrm{c}=9.8436(5) \AA \quad \gamma=90^{\circ}$.

$3322.5(3) \AA^{3}$

8

$1.229 \mathrm{Mg} / \mathrm{m}^{3}$

$0.199 \mathrm{~mm}^{-1}$

1328

$0.729 \times 0.112 \times 0.099 \mathrm{~mm}^{3}$

1.605 to $27.877^{\circ}$.

$-20 \leq \mathrm{h} \leq 20,-28 \leq \mathrm{k} \leq 28,-12 \leq 1 \leq 12$

87189

$7930[\mathrm{R}($ int $)=0.0830]$

$100.0 \%$

Semi-empirical from equivalents

Full-matrix least-squares on $F^{2}$

7930 / 242 / 424

1.037

$\mathrm{R} 1=0.0421, \mathrm{wR} 2=0.0905$

$\mathrm{R} 1=0.0593, \mathrm{wR} 2=0.0977$

$0.01(2)$

0.251 and -0.389 e. $\AA^{-3}$ 
Table S2. Atomic coordinates (x 104) and equivalent isotropic displacement parameters $\left(\AA^{2} \times 10^{3}\right)$ for (-)-9 in $\boldsymbol{P 2}_{1} \mathbf{2}_{1} \mathbf{2}$. $\mathrm{U}(\mathrm{eq})$ is defined as one third of the trace of the orthogonalized $\mathrm{U}^{\mathrm{ij}}$ tensor.

\begin{tabular}{|c|c|c|c|c|}
\hline & $\mathrm{x}$ & $\mathrm{y}$ & z & $\mathrm{U}(\mathrm{eq})$ \\
\hline$S(1)$ & $2634(1)$ & $7758(1)$ & $6707(1)$ & $19(1)$ \\
\hline $\mathrm{O}(1)$ & 2074(1) & $8224(1)$ & $7240(2)$ & $24(1)$ \\
\hline $\mathrm{O}(2)$ & $2626(1)$ & $7138(1)$ & $7273(2)$ & $23(1)$ \\
\hline $\mathrm{N}(1)$ & 3593(2) & $8006(1)$ & $6888(3)$ & $21(1)$ \\
\hline $\mathrm{C}(1)$ & $5624(2)$ & $8192(2)$ & 2639(4) & $41(1)$ \\
\hline $\mathrm{C}(2)$ & $5044(2)$ & $8524(2)$ & $3233(4)$ & $35(1)$ \\
\hline $\mathrm{C}(3)$ & $4484(2)$ & $8318(2)$ & $4369(3)$ & $28(1)$ \\
\hline$C(4)$ & $4607(2)$ & $8695(2)$ & $5684(3)$ & $26(1)$ \\
\hline $\mathrm{C}(5)$ & $5452(2)$ & $8537(2)$ & $6385(4)$ & $31(1)$ \\
\hline $\mathrm{C}(6)$ & $5758(2)$ & $9044(2)$ & $7369(4)$ & $42(1)$ \\
\hline $\mathrm{C}(7)$ & $5411(3)$ & 9011(2) & $8815(4)$ & $49(1)$ \\
\hline$C(8)$ & $4499(2)$ & $8773(2)$ & 8961(4) & $38(1)$ \\
\hline $\mathrm{C}(9)$ & $3863(2)$ & $9028(2)$ & 7924(4) & $35(1)$ \\
\hline$C(10)$ & $3818(2)$ & $8662(1)$ & $6590(3)$ & $23(1)$ \\
\hline $\mathrm{C}(11)$ & $2422(2)$ & $7686(1)$ & 4951(3) & $18(1)$ \\
\hline$C(12)$ & $2719(2)$ & $7169(1)$ & $4248(3)$ & $24(1)$ \\
\hline $\mathrm{C}(13)$ & $2596(2)$ & $7133(1)$ & 2862(3) & $29(1)$ \\
\hline$C(14)$ & $2188(2)$ & $7607(2)$ & $2155(3)$ & $31(1)$ \\
\hline$C(15)$ & 1890(2) & $8117(2)$ & $2876(3)$ & $27(1)$ \\
\hline$C(16)$ & $1996(2)$ & $8159(1)$ & $4267(3)$ & $22(1)$ \\
\hline $\mathrm{C}(17)$ & 2061(3) & $7568(2)$ & $634(4)$ & $50(1)$ \\
\hline$S(21)$ & $4660(1)$ & $6301(1)$ & $7121(1)$ & $17(1)$ \\
\hline $\mathrm{O}(21)$ & $5239(1)$ & $5840(1)$ & $6628(2)$ & $22(1)$ \\
\hline $\mathrm{O}(22)$ & 4693(1) & $6924(1)$ & $6569(2)$ & $20(1)$ \\
\hline $\mathrm{N}(21)$ & $3704(2)$ & $6059(1)$ & $6854(2)$ & $18(1)$ \\
\hline$C(21)$ & $3057(2)$ & $5101(2)$ & $2278(4)$ & $36(1)$ \\
\hline$C(22)$ & $3247(2)$ & $4859(1)$ & $3460(3)$ & $25(1)$ \\
\hline $\mathrm{C}(23)$ & $3547(2)$ & $5218(1)$ & $4665(3)$ & $23(1)$ \\
\hline C(24) & 2983(2) & $5135(1)$ & $5927(3)$ & $19(1)$ \\
\hline$C(25)$ & $2075(8)$ & $5390(11)$ & $5693(13)$ & $22(2)$ \\
\hline$C(26)$ & 1384(7) & $5004(5)$ & $6388(7)$ & $27(2)$ \\
\hline $\mathrm{C}(27)$ & 1374(4) & $5031(3)$ & 7937(7) & $28(1)$ \\
\hline$C(28)$ & $2212(3)$ & $4920(2)$ & $8668(4)$ & $29(1)$ \\
\hline $\mathrm{C}(29)$ & $2860(4)$ & $5449(3)$ & $8476(6)$ & $25(1)$ \\
\hline $\mathrm{C}(30)$ & $3411(4)$ & $5432(3)$ & 7192(7) & 19(1) \\
\hline
\end{tabular}




$\begin{array}{lllll}\mathrm{C}(25 \mathrm{~A}) & 2100(30) & 5340(50) & 5800(50) & 22(2) \\ \mathrm{C}(26 \mathrm{~A}) & 1470(30) & 4990(20) & 6710(30) & 35(6) \\ \mathrm{C}(27 \mathrm{~A}) & 1483(17) & 5184(17) & 8190(30) & 49(7) \\ \mathrm{C}(28 \mathrm{~A}) & 2224(10) & 5558(9) & 8706(16) & 39(4) \\ \mathrm{C}(29 \mathrm{~A}) & 3105(13) & 5266(12) & 8510(30) & 29(5) \\ \mathrm{C}(30 \mathrm{~A}) & 3515(16) & 5362(13) & 7110(30) & 19(1) \\ \mathrm{C}(31) & 4799(2) & 6359(1) & 8889(3) & 17(1) \\ \mathrm{C}(32) & 5196(2) & 5885(1) & 9602(3) & 25(1) \\ \mathrm{C}(33) & 5246(2) & 5916(2) & 10999(3) & 30(1) \\ \mathrm{C}(34) & 4922(2) & 6421(1) & 11711(3) & 22(1) \\ \mathrm{C}(35) & 4534(2) & 6897(1) & 10976(3) & 24(1) \\ \mathrm{C}(36) & 4467(2) & 6867(1) & 9577(3) & 22(1) \\ \mathrm{C}(37) & 4997(2) & 6457(2) & 13233(3) & 28(1)\end{array}$


Table S3. Bond lengths $[\AA]$ and angles $\left[{ }^{\circ}\right]$ for $(-)-9$ in $P 2_{1} \mathbf{2}_{1} \mathbf{2}$.

\begin{tabular}{|c|c|c|c|}
\hline $\mathrm{S}(1)-\mathrm{O}(1)$ & $1.434(2)$ & $\mathrm{C}(13)-\mathrm{H}(13)$ & 0.9500 \\
\hline $\mathrm{S}(1)-\mathrm{O}(2)$ & $1.441(2)$ & $C(14)-C(15)$ & $1.385(5)$ \\
\hline $\mathrm{S}(1)-\mathrm{N}(1)$ & $1.610(2)$ & $C(14)-C(17)$ & $1.513(5)$ \\
\hline$S(1)-C(11)$ & $1.767(3)$ & $C(15)-C(16)$ & $1.383(4)$ \\
\hline $\mathrm{N}(1)-\mathrm{C}(10)$ & $1.480(4)$ & $\mathrm{C}(15)-\mathrm{H}(15)$ & 0.9500 \\
\hline $\mathrm{N}(1)-\mathrm{H}(1)$ & $0.84(2)$ & $\mathrm{C}(16)-\mathrm{H}(16)$ & 0.9500 \\
\hline $\mathrm{C}(1)-\mathrm{C}(2)$ & $1.297(5)$ & $\mathrm{C}(17)-\mathrm{H}(17 \mathrm{~A})$ & 0.9800 \\
\hline $\mathrm{C}(1)-\mathrm{H}(1 \mathrm{~A})$ & 0.9500 & $\mathrm{C}(17)-\mathrm{H}(17 \mathrm{~B})$ & 0.9800 \\
\hline $\mathrm{C}(1)-\mathrm{H}(1 \mathrm{~B})$ & 0.9500 & $\mathrm{C}(17)-\mathrm{H}(17 \mathrm{C})$ & 0.9800 \\
\hline $\mathrm{C}(2)-\mathrm{C}(3)$ & $1.491(5)$ & $\mathrm{S}(21)-\mathrm{O}(21)$ & $1.429(2)$ \\
\hline $\mathrm{C}(2)-\mathrm{H}(2)$ & 0.9500 & $\mathrm{~S}(21)-\mathrm{O}(22)$ & $1.4431(19)$ \\
\hline$C(3)-C(4)$ & $1.540(4)$ & $\mathrm{S}(21)-\mathrm{N}(21)$ & $1.614(2)$ \\
\hline $\mathrm{C}(3)-\mathrm{H}(3 \mathrm{~A})$ & 0.9900 & $\mathrm{~S}(21)-\mathrm{C}(31)$ & $1.759(3)$ \\
\hline $\mathrm{C}(3)-\mathrm{H}(3 \mathrm{~B})$ & 0.9900 & $\mathrm{~N}(21)-\mathrm{C}(30)$ & $1.459(7)$ \\
\hline$C(4)-C(10)$ & $1.532(4)$ & $\mathrm{N}(21)-\mathrm{C}(30 \mathrm{~A})$ & $1.55(3)$ \\
\hline$C(4)-C(5)$ & $1.535(4)$ & $\mathrm{N}(21)-\mathrm{H}(21)$ & $0.85(2)$ \\
\hline $\mathrm{C}(4)-\mathrm{H}(4)$ & 1.0000 & $C(21)-C(22)$ & $1.309(5)$ \\
\hline$C(5)-C(6)$ & $1.533(5)$ & $\mathrm{C}(21)-\mathrm{H}(21 \mathrm{~A})$ & 0.9500 \\
\hline $\mathrm{C}(5)-\mathrm{H}(5 \mathrm{~A})$ & 0.9900 & $\mathrm{C}(21)-\mathrm{H}(21 \mathrm{~B})$ & 0.9500 \\
\hline $\mathrm{C}(5)-\mathrm{H}(5 \mathrm{~B})$ & 0.9900 & $C(22)-C(23)$ & $1.490(4)$ \\
\hline$C(6)-C(7)$ & $1.526(6)$ & $\mathrm{C}(22)-\mathrm{H}(22)$ & 0.9500 \\
\hline $\mathrm{C}(6)-\mathrm{H}(6 \mathrm{~A})$ & 0.9900 & $C(23)-C(24)$ & $1.537(4)$ \\
\hline $\mathrm{C}(6)-\mathrm{H}(6 \mathrm{~B})$ & 0.9900 & $\mathrm{C}(23)-\mathrm{H}(23 \mathrm{~A})$ & 0.9900 \\
\hline$C(7)-C(8)$ & $1.530(5)$ & $\mathrm{C}(23)-\mathrm{H}(23 \mathrm{~B})$ & 0.9900 \\
\hline $\mathrm{C}(7)-\mathrm{H}(7 \mathrm{~A})$ & 0.9900 & $C(24)-C(25 A)$ & $1.46(7)$ \\
\hline $\mathrm{C}(7)-\mathrm{H}(7 \mathrm{~B})$ & 0.9900 & $C(24)-C(30 A)$ & $1.52(3)$ \\
\hline $\mathrm{C}(8)-\mathrm{C}(9)$ & $1.531(5)$ & $C(24)-C(25)$ & $1.547(17)$ \\
\hline $\mathrm{C}(8)-\mathrm{H}(8 \mathrm{~A})$ & 0.9900 & $C(24)-C(30)$ & $1.553(8)$ \\
\hline $\mathrm{C}(8)-\mathrm{H}(8 \mathrm{~B})$ & 0.9900 & $\mathrm{C}(24)-\mathrm{H}(24)$ & 1.0000 \\
\hline$C(9)-C(10)$ & $1.531(4)$ & $\mathrm{C}(24)-\mathrm{H}(24 \mathrm{~A})$ & 1.0000 \\
\hline $\mathrm{C}(9)-\mathrm{H}(9 \mathrm{~A})$ & 0.9900 & $C(25)-C(26)$ & $1.529(8)$ \\
\hline $\mathrm{C}(9)-\mathrm{H}(9 \mathrm{~B})$ & 0.9900 & $\mathrm{C}(25)-\mathrm{H}(25 \mathrm{~A})$ & 0.9900 \\
\hline $\mathrm{C}(10)-\mathrm{H}(10)$ & 1.0000 & $\mathrm{C}(25)-\mathrm{H}(25 \mathrm{~B})$ & 0.9900 \\
\hline $\mathrm{C}(11)-\mathrm{C}(12)$ & $1.389(4)$ & $C(26)-C(27)$ & $1.526(6)$ \\
\hline$C(11)-C(16)$ & $1.390(4)$ & $\mathrm{C}(26)-\mathrm{H}(26 \mathrm{~A})$ & 0.9900 \\
\hline$C(12)-C(13)$ & $1.380(4)$ & $\mathrm{C}(26)-\mathrm{H}(26 \mathrm{~B})$ & 0.9900 \\
\hline $\mathrm{C}(12)-\mathrm{H}(12)$ & 0.9500 & $C(27)-C(28)$ & $1.521(7)$ \\
\hline $\mathrm{C}(13)-\mathrm{C}(14)$ & $1.389(5)$ & $\mathrm{C}(27)-\mathrm{H}(27 \mathrm{~A})$ & 0.9900 \\
\hline
\end{tabular}




\begin{tabular}{|c|c|c|c|}
\hline $\mathrm{C}(27)-\mathrm{H}(27 \mathrm{~B})$ & 0.9900 & $\mathrm{O}(1)-\mathrm{S}(1)-\mathrm{N}(1)$ & $107.73(13)$ \\
\hline $\mathrm{C}(28)-\mathrm{C}(29)$ & $1.537(6)$ & $\mathrm{O}(2)-\mathrm{S}(1)-\mathrm{N}(1)$ & $105.74(12)$ \\
\hline $\mathrm{C}(28)-\mathrm{H}(28 \mathrm{~A})$ & 0.9900 & $\mathrm{O}(1)-\mathrm{S}(1)-\mathrm{C}(11)$ & $107.60(13)$ \\
\hline $\mathrm{C}(28)-\mathrm{H}(28 \mathrm{~B})$ & 0.9900 & $\mathrm{O}(2)-\mathrm{S}(1)-\mathrm{C}(11)$ & $107.27(13)$ \\
\hline C(29)-C(30) & $1.533(6)$ & $\mathrm{N}(1)-\mathrm{S}(1)-\mathrm{C}(11)$ & $108.30(13)$ \\
\hline C(29)-H(29A) & 0.9900 & $C(10)-N(1)-S(1)$ & $121.14(19)$ \\
\hline C(29)-H(29B) & 0.9900 & $\mathrm{C}(10)-\mathrm{N}(1)-\mathrm{H}(1)$ & $118(2)$ \\
\hline $\mathrm{C}(30)-\mathrm{H}(30)$ & 1.0000 & $\mathrm{~S}(1)-\mathrm{N}(1)-\mathrm{H}(1)$ & $113(2)$ \\
\hline$C(25 A)-C(26 A)$ & $1.54(2)$ & $\mathrm{C}(2)-\mathrm{C}(1)-\mathrm{H}(1 \mathrm{~A})$ & 120.0 \\
\hline $\mathrm{C}(25 \mathrm{~A})-\mathrm{H}(25 \mathrm{C})$ & 0.9900 & $\mathrm{C}(2)-\mathrm{C}(1)-\mathrm{H}(1 \mathrm{~B})$ & 120.0 \\
\hline $\mathrm{C}(25 \mathrm{~A})-\mathrm{H}(25 \mathrm{D})$ & 0.9900 & $\mathrm{H}(1 \mathrm{~A})-\mathrm{C}(1)-\mathrm{H}(1 \mathrm{~B})$ & 120.0 \\
\hline$C(26 A)-C(27 A)$ & $1.52(2)$ & $\mathrm{C}(1)-\mathrm{C}(2)-\mathrm{C}(3)$ & $126.3(3)$ \\
\hline $\mathrm{C}(26 \mathrm{~A})-\mathrm{H}(26 \mathrm{C})$ & 0.9900 & $\mathrm{C}(1)-\mathrm{C}(2)-\mathrm{H}(2)$ & 116.8 \\
\hline $\mathrm{C}(26 \mathrm{~A})-\mathrm{H}(26 \mathrm{D})$ & 0.9900 & $\mathrm{C}(3)-\mathrm{C}(2)-\mathrm{H}(2)$ & 116.8 \\
\hline $\mathrm{C}(27 \mathrm{~A})-\mathrm{C}(28 \mathrm{~A})$ & $1.51(2)$ & $C(2)-C(3)-C(4)$ & $113.6(3)$ \\
\hline $\mathrm{C}(27 \mathrm{~A})-\mathrm{H}(27 \mathrm{C})$ & 0.9900 & $\mathrm{C}(2)-\mathrm{C}(3)-\mathrm{H}(3 \mathrm{~A})$ & 108.9 \\
\hline $\mathrm{C}(27 \mathrm{~A})-\mathrm{H}(27 \mathrm{D})$ & 0.9900 & $\mathrm{C}(4)-\mathrm{C}(3)-\mathrm{H}(3 \mathrm{~A})$ & 108.9 \\
\hline$C(28 A)-C(29 A)$ & $1.532(17)$ & $\mathrm{C}(2)-\mathrm{C}(3)-\mathrm{H}(3 \mathrm{~B})$ & 108.9 \\
\hline $\mathrm{C}(28 \mathrm{~A})-\mathrm{H}(28 \mathrm{C})$ & 0.9900 & $\mathrm{C}(4)-\mathrm{C}(3)-\mathrm{H}(3 \mathrm{~B})$ & 108.9 \\
\hline $\mathrm{C}(28 \mathrm{~A})-\mathrm{H}(28 \mathrm{D})$ & 0.9900 & $\mathrm{H}(3 \mathrm{~A})-\mathrm{C}(3)-\mathrm{H}(3 \mathrm{~B})$ & 107.7 \\
\hline$C(29 A)-C(30 A)$ & $1.54(2)$ & $\mathrm{C}(10)-\mathrm{C}(4)-\mathrm{C}(5)$ & $115.5(3)$ \\
\hline $\mathrm{C}(29 \mathrm{~A})-\mathrm{H}(29 \mathrm{C})$ & 0.9900 & $\mathrm{C}(10)-\mathrm{C}(4)-\mathrm{C}(3)$ & $111.3(3)$ \\
\hline $\mathrm{C}(29 \mathrm{~A})-\mathrm{H}(29 \mathrm{D})$ & 0.9900 & $C(5)-C(4)-C(3)$ & $111.8(3)$ \\
\hline $\mathrm{C}(30 \mathrm{~A})-\mathrm{H}(30 \mathrm{~A})$ & 1.0000 & $\mathrm{C}(10)-\mathrm{C}(4)-\mathrm{H}(4)$ & 105.8 \\
\hline$C(31)-C(32)$ & $1.384(4)$ & $\mathrm{C}(5)-\mathrm{C}(4)-\mathrm{H}(4)$ & 105.8 \\
\hline$C(31)-C(36)$ & $1.385(4)$ & $\mathrm{C}(3)-\mathrm{C}(4)-\mathrm{H}(4)$ & 105.8 \\
\hline$C(32)-C(33)$ & $1.379(4)$ & $C(6)-C(5)-C(4)$ & $113.5(3)$ \\
\hline $\mathrm{C}(32)-\mathrm{H}(32)$ & 0.9500 & $\mathrm{C}(6)-\mathrm{C}(5)-\mathrm{H}(5 \mathrm{~A})$ & 108.9 \\
\hline$C(33)-C(34)$ & $1.386(4)$ & $\mathrm{C}(4)-\mathrm{C}(5)-\mathrm{H}(5 \mathrm{~A})$ & 108.9 \\
\hline $\mathrm{C}(33)-\mathrm{H}(33)$ & 0.9500 & $\mathrm{C}(6)-\mathrm{C}(5)-\mathrm{H}(5 \mathrm{~B})$ & 108.9 \\
\hline$C(34)-C(35)$ & $1.393(4)$ & $\mathrm{C}(4)-\mathrm{C}(5)-\mathrm{H}(5 \mathrm{~B})$ & 108.9 \\
\hline $\mathrm{C}(34)-\mathrm{C}(37)$ & $1.504(4)$ & $\mathrm{H}(5 \mathrm{~A})-\mathrm{C}(5)-\mathrm{H}(5 \mathrm{~B})$ & 107.7 \\
\hline$C(35)-C(36)$ & $1.382(4)$ & $C(7)-C(6)-C(5)$ & $116.4(3)$ \\
\hline $\mathrm{C}(35)-\mathrm{H}(35)$ & 0.9500 & $\mathrm{C}(7)-\mathrm{C}(6)-\mathrm{H}(6 \mathrm{~A})$ & 108.2 \\
\hline $\mathrm{C}(36)-\mathrm{H}(36)$ & 0.9500 & $\mathrm{C}(5)-\mathrm{C}(6)-\mathrm{H}(6 \mathrm{~A})$ & 108.2 \\
\hline $\mathrm{C}(37)-\mathrm{H}(37 \mathrm{~A})$ & 0.9800 & $\mathrm{C}(7)-\mathrm{C}(6)-\mathrm{H}(6 \mathrm{~B})$ & 108.2 \\
\hline C(37)-H(37B) & 0.9800 & $\mathrm{C}(5)-\mathrm{C}(6)-\mathrm{H}(6 \mathrm{~B})$ & 108.2 \\
\hline \multirow[t]{2}{*}{$\mathrm{C}(37)-\mathrm{H}(37 \mathrm{C})$} & 0.9800 & $\mathrm{H}(6 \mathrm{~A})-\mathrm{C}(6)-\mathrm{H}(6 \mathrm{~B})$ & 107.3 \\
\hline & & $\mathrm{C}(6)-\mathrm{C}(7)-\mathrm{C}(8)$ & $116.0(3)$ \\
\hline $\mathrm{O}(1)-\mathrm{S}(1)-\mathrm{O}(2)$ & $119.78(13)$ & $\mathrm{C}(6)-\mathrm{C}(7)-\mathrm{H}(7 \mathrm{~A})$ & 108.3 \\
\hline
\end{tabular}




\begin{tabular}{|c|c|c|c|}
\hline $\mathrm{C}(8)-\mathrm{C}(7)-\mathrm{H}(7 \mathrm{~A})$ & 108.3 & $\mathrm{C}(14)-\mathrm{C}(17)-\mathrm{H}(17 \mathrm{~A})$ & 109.5 \\
\hline $\mathrm{C}(6)-\mathrm{C}(7)-\mathrm{H}(7 \mathrm{~B})$ & 108.3 & $\mathrm{C}(14)-\mathrm{C}(17)-\mathrm{H}(17 \mathrm{~B})$ & 109.5 \\
\hline $\mathrm{C}(8)-\mathrm{C}(7)-\mathrm{H}(7 \mathrm{~B})$ & 108.3 & $\mathrm{H}(17 \mathrm{~A})-\mathrm{C}(17)-\mathrm{H}(17 \mathrm{~B})$ & 109.5 \\
\hline $\mathrm{H}(7 \mathrm{~A})-\mathrm{C}(7)-\mathrm{H}(7 \mathrm{~B})$ & 107.4 & $\mathrm{C}(14)-\mathrm{C}(17)-\mathrm{H}(17 \mathrm{C})$ & 109.5 \\
\hline $\mathrm{C}(7)-\mathrm{C}(8)-\mathrm{C}(9)$ & $115.6(3)$ & $\mathrm{H}(17 \mathrm{~A})-\mathrm{C}(17)-\mathrm{H}(17 \mathrm{C})$ & 109.5 \\
\hline $\mathrm{C}(7)-\mathrm{C}(8)-\mathrm{H}(8 \mathrm{~A})$ & 108.4 & $\mathrm{H}(17 \mathrm{~B})-\mathrm{C}(17)-\mathrm{H}(17 \mathrm{C})$ & 109.5 \\
\hline $\mathrm{C}(9)-\mathrm{C}(8)-\mathrm{H}(8 \mathrm{~A})$ & 108.4 & $\mathrm{O}(21)-\mathrm{S}(21)-\mathrm{O}(22)$ & $119.34(13)$ \\
\hline $\mathrm{C}(7)-\mathrm{C}(8)-\mathrm{H}(8 \mathrm{~B})$ & 108.4 & $\mathrm{O}(21)-\mathrm{S}(21)-\mathrm{N}(21)$ & $108.55(12)$ \\
\hline $\mathrm{C}(9)-\mathrm{C}(8)-\mathrm{H}(8 \mathrm{~B})$ & 108.4 & $\mathrm{O}(22)-\mathrm{S}(21)-\mathrm{N}(21)$ & $105.61(12)$ \\
\hline $\mathrm{H}(8 \mathrm{~A})-\mathrm{C}(8)-\mathrm{H}(8 \mathrm{~B})$ & 107.5 & $\mathrm{O}(21)-\mathrm{S}(21)-\mathrm{C}(31)$ & $107.82(13)$ \\
\hline $\mathrm{C}(10)-\mathrm{C}(9)-\mathrm{C}(8)$ & $114.8(3)$ & $\mathrm{O}(22)-\mathrm{S}(21)-\mathrm{C}(31)$ & $107.57(13)$ \\
\hline $\mathrm{C}(10)-\mathrm{C}(9)-\mathrm{H}(9 \mathrm{~A})$ & 108.6 & $\mathrm{~N}(21)-\mathrm{S}(21)-\mathrm{C}(31)$ & $107.42(13)$ \\
\hline $\mathrm{C}(8)-\mathrm{C}(9)-\mathrm{H}(9 \mathrm{~A})$ & 108.6 & $\mathrm{C}(30)-\mathrm{N}(21)-\mathrm{S}(21)$ & $123.6(3)$ \\
\hline $\mathrm{C}(10)-\mathrm{C}(9)-\mathrm{H}(9 \mathrm{~B})$ & 108.6 & $\mathrm{C}(30 \mathrm{~A})-\mathrm{N}(21)-\mathrm{S}(21)$ & $117.6(10)$ \\
\hline $\mathrm{C}(8)-\mathrm{C}(9)-\mathrm{H}(9 \mathrm{~B})$ & 108.6 & $\mathrm{C}(30)-\mathrm{N}(21)-\mathrm{H}(21)$ & $115(2)$ \\
\hline $\mathrm{H}(9 \mathrm{~A})-\mathrm{C}(9)-\mathrm{H}(9 \mathrm{~B})$ & 107.5 & $\mathrm{C}(30 \mathrm{~A})-\mathrm{N}(21)-\mathrm{H}(21)$ & $123(2)$ \\
\hline $\mathrm{N}(1)-\mathrm{C}(10)-\mathrm{C}(9)$ & $109.2(2)$ & $\mathrm{S}(21)-\mathrm{N}(21)-\mathrm{H}(21)$ & $111(2)$ \\
\hline $\mathrm{N}(1)-\mathrm{C}(10)-\mathrm{C}(4)$ & $110.7(2)$ & $\mathrm{C}(22)-\mathrm{C}(21)-\mathrm{H}(21 \mathrm{~A})$ & 120.0 \\
\hline $\mathrm{C}(9)-\mathrm{C}(10)-\mathrm{C}(4)$ & $116.0(3)$ & $\mathrm{C}(22)-\mathrm{C}(21)-\mathrm{H}(21 \mathrm{~B})$ & 120.0 \\
\hline $\mathrm{N}(1)-\mathrm{C}(10)-\mathrm{H}(10)$ & 106.8 & $\mathrm{H}(21 \mathrm{~A})-\mathrm{C}(21)-\mathrm{H}(21 \mathrm{~B})$ & 120.0 \\
\hline $\mathrm{C}(9)-\mathrm{C}(10)-\mathrm{H}(10)$ & 106.8 & $\mathrm{C}(21)-\mathrm{C}(22)-\mathrm{C}(23)$ & $125.1(3)$ \\
\hline $\mathrm{C}(4)-\mathrm{C}(10)-\mathrm{H}(10)$ & 106.8 & $\mathrm{C}(21)-\mathrm{C}(22)-\mathrm{H}(22)$ & 117.5 \\
\hline $\mathrm{C}(12)-\mathrm{C}(11)-\mathrm{C}(16)$ & $120.3(3)$ & $\mathrm{C}(23)-\mathrm{C}(22)-\mathrm{H}(22)$ & 117.5 \\
\hline $\mathrm{C}(12)-\mathrm{C}(11)-\mathrm{S}(1)$ & $119.6(2)$ & $\mathrm{C}(22)-\mathrm{C}(23)-\mathrm{C}(24)$ & $113.6(2)$ \\
\hline $\mathrm{C}(16)-\mathrm{C}(11)-\mathrm{S}(1)$ & $120.1(2)$ & $\mathrm{C}(22)-\mathrm{C}(23)-\mathrm{H}(23 \mathrm{~A})$ & 108.8 \\
\hline $\mathrm{C}(13)-\mathrm{C}(12)-\mathrm{C}(11)$ & $119.4(3)$ & $\mathrm{C}(24)-\mathrm{C}(23)-\mathrm{H}(23 \mathrm{~A})$ & 108.8 \\
\hline $\mathrm{C}(13)-\mathrm{C}(12)-\mathrm{H}(12)$ & 120.3 & $\mathrm{C}(22)-\mathrm{C}(23)-\mathrm{H}(23 \mathrm{~B})$ & 108.8 \\
\hline $\mathrm{C}(11)-\mathrm{C}(12)-\mathrm{H}(12)$ & 120.3 & $\mathrm{C}(24)-\mathrm{C}(23)-\mathrm{H}(23 \mathrm{~B})$ & 108.8 \\
\hline $\mathrm{C}(12)-\mathrm{C}(13)-\mathrm{C}(14)$ & $121.2(3)$ & $\mathrm{H}(23 \mathrm{~A})-\mathrm{C}(23)-\mathrm{H}(23 \mathrm{~B})$ & 107.7 \\
\hline $\mathrm{C}(12)-\mathrm{C}(13)-\mathrm{H}(13)$ & 119.4 & $C(25 \mathrm{~A})-\mathrm{C}(24)-\mathrm{C}(30 \mathrm{~A})$ & $120(3)$ \\
\hline $\mathrm{C}(14)-\mathrm{C}(13)-\mathrm{H}(13)$ & 119.4 & $\mathrm{C}(25 \mathrm{~A})-\mathrm{C}(24)-\mathrm{C}(23)$ & $116.3(17)$ \\
\hline$C(15)-C(14)-C(13)$ & $118.6(3)$ & $C(30 A)-C(24)-C(23)$ & $105.4(9)$ \\
\hline $\mathrm{C}(15)-\mathrm{C}(14)-\mathrm{C}(17)$ & $120.4(3)$ & $C(23)-C(24)-C(25)$ & $111.9(4)$ \\
\hline $\mathrm{C}(13)-\mathrm{C}(14)-\mathrm{C}(17)$ & $121.0(3)$ & $\mathrm{C}(23)-\mathrm{C}(24)-\mathrm{C}(30)$ & $110.5(3)$ \\
\hline $\mathrm{C}(16)-\mathrm{C}(15)-\mathrm{C}(14)$ & $121.3(3)$ & $\mathrm{C}(25)-\mathrm{C}(24)-\mathrm{C}(30)$ & $112.0(8)$ \\
\hline $\mathrm{C}(16)-\mathrm{C}(15)-\mathrm{H}(15)$ & 119.4 & $\mathrm{C}(23)-\mathrm{C}(24)-\mathrm{H}(24)$ & 107.4 \\
\hline $\mathrm{C}(14)-\mathrm{C}(15)-\mathrm{H}(15)$ & 119.4 & $\mathrm{C}(25)-\mathrm{C}(24)-\mathrm{H}(24)$ & 107.4 \\
\hline $\mathrm{C}(15)-\mathrm{C}(16)-\mathrm{C}(11)$ & 119.3(3) & $\mathrm{C}(30)-\mathrm{C}(24)-\mathrm{H}(24)$ & 107.4 \\
\hline $\mathrm{C}(15)-\mathrm{C}(16)-\mathrm{H}(16)$ & 120.4 & $\mathrm{C}(25 \mathrm{~A})-\mathrm{C}(24)-\mathrm{H}(24 \mathrm{~A})$ & 104.6 \\
\hline $\mathrm{C}(11)-\mathrm{C}(16)-\mathrm{H}(16)$ & 120.4 & $\mathrm{C}(30 \mathrm{~A})-\mathrm{C}(24)-\mathrm{H}(24 \mathrm{~A})$ & 104.6 \\
\hline
\end{tabular}




\begin{tabular}{|c|c|c|c|}
\hline $\mathrm{C}(23)-\mathrm{C}(24)-\mathrm{H}(24 \mathrm{~A})$ & 104.6 & $\mathrm{C}(24)-\mathrm{C}(25 \mathrm{~A})-\mathrm{H}(25 \mathrm{D})$ & 108.7 \\
\hline$C(26)-C(25)-C(24)$ & $113.6(12)$ & $C(26 A)-C(25 A)-H(25 D)$ & 108.7 \\
\hline $\mathrm{C}(26)-\mathrm{C}(25)-\mathrm{H}(25 \mathrm{~A})$ & 108.9 & $\mathrm{H}(25 \mathrm{C})-\mathrm{C}(25 \mathrm{~A})-\mathrm{H}(25 \mathrm{D})$ & 107.6 \\
\hline $\mathrm{C}(24)-\mathrm{C}(25)-\mathrm{H}(25 \mathrm{~A})$ & 108.9 & $C(27 A)-C(26 A)-C(25 A)$ & $114(3)$ \\
\hline $\mathrm{C}(26)-\mathrm{C}(25)-\mathrm{H}(25 \mathrm{~B})$ & 108.9 & $\mathrm{C}(27 \mathrm{~A})-\mathrm{C}(26 \mathrm{~A})-\mathrm{H}(26 \mathrm{C})$ & 108.7 \\
\hline $\mathrm{C}(24)-\mathrm{C}(25)-\mathrm{H}(25 \mathrm{~B})$ & 108.9 & $\mathrm{C}(25 \mathrm{~A})-\mathrm{C}(26 \mathrm{~A})-\mathrm{H}(26 \mathrm{C})$ & 108.7 \\
\hline $\mathrm{H}(25 \mathrm{~A})-\mathrm{C}(25)-\mathrm{H}(25 \mathrm{~B})$ & 107.7 & $\mathrm{C}(27 \mathrm{~A})-\mathrm{C}(26 \mathrm{~A})-\mathrm{H}(26 \mathrm{D})$ & 108.7 \\
\hline$C(27)-C(26)-C(25)$ & $115.7(7)$ & $C(25 A)-C(26 A)-H(26 D)$ & 108.7 \\
\hline $\mathrm{C}(27)-\mathrm{C}(26)-\mathrm{H}(26 \mathrm{~A})$ & 108.4 & $\mathrm{H}(26 \mathrm{C})-\mathrm{C}(26 \mathrm{~A})-\mathrm{H}(26 \mathrm{D})$ & 107.6 \\
\hline $\mathrm{C}(25)-\mathrm{C}(26)-\mathrm{H}(26 \mathrm{~A})$ & 108.4 & $\mathrm{C}(28 \mathrm{~A})-\mathrm{C}(27 \mathrm{~A})-\mathrm{C}(26 \mathrm{~A})$ & $119(2)$ \\
\hline $\mathrm{C}(27)-\mathrm{C}(26)-\mathrm{H}(26 \mathrm{~B})$ & 108.4 & $\mathrm{C}(28 \mathrm{~A})-\mathrm{C}(27 \mathrm{~A})-\mathrm{H}(27 \mathrm{C})$ & 107.6 \\
\hline $\mathrm{C}(25)-\mathrm{C}(26)-\mathrm{H}(26 \mathrm{~B})$ & 108.4 & $\mathrm{C}(26 \mathrm{~A})-\mathrm{C}(27 \mathrm{~A})-\mathrm{H}(27 \mathrm{C})$ & 107.6 \\
\hline $\mathrm{H}(26 \mathrm{~A})-\mathrm{C}(26)-\mathrm{H}(26 \mathrm{~B})$ & 107.4 & $\mathrm{C}(28 \mathrm{~A})-\mathrm{C}(27 \mathrm{~A})-\mathrm{H}(27 \mathrm{D})$ & 107.6 \\
\hline$C(28)-C(27)-C(26)$ & $117.3(5)$ & $\mathrm{C}(26 \mathrm{~A})-\mathrm{C}(27 \mathrm{~A})-\mathrm{H}(27 \mathrm{D})$ & 107.6 \\
\hline $\mathrm{C}(28)-\mathrm{C}(27)-\mathrm{H}(27 \mathrm{~A})$ & 108.0 & $\mathrm{H}(27 \mathrm{C})-\mathrm{C}(27 \mathrm{~A})-\mathrm{H}(27 \mathrm{D})$ & 107.0 \\
\hline $\mathrm{C}(26)-\mathrm{C}(27)-\mathrm{H}(27 \mathrm{~A})$ & 108.0 & $\mathrm{C}(27 \mathrm{~A})-\mathrm{C}(28 \mathrm{~A})-\mathrm{C}(29 \mathrm{~A})$ & $116.2(19)$ \\
\hline $\mathrm{C}(28)-\mathrm{C}(27)-\mathrm{H}(27 \mathrm{~B})$ & 108.0 & $\mathrm{C}(27 \mathrm{~A})-\mathrm{C}(28 \mathrm{~A})-\mathrm{H}(28 \mathrm{C})$ & 108.2 \\
\hline $\mathrm{C}(26)-\mathrm{C}(27)-\mathrm{H}(27 \mathrm{~B})$ & 108.0 & $\mathrm{C}(29 \mathrm{~A})-\mathrm{C}(28 \mathrm{~A})-\mathrm{H}(28 \mathrm{C})$ & 108.2 \\
\hline $\mathrm{H}(27 \mathrm{~A})-\mathrm{C}(27)-\mathrm{H}(27 \mathrm{~B})$ & 107.2 & $\mathrm{C}(27 \mathrm{~A})-\mathrm{C}(28 \mathrm{~A})-\mathrm{H}(28 \mathrm{D})$ & 108.2 \\
\hline $\mathrm{C}(27)-\mathrm{C}(28)-\mathrm{C}(29)$ & $113.6(4)$ & $\mathrm{C}(29 \mathrm{~A})-\mathrm{C}(28 \mathrm{~A})-\mathrm{H}(28 \mathrm{D})$ & 108.2 \\
\hline $\mathrm{C}(27)-\mathrm{C}(28)-\mathrm{H}(28 \mathrm{~A})$ & 108.8 & $\mathrm{H}(28 \mathrm{C})-\mathrm{C}(28 \mathrm{~A})-\mathrm{H}(28 \mathrm{D})$ & 107.4 \\
\hline $\mathrm{C}(29)-\mathrm{C}(28)-\mathrm{H}(28 \mathrm{~A})$ & 108.8 & $C(28 \mathrm{~A})-\mathrm{C}(29 \mathrm{~A})-\mathrm{C}(30 \mathrm{~A})$ & $115.9(17)$ \\
\hline $\mathrm{C}(27)-\mathrm{C}(28)-\mathrm{H}(28 \mathrm{~B})$ & 108.8 & $\mathrm{C}(28 \mathrm{~A})-\mathrm{C}(29 \mathrm{~A})-\mathrm{H}(29 \mathrm{C})$ & 108.3 \\
\hline C(29)-C(28)-H(28B) & 108.8 & $\mathrm{C}(30 \mathrm{~A})-\mathrm{C}(29 \mathrm{~A})-\mathrm{H}(29 \mathrm{C})$ & 108.3 \\
\hline $\mathrm{H}(28 \mathrm{~A})-\mathrm{C}(28)-\mathrm{H}(28 \mathrm{~B})$ & 107.7 & $\mathrm{C}(28 \mathrm{~A})-\mathrm{C}(29 \mathrm{~A})-\mathrm{H}(29 \mathrm{D})$ & 108.3 \\
\hline C(30)-C(29)-C(28) & $117.4(5)$ & $\mathrm{C}(30 \mathrm{~A})-\mathrm{C}(29 \mathrm{~A})-\mathrm{H}(29 \mathrm{D})$ & 108.3 \\
\hline $\mathrm{C}(30)-\mathrm{C}(29)-\mathrm{H}(29 \mathrm{~A})$ & 108.0 & $\mathrm{H}(29 \mathrm{C})-\mathrm{C}(29 \mathrm{~A})-\mathrm{H}(29 \mathrm{D})$ & 107.4 \\
\hline $\mathrm{C}(28)-\mathrm{C}(29)-\mathrm{H}(29 \mathrm{~A})$ & 108.0 & $C(24)-C(30 A)-C(29 A)$ & $115(2)$ \\
\hline C(30)-C(29)-H(29B) & 108.0 & $\mathrm{C}(24)-\mathrm{C}(30 \mathrm{~A})-\mathrm{N}(21)$ & $106.9(16)$ \\
\hline C(28)-C(29)-H(29B) & 108.0 & $\mathrm{C}(29 \mathrm{~A})-\mathrm{C}(30 \mathrm{~A})-\mathrm{N}(21)$ & $110.8(18)$ \\
\hline $\mathrm{H}(29 \mathrm{~A})-\mathrm{C}(29)-\mathrm{H}(29 \mathrm{~B})$ & 107.2 & $\mathrm{C}(24)-\mathrm{C}(30 \mathrm{~A})-\mathrm{H}(30 \mathrm{~A})$ & 108.1 \\
\hline $\mathrm{N}(21)-\mathrm{C}(30)-\mathrm{C}(29)$ & $110.2(4)$ & $\mathrm{C}(29 \mathrm{~A})-\mathrm{C}(30 \mathrm{~A})-\mathrm{H}(30 \mathrm{~A})$ & 108.1 \\
\hline $\mathrm{N}(21)-\mathrm{C}(30)-\mathrm{C}(24)$ & $109.4(4)$ & $\mathrm{N}(21)-\mathrm{C}(30 \mathrm{~A})-\mathrm{H}(30 \mathrm{~A})$ & 108.1 \\
\hline C(29)-C(30)-C(24) & $115.2(5)$ & $\mathrm{C}(32)-\mathrm{C}(31)-\mathrm{C}(36)$ & $120.0(3)$ \\
\hline $\mathrm{N}(21)-\mathrm{C}(30)-\mathrm{H}(30)$ & 107.2 & $\mathrm{C}(32)-\mathrm{C}(31)-\mathrm{S}(21)$ & $120.4(2)$ \\
\hline $\mathrm{C}(29)-\mathrm{C}(30)-\mathrm{H}(30)$ & 107.2 & $\mathrm{C}(36)-\mathrm{C}(31)-\mathrm{S}(21)$ & $119.6(2)$ \\
\hline $\mathrm{C}(24)-\mathrm{C}(30)-\mathrm{H}(30)$ & 107.2 & $\mathrm{C}(33)-\mathrm{C}(32)-\mathrm{C}(31)$ & $119.7(3)$ \\
\hline$C(24)-C(25 A)-C(26 A)$ & $114(5)$ & $\mathrm{C}(33)-\mathrm{C}(32)-\mathrm{H}(32)$ & 120.1 \\
\hline $\mathrm{C}(24)-\mathrm{C}(25 \mathrm{~A})-\mathrm{H}(25 \mathrm{C})$ & 108.7 & $\mathrm{C}(31)-\mathrm{C}(32)-\mathrm{H}(32)$ & 120.1 \\
\hline $\mathrm{C}(26 \mathrm{~A})-\mathrm{C}(25 \mathrm{~A})-\mathrm{H}(25 \mathrm{C})$ & 108.7 & $\mathrm{C}(32)-\mathrm{C}(33)-\mathrm{C}(34)$ & $121.4(3)$ \\
\hline
\end{tabular}




$\begin{array}{llll}\mathrm{C}(32)-\mathrm{C}(33)-\mathrm{H}(33) & 119.3 & \mathrm{C}(35)-\mathrm{C}(36)-\mathrm{H}(36) & 120.2 \\ \mathrm{C}(34)-\mathrm{C}(33)-\mathrm{H}(33) & 119.3 & \mathrm{C}(31)-\mathrm{C}(36)-\mathrm{H}(36) & 120.2 \\ \mathrm{C}(33)-\mathrm{C}(34)-\mathrm{C}(35) & 118.1(3) & \mathrm{C}(34)-\mathrm{C}(37)-\mathrm{H}(37 \mathrm{~A}) & 109.5 \\ \mathrm{C}(33)-\mathrm{C}(34)-\mathrm{C}(37) & 121.0(3) & \mathrm{C}(34)-\mathrm{C}(37)-\mathrm{H}(37 \mathrm{~B}) & 109.5 \\ \mathrm{C}(35)-\mathrm{C}(34)-\mathrm{C}(37) & 120.9(3) & \mathrm{H}(37 \mathrm{~A})-\mathrm{C}(37)-\mathrm{H}(37 \mathrm{~B}) & 109.5 \\ \mathrm{C}(36)-\mathrm{C}(35)-\mathrm{C}(34) & 121.1(3) & \mathrm{C}(34)-\mathrm{C}(37)-\mathrm{H}(37 \mathrm{C}) & 109.5 \\ \mathrm{C}(36)-\mathrm{C}(35)-\mathrm{H}(35) & 119.4 & \mathrm{H}(37 \mathrm{~A})-\mathrm{C}(37)-\mathrm{H}(37 \mathrm{C}) & 109.5 \\ \mathrm{C}(34)-\mathrm{C}(35)-\mathrm{H}(35) & 119.4 & \mathrm{H}(37 \mathrm{~B})-\mathrm{C}(37)-\mathrm{H}(37 \mathrm{C}) & 109.5 \\ \mathrm{C}(35)-\mathrm{C}(36)-\mathrm{C}(31) & 119.7(3) & & \end{array}$


Table S4. Anisotropic displacement parameters $\left(\AA^{2} \times 10^{3}\right)$ for (-)-9 in $\mathbf{P 2}_{\mathbf{1}} \mathbf{2}_{1} \mathbf{2}$. The anisotropic displacement factor exponent takes the form: $-2 p^{2}\left[h^{2} a^{* 2} U^{11}+\ldots+2 h k a^{*} b^{*} U^{12}\right]$

\begin{tabular}{|c|c|c|c|c|c|c|}
\hline & $\mathrm{U}^{11}$ & $\mathrm{U}^{22}$ & $\mathrm{U}^{33}$ & $\mathrm{U}^{23}$ & $\mathrm{U}^{13}$ & $\mathrm{U}^{12}$ \\
\hline $\mathrm{S}(1)$ & $20(1)$ & $19(1)$ & $18(1)$ & $2(1)$ & $2(1)$ & $3(1)$ \\
\hline $\mathrm{O}(1)$ & $25(1)$ & $25(1)$ & $21(1)$ & $0(1)$ & $4(1)$ & $6(1)$ \\
\hline $\mathrm{O}(2)$ & $24(1)$ & $21(1)$ & $24(1)$ & $5(1)$ & $5(1)$ & $2(1)$ \\
\hline $\mathrm{N}(1)$ & $21(1)$ & $17(1)$ & $23(1)$ & $1(1)$ & $0(1)$ & $4(1)$ \\
\hline $\mathrm{C}(1)$ & $40(2)$ & $38(2)$ & $46(2)$ & $-5(2)$ & $11(2)$ & $-5(2)$ \\
\hline$C(2)$ & $35(2)$ & $36(2)$ & $33(2)$ & $-1(2)$ & $4(2)$ & $-5(2)$ \\
\hline$C(3)$ & $26(2)$ & $30(2)$ & $29(2)$ & $-2(1)$ & $2(1)$ & $-3(1)$ \\
\hline$C(4)$ & $28(2)$ & $24(2)$ & $27(2)$ & $1(1)$ & $-2(1)$ & $-1(1)$ \\
\hline$C(5)$ & $27(2)$ & $28(2)$ & $39(2)$ & $-3(1)$ & $-3(2)$ & $-2(1)$ \\
\hline$C(6)$ & $41(2)$ & $39(2)$ & $45(2)$ & $-2(2)$ & $-13(2)$ & $-7(2)$ \\
\hline$C(7)$ & $61(3)$ & $48(2)$ & $39(2)$ & $-2(2)$ & $-19(2)$ & $-11(2)$ \\
\hline$C(8)$ & $56(2)$ & $33(2)$ & $26(2)$ & $-4(2)$ & $-5(2)$ & $-7(2)$ \\
\hline $\mathrm{C}(9)$ & $44(2)$ & $25(2)$ & $37(2)$ & $-7(2)$ & $6(2)$ & $-1(2)$ \\
\hline$C(10)$ & $24(1)$ & $19(1)$ & $26(2)$ & $3(1)$ & $-1(1)$ & $1(1)$ \\
\hline $\mathrm{C}(11)$ & $17(1)$ & $24(1)$ & $14(1)$ & $1(1)$ & $1(1)$ & $-1(1)$ \\
\hline$C(12)$ & $25(2)$ & $24(2)$ & $23(2)$ & $1(1)$ & $4(1)$ & $2(1)$ \\
\hline $\mathrm{C}(13)$ & $32(2)$ & $30(2)$ & $26(2)$ & $-8(1)$ & $4(2)$ & $4(1)$ \\
\hline$C(14)$ & $28(2)$ & $44(2)$ & $21(2)$ & $-1(1)$ & $2(1)$ & $-2(1)$ \\
\hline$C(15)$ & $21(2)$ & $35(2)$ & $26(2)$ & $6(1)$ & $0(1)$ & $5(1)$ \\
\hline$C(16)$ & $20(1)$ & $25(2)$ & $21(2)$ & $1(1)$ & $2(1)$ & $4(1)$ \\
\hline$C(17)$ & $57(3)$ & $70(3)$ & $25(2)$ & $-8(2)$ & $-2(2)$ & $11(2)$ \\
\hline $\mathrm{S}(21)$ & $20(1)$ & $17(1)$ & $15(1)$ & $-1(1)$ & $-1(1)$ & $0(1)$ \\
\hline $\mathrm{O}(21)$ & $24(1)$ & $22(1)$ & $20(1)$ & $-4(1)$ & $-1(1)$ & $5(1)$ \\
\hline $\mathrm{O}(22)$ & $24(1)$ & $18(1)$ & 19(1) & $3(1)$ & $0(1)$ & $0(1)$ \\
\hline $\mathrm{N}(21)$ & 19(1) & $17(1)$ & 19(1) & $-1(1)$ & $-4(1)$ & $0(1)$ \\
\hline $\mathrm{C}(21)$ & $38(2)$ & $46(2)$ & $24(2)$ & $-6(2)$ & $-3(2)$ & $0(2)$ \\
\hline $\mathrm{C}(22)$ & $27(2)$ & $23(2)$ & $25(2)$ & $-4(1)$ & $7(1)$ & $-4(1)$ \\
\hline $\mathrm{C}(23)$ & $24(2)$ & $23(2)$ & $21(2)$ & $-1(1)$ & $0(1)$ & $-1(1)$ \\
\hline$C(24)$ & $23(1)$ & $17(1)$ & $17(1)$ & $-1(1)$ & $-3(1)$ & $-1(1)$ \\
\hline$C(25)$ & $25(2)$ & $26(5)$ & $16(3)$ & $-2(2)$ & $-4(2)$ & $0(2)$ \\
\hline$C(26)$ & $25(3)$ & $38(3)$ & $18(3)$ & $-4(2)$ & $-3(2)$ & $3(2)$ \\
\hline $\mathrm{C}(27)$ & $27(2)$ & $32(3)$ & $23(3)$ & $0(2)$ & $-2(2)$ & $-8(2)$ \\
\hline$C(28)$ & $36(2)$ & $33(2)$ & $20(2)$ & $8(2)$ & $1(2)$ & $-8(2)$ \\
\hline$C(29)$ & $31(3)$ & $28(3)$ & $15(2)$ & $1(2)$ & $-2(2)$ & $-7(2)$ \\
\hline $\mathrm{C}(30)$ & $23(1)$ & $17(1)$ & $17(1)$ & $-1(1)$ & $-3(1)$ & $-1(1)$ \\
\hline
\end{tabular}




\begin{tabular}{lcccccc}
$\mathrm{C}(25 \mathrm{~A})$ & $25(2)$ & $26(5)$ & $16(3)$ & $-2(2)$ & $-4(2)$ & $0(2)$ \\
$\mathrm{C}(26 \mathrm{~A})$ & $12(10)$ & $43(12)$ & $51(14)$ & $8(11)$ & $-2(10)$ & $-11(9)$ \\
$\mathrm{C}(27 \mathrm{~A})$ & $34(10)$ & $74(17)$ & $38(12)$ & $19(11)$ & $11(9)$ & $-5(10)$ \\
$\mathrm{C}(28 \mathrm{~A})$ & $40(8)$ & $59(11)$ & $18(8)$ & $3(7)$ & $7(6)$ & $10(7)$ \\
$\mathrm{C}(29 \mathrm{~A})$ & $28(9)$ & $29(12)$ & $30(8)$ & $1(8)$ & $2(8)$ & $1(7)$ \\
$\mathrm{C}(30 \mathrm{~A})$ & $23(1)$ & $17(1)$ & $17(1)$ & $-1(1)$ & $-3(1)$ & $-1(1)$ \\
$\mathrm{C}(31)$ & $19(1)$ & $20(1)$ & $12(1)$ & $1(1)$ & $-3(1)$ & $-3(1)$ \\
$\mathrm{C}(32)$ & $29(2)$ & $23(2)$ & $22(2)$ & $-2(1)$ & $-2(1)$ & $7(1)$ \\
$\mathrm{C}(33)$ & $33(2)$ & $31(2)$ & $24(2)$ & $6(1)$ & $-6(1)$ & $10(2)$ \\
$\mathrm{C}(34)$ & $18(1)$ & $31(2)$ & $17(1)$ & $2(1)$ & $0(1)$ & $-5(1)$ \\
$\mathrm{C}(35)$ & $28(2)$ & $23(2)$ & $20(2)$ & $-3(1)$ & $2(1)$ & $2(1)$ \\
$\mathrm{C}(36)$ & $26(2)$ & $18(1)$ & $20(2)$ & $1(1)$ & $-3(1)$ & $3(1)$ \\
$\mathrm{C}(37)$ & $26(2)$ & $39(2)$ & $18(2)$ & $1(1)$ & $-2(1)$ & $-5(1)$ \\
& & & & & & \\
\hline
\end{tabular}


Table S5. Hydrogen coordinates $\left(x 10^{4}\right)$ and isotropic displacement parameters $\left(\AA^{2} \times 10^{3}\right)$ for $(-)-\mathbf{9}$ in $\boldsymbol{P 2}_{1} \mathbf{2}_{1} \mathbf{2}$.

\begin{tabular}{|c|c|c|c|c|}
\hline & $\mathrm{x}$ & $\mathrm{y}$ & z & $\mathrm{U}(\mathrm{eq})$ \\
\hline $\mathrm{H}(1)$ & $3958(18)$ & $7730(13)$ & $6730(30)$ & 25 \\
\hline $\mathrm{H}(1 \mathrm{~A})$ & 5724 & 7775 & 2922 & 50 \\
\hline $\mathrm{H}(1 \mathrm{~B})$ & 5950 & 8366 & 1920 & 50 \\
\hline $\mathrm{H}(2)$ & 4969 & 8938 & 2914 & 42 \\
\hline $\mathrm{H}(3 \mathrm{~A})$ & 4597 & 7872 & 4562 & 34 \\
\hline $\mathrm{H}(3 \mathrm{~B})$ & 3884 & 8354 & 4077 & 34 \\
\hline $\mathrm{H}(4)$ & 4657 & 9141 & 5395 & 32 \\
\hline $\mathrm{H}(5 \mathrm{~A})$ & 5384 & 8141 & 6892 & 37 \\
\hline $\mathrm{H}(5 \mathrm{~B})$ & 5892 & 8471 & 5682 & 37 \\
\hline $\mathrm{H}(6 \mathrm{~A})$ & 6386 & 9024 & 7414 & 50 \\
\hline $\mathrm{H}(6 \mathrm{~B})$ & 5606 & 9455 & 6982 & 50 \\
\hline $\mathrm{H}(7 \mathrm{~A})$ & 5443 & 9434 & 9217 & 59 \\
\hline $\mathrm{H}(7 \mathrm{~B})$ & 5788 & 8737 & 9355 & 59 \\
\hline $\mathrm{H}(8 \mathrm{~A})$ & 4294 & 8880 & 9883 & 46 \\
\hline $\mathrm{H}(8 \mathrm{~B})$ & 4506 & 8313 & 8890 & 46 \\
\hline $\mathrm{H}(9 \mathrm{~A})$ & 3292 & 9031 & 8343 & 42 \\
\hline $\mathrm{H}(9 \mathrm{~B})$ & 4016 & 9466 & 7714 & 42 \\
\hline $\mathrm{H}(10)$ & 3337 & 8842 & 6055 & 27 \\
\hline $\mathrm{H}(12)$ & 3003 & 6842 & 4716 & 29 \\
\hline $\mathrm{H}(13)$ & 2795 & 6777 & 2381 & 35 \\
\hline $\mathrm{H}(15)$ & 1608 & 8443 & 2405 & 33 \\
\hline $\mathrm{H}(16)$ & 1779 & 8508 & 4751 & 26 \\
\hline $\mathrm{H}(17 \mathrm{~A})$ & 2302 & 7939 & 201 & 76 \\
\hline $\mathrm{H}(17 \mathrm{~B})$ & 1452 & 7544 & 432 & 76 \\
\hline $\mathrm{H}(17 \mathrm{C})$ & 2345 & 7194 & 284 & 76 \\
\hline $\mathrm{H}(21)$ & $3345(17)$ & $6345(12)$ & $7010(30)$ & 22 \\
\hline $\mathrm{H}(21 \mathrm{~A})$ & 3107 & 5538 & 2141 & 43 \\
\hline $\mathrm{H}(21 \mathrm{~B})$ & 2870 & 4840 & 1558 & 43 \\
\hline $\mathrm{H}(22)$ & 3189 & 4420 & 3557 & 30 \\
\hline $\mathrm{H}(23 \mathrm{~A})$ & 3568 & 5666 & 4426 & 28 \\
\hline $\mathrm{H}(23 \mathrm{~B})$ & 4132 & 5084 & 4890 & 28 \\
\hline $\mathrm{H}(24)$ & 2932 & 4677 & 6103 & 23 \\
\hline $\mathrm{H}(24 \mathrm{~A})$ & 2944 & 4674 & 6057 & 23 \\
\hline $\mathrm{H}(25 \mathrm{~A})$ & 2047 & 5823 & 6036 & 27 \\
\hline $\mathrm{H}(25 \mathrm{~B})$ & 1961 & 5401 & 4704 & 27 \\
\hline
\end{tabular}




\begin{tabular}{|c|c|c|c|c|}
\hline $\mathrm{H}(26 \mathrm{~A})$ & 824 & 5148 & 6050 & 32 \\
\hline $\mathrm{H}(26 \mathrm{~B})$ & 1452 & 4563 & 6108 & 32 \\
\hline $\mathrm{H}(27 \mathrm{~A})$ & 1160 & 5446 & 8210 & 33 \\
\hline $\mathrm{H}(27 \mathrm{~B})$ & 961 & 4718 & 8267 & 33 \\
\hline $\mathrm{H}(28 \mathrm{~A})$ & 2465 & 4526 & 8332 & 35 \\
\hline $\mathrm{H}(28 \mathrm{~B})$ & 2099 & 4868 & 9651 & 35 \\
\hline $\mathrm{H}(29 \mathrm{~A})$ & 2546 & 5849 & 8484 & 30 \\
\hline $\mathrm{H}(29 \mathrm{~B})$ & 3243 & 5450 & 9273 & 30 \\
\hline $\mathrm{H}(30)$ & 3925 & 5177 & 7407 & 23 \\
\hline $\mathrm{H}(25 \mathrm{C})$ & 1924 & 5293 & 4840 & 27 \\
\hline $\mathrm{H}(25 \mathrm{D})$ & 2072 & 5790 & 6025 & 27 \\
\hline $\mathrm{H}(26 \mathrm{C})$ & 891 & 5045 & 6346 & 43 \\
\hline $\mathrm{H}(26 \mathrm{D})$ & 1603 & 4534 & 6659 & 43 \\
\hline $\mathrm{H}(27 \mathrm{C})$ & 961 & 5431 & 8357 & 58 \\
\hline $\mathrm{H}(27 \mathrm{D})$ & 1442 & 4803 & 8749 & 58 \\
\hline $\mathrm{H}(28 \mathrm{C})$ & 2138 & 5635 & 9688 & 47 \\
\hline $\mathrm{H}(28 \mathrm{D})$ & 2220 & 5968 & 8245 & 47 \\
\hline $\mathrm{H}(29 \mathrm{C})$ & 3058 & 4813 & 8685 & 35 \\
\hline $\mathrm{H}(29 \mathrm{D})$ & 3490 & 5441 & 9211 & 35 \\
\hline $\mathrm{H}(30 \mathrm{~A})$ & 4067 & 5130 & 7097 & 23 \\
\hline $\mathrm{H}(32)$ & 5433 & 5540 & 9131 & 30 \\
\hline $\mathrm{H}(33)$ & 5509 & 5585 & 11484 & 35 \\
\hline $\mathrm{H}(35)$ & 4312 & 7249 & 11444 & 29 \\
\hline $\mathrm{H}(36)$ & 4194 & 7193 & 9091 & 26 \\
\hline $\mathrm{H}(37 \mathrm{~A})$ & 4513 & 6690 & 13601 & 41 \\
\hline $\mathrm{H}(37 \mathrm{~B})$ & 5526 & 6670 & 13475 & 41 \\
\hline $\mathrm{H}(37 \mathrm{C})$ & 5000 & 6035 & 13613 & 41 \\
\hline
\end{tabular}

Table S6. Hydrogen bonds for (-)-9 in $P 2_{12} 2$ [ $^{(}$and ${ }^{\circ}$ ].

\begin{tabular}{lcccc}
\hline $\mathrm{D}-\mathrm{H} \ldots \mathrm{A}$ & $\mathrm{d}(\mathrm{D}-\mathrm{H})$ & $\mathrm{d}(\mathrm{H} \ldots \mathrm{A})$ & $\mathrm{d}(\mathrm{D} \ldots \mathrm{A})$ & $<(\mathrm{DHA})$ \\
\hline $\mathrm{N}(1)-\mathrm{H}(1) \ldots \mathrm{O}(22)$ & $0.84(2)$ & $2.09(2)$ & $2.913(3)$ & $168(3)$ \\
$\mathrm{N}(21)-\mathrm{H}(21) \ldots \mathrm{O}(2)$ & $0.85(2)$ & $2.06(2)$ & $2.897(3)$ & $171(3)$ \\
& & & & \\
\hline
\end{tabular}




\section{$(-)-9$ in $P 4_{3} 2_{1} 2$}

The structure of the second crystals was solved in the tetragonal space group $P 4_{3} 2{ }_{1} 2$. The asymmetric unit contains one molecule of (-)-9, connected via hydrogen bonds to a symmetry equivalent. The absolute structure was confirmed with a Parsons parameter of -0.006(4).

Table S7. Crystal data and structure refinement for (-)-9 in $\mathbf{P 4} \mathbf{2}_{12}$.

\section{CCDC No}

Empirical formula

Formula weight

Temperature

Wavelength

Crystal system

Space group

Unit cell dimensions

Volume

Z

Density (calculated)

Absorption coefficient

$F(000)$

Crystal size

Theta range for data collection

Index ranges

Reflections collected

Independent reflections

Completeness to theta $=67.679^{\circ}$

Absorption correction

Refinement method

Data / restraints / parameters

Goodness-of-fit on $F^{2}$

Final R indices $[\mathrm{I}>2 \sigma(\mathrm{I})]$

$\mathrm{R}$ indices (all data)

Absolute structure parameter

Largest diff. peak and hole
2041506

$\mathrm{C}_{17} \mathrm{H}_{25} \mathrm{~N} \mathrm{O}_{2} \mathrm{~S}$

307.44

100(2) K

$1.54178 \AA$

Tetragonal

$P_{3} 2{ }_{12}$

$\mathrm{a}=8.4876(2) \AA$

$\alpha=90^{\circ}$.

$\mathrm{b}=8.4876(2) \AA$

$\beta=90^{\circ}$.

$\mathrm{c}=45.5241(16) \AA$

$\gamma=90^{\circ}$.

$3279.5(2) \AA^{3}$

8

\section{$1.245 \mathrm{Mg} / \mathrm{m}^{3}$}

$1.780 \mathrm{~mm}^{-1}$

1328

$0.193 \times 0.170 \times 0.121 \mathrm{~mm}^{3}$

3.884 to $74.484^{\circ}$.

$-10 \leq \mathrm{h} \leq 10,-10 \leq \mathrm{k} \leq 9,-56 \leq 1 \leq 56$

40739

$3359[\mathrm{R}(\mathrm{int})=0.0458]$

$99.9 \%$

Semi-empirical from equivalents

Full-matrix least-squares on $F^{2}$

3359 / 1 / 194

1.059

$\mathrm{R} 1=0.0270, \mathrm{wR} 2=0.0727$

$\mathrm{R} 1=0.0273, \mathrm{wR} 2=0.0730$

$-0.006(4)$

0.237 and -0.264 e. $\AA^{-3}$ 
Table S8. Atomic coordinates (x 104) and equivalent isotropic displacement parameters $\left(\AA^{2} \times 10^{3}\right)$ for (-)-9 in $\boldsymbol{P 4}_{3} \mathbf{2}_{1} \mathbf{2}$. $\mathrm{U}(\mathrm{eq})$ is defined as one third of the trace of the orthogonalized $\mathrm{U}^{\mathrm{ij}}$ tensor.

\begin{tabular}{lrccc}
\hline & $\mathrm{x}$ & $\mathrm{y}$ & $\mathrm{z}$ & $\mathrm{U}(\mathrm{eq})$ \\
\hline $\mathrm{S}(1)$ & & & & \\
$\mathrm{O}(1)$ & $5766(1)$ & $6938(1)$ & $5457(1)$ & $21(1)$ \\
$\mathrm{O}(2)$ & $4567(2)$ & $7063(2)$ & $5677(1)$ & $28(1)$ \\
$\mathrm{N}(1)$ & $5539(2)$ & $7700(1)$ & $5178(1)$ & $26(1)$ \\
$\mathrm{C}(1)$ & $6045(2)$ & $5097(2)$ & $5386(1)$ & $20(1)$ \\
$\mathrm{C}(2)$ & $10251(3)$ & $4459(2)$ & $6016(1)$ & $38(1)$ \\
$\mathrm{C}(3)$ & $9464(2)$ & $3255(2)$ & $5908(1)$ & $29(1)$ \\
$\mathrm{C}(4)$ & $9057(2)$ & $3028(2)$ & $5590(1)$ & $26(1)$ \\
$\mathrm{C}(5)$ & $7318(2)$ & $2580(2)$ & $5539(1)$ & $22(1)$ \\
$\mathrm{C}(6)$ & $7141(2)$ & $1942(2)$ & $5224(1)$ & $25(1)$ \\
$\mathrm{C}(7)$ & $5471(2)$ & $1767(2)$ & $5101(1)$ & $29(1)$ \\
$\mathrm{C}(8)$ & $4194(2)$ & $1266(2)$ & $5318(1)$ & $34(1)$ \\
$\mathrm{C}(9)$ & $3510(2)$ & $2636(2)$ & $5494(1)$ & $30(1)$ \\
$\mathrm{C}(10)$ & $4575(2)$ & $3338(2)$ & $5728(1)$ & $29(1)$ \\
$\mathrm{C}(11)$ & $6194(2)$ & $3930(2)$ & $5627(1)$ & $22(1)$ \\
$\mathrm{C}(12)$ & $7519(2)$ & $7700(2)$ & $5610(1)$ & $22(1)$ \\
$\mathrm{C}(13)$ & $7535(2)$ & $8190(2)$ & $5902(1)$ & $25(1)$ \\
$\mathrm{C}(14)$ & $8921(2)$ & $8766(2)$ & $6022(1)$ & $27(1)$ \\
$\mathrm{C}(15)$ & $10299(2)$ & $8867(2)$ & $5858(1)$ & $25(1)$ \\
$\mathrm{C}(16)$ & $10257(2)$ & $8364(2)$ & $5564(1)$ & $26(1)$ \\
$\mathrm{C}(17)$ & $8884(2)$ & $7783(2)$ & $5441(1)$ & $25(1)$ \\
& $11794(2)$ & $9506(2)$ & $5989(1)$ & $30(1)$ \\
\hline & & & & \\
\hline
\end{tabular}


Table S9. Bond lengths $[\AA]$ and angles $\left[{ }^{\circ}\right]$ for (-)-9 in $\mathbf{P 4}_{3} \mathbf{2}_{1} \mathbf{2}$.

\begin{tabular}{|c|c|c|c|}
\hline $\mathrm{S}(1)-\mathrm{O}(1)$ & $1.4329(13)$ & $\mathrm{C}(13)-\mathrm{H}(13)$ & 0.9500 \\
\hline $\mathrm{S}(1)-\mathrm{O}(2)$ & $1.4391(12)$ & $C(14)-C(15)$ & $1.405(2)$ \\
\hline $\mathrm{S}(1)-\mathrm{N}(1)$ & $1.6122(14)$ & $C(14)-C(17)$ & $1.504(3)$ \\
\hline $\mathrm{S}(1)-\mathrm{C}(11)$ & $1.7658(18)$ & $C(15)-C(16)$ & $1.383(3)$ \\
\hline $\mathrm{N}(1)-\mathrm{C}(10)$ & $1.482(2)$ & $\mathrm{C}(15)-\mathrm{H}(15)$ & 0.9500 \\
\hline $\mathrm{N}(1)-\mathrm{H}(1)$ & $0.867(17)$ & $\mathrm{C}(16)-\mathrm{H}(16)$ & 0.9500 \\
\hline$C(1)-C(2)$ & $1.317(3)$ & $\mathrm{C}(17)-\mathrm{H}(17 \mathrm{~A})$ & 0.9800 \\
\hline $\mathrm{C}(1)-\mathrm{H}(1 \mathrm{~A})$ & 0.9500 & $\mathrm{C}(17)-\mathrm{H}(17 \mathrm{~B})$ & 0.9800 \\
\hline C(1)-H(1B) & 0.9500 & $\mathrm{C}(17)-\mathrm{H}(17 \mathrm{C})$ & 0.9800 \\
\hline $\mathrm{C}(2)-\mathrm{C}(3)$ & $1.498(2)$ & & \\
\hline $\mathrm{C}(2)-\mathrm{H}(2)$ & 0.9500 & $\mathrm{O}(1)-\mathrm{S}(1)-\mathrm{O}(2)$ & $119.27(8)$ \\
\hline $\mathrm{C}(3)-\mathrm{C}(4)$ & $1.542(2)$ & $\mathrm{O}(1)-\mathrm{S}(1)-\mathrm{N}(1)$ & $108.37(8)$ \\
\hline $\mathrm{C}(3)-\mathrm{H}(3 \mathrm{~A})$ & 0.9900 & $\mathrm{O}(2)-\mathrm{S}(1)-\mathrm{N}(1)$ & $106.30(7)$ \\
\hline $\mathrm{C}(3)-\mathrm{H}(3 \mathrm{AB})$ & 0.9900 & $\mathrm{O}(1)-\mathrm{S}(1)-\mathrm{C}(11)$ & $107.13(8)$ \\
\hline$C(4)-C(5)$ & $1.541(2)$ & $\mathrm{O}(2)-\mathrm{S}(1)-\mathrm{C}(11)$ & $107.31(8)$ \\
\hline$C(4)-C(10)$ & $1.544(2)$ & $\mathrm{N}(1)-\mathrm{S}(1)-\mathrm{C}(11)$ & $108.04(8)$ \\
\hline $\mathrm{C}(4)-\mathrm{H}(4)$ & 1.0000 & $\mathrm{C}(10)-\mathrm{N}(1)-\mathrm{S}(1)$ & $120.89(11)$ \\
\hline$C(5)-C(6)$ & $1.531(3)$ & $\mathrm{C}(10)-\mathrm{N}(1)-\mathrm{H}(1)$ & $117.4(14)$ \\
\hline $\mathrm{C}(5)-\mathrm{H}(5 \mathrm{~A})$ & 0.9900 & $\mathrm{~S}(1)-\mathrm{N}(1)-\mathrm{H}(1)$ & $112.8(15)$ \\
\hline $\mathrm{C}(5)-\mathrm{H}(5 \mathrm{AB})$ & 0.9900 & $\mathrm{C}(2)-\mathrm{C}(1)-\mathrm{H}(1 \mathrm{~A})$ & 120.0 \\
\hline$C(6)-C(7)$ & $1.527(3)$ & $\mathrm{C}(2)-\mathrm{C}(1)-\mathrm{H}(1 \mathrm{~B})$ & 120.0 \\
\hline $\mathrm{C}(6)-\mathrm{H}(6 \mathrm{~A})$ & 0.9900 & $\mathrm{H}(1 \mathrm{~A})-\mathrm{C}(1)-\mathrm{H}(1 \mathrm{~B})$ & 120.0 \\
\hline $\mathrm{C}(6)-\mathrm{H}(6 \mathrm{AB})$ & 0.9900 & $\mathrm{C}(1)-\mathrm{C}(2)-\mathrm{C}(3)$ & $125.35(18)$ \\
\hline$C(7)-C(8)$ & $1.527(3)$ & $\mathrm{C}(1)-\mathrm{C}(2)-\mathrm{H}(2)$ & 117.3 \\
\hline $\mathrm{C}(7)-\mathrm{H}(7 \mathrm{~A})$ & 0.9900 & $\mathrm{C}(3)-\mathrm{C}(2)-\mathrm{H}(2)$ & 117.3 \\
\hline $\mathrm{C}(7)-\mathrm{H}(7 \mathrm{AB})$ & 0.9900 & $C(2)-C(3)-C(4)$ & $113.48(15)$ \\
\hline $\mathrm{C}(8)-\mathrm{C}(9)$ & $1.520(3)$ & $\mathrm{C}(2)-\mathrm{C}(3)-\mathrm{H}(3 \mathrm{~A})$ & 108.9 \\
\hline $\mathrm{C}(8)-\mathrm{H}(8 \mathrm{~A})$ & 0.9900 & $\mathrm{C}(4)-\mathrm{C}(3)-\mathrm{H}(3 \mathrm{~A})$ & 108.9 \\
\hline $\mathrm{C}(8)-\mathrm{H}(8 \mathrm{AB})$ & 0.9900 & $\mathrm{C}(2)-\mathrm{C}(3)-\mathrm{H}(3 \mathrm{AB})$ & 108.9 \\
\hline$C(9)-C(10)$ & $1.534(2)$ & $\mathrm{C}(4)-\mathrm{C}(3)-\mathrm{H}(3 \mathrm{AB})$ & 108.9 \\
\hline $\mathrm{C}(9)-\mathrm{H}(9 \mathrm{~A})$ & 0.9900 & $\mathrm{H}(3 \mathrm{~A})-\mathrm{C}(3)-\mathrm{H}(3 \mathrm{AB})$ & 107.7 \\
\hline $\mathrm{C}(9)-\mathrm{H}(9 \mathrm{AB})$ & 0.9900 & $\mathrm{C}(5)-\mathrm{C}(4)-\mathrm{C}(3)$ & $108.71(14)$ \\
\hline $\mathrm{C}(10)-\mathrm{H}(10)$ & 1.0000 & $C(5)-C(4)-C(10)$ & $116.26(14)$ \\
\hline$C(11)-C(16)$ & $1.393(2)$ & $C(3)-C(4)-C(10)$ & $111.64(14)$ \\
\hline $\mathrm{C}(11)-\mathrm{C}(12)$ & $1.394(2)$ & $\mathrm{C}(5)-\mathrm{C}(4)-\mathrm{H}(4)$ & 106.6 \\
\hline$C(12)-C(13)$ & $1.386(3)$ & $\mathrm{C}(3)-\mathrm{C}(4)-\mathrm{H}(4)$ & 106.6 \\
\hline $\mathrm{C}(12)-\mathrm{H}(12)$ & 0.9500 & $\mathrm{C}(10)-\mathrm{C}(4)-\mathrm{H}(4)$ & 106.6 \\
\hline $\mathrm{C}(13)-\mathrm{C}(14)$ & $1.392(3)$ & $\mathrm{C}(6)-\mathrm{C}(5)-\mathrm{C}(4)$ & $117.76(15)$ \\
\hline
\end{tabular}




\begin{tabular}{|c|c|c|c|}
\hline $\mathrm{C}(6)-\mathrm{C}(5)-\mathrm{H}(5 \mathrm{~A})$ & 107.9 & $\mathrm{C}(11)-\mathrm{C}(12)-\mathrm{H}(12)$ & 120.3 \\
\hline $\mathrm{C}(4)-\mathrm{C}(5)-\mathrm{H}(5 \mathrm{~A})$ & 107.9 & $\mathrm{C}(12)-\mathrm{C}(13)-\mathrm{C}(14)$ & $121.56(15)$ \\
\hline $\mathrm{C}(6)-\mathrm{C}(5)-\mathrm{H}(5 \mathrm{AB})$ & 107.9 & $\mathrm{C}(12)-\mathrm{C}(13)-\mathrm{H}(13)$ & 119.2 \\
\hline $\mathrm{C}(4)-\mathrm{C}(5)-\mathrm{H}(5 \mathrm{AB})$ & 107.9 & $\mathrm{C}(14)-\mathrm{C}(13)-\mathrm{H}(13)$ & 119.2 \\
\hline $\mathrm{H}(5 \mathrm{~A})-\mathrm{C}(5)-\mathrm{H}(5 \mathrm{AB})$ & 107.2 & $\mathrm{C}(13)-\mathrm{C}(14)-\mathrm{C}(15)$ & $118.18(17)$ \\
\hline$C(7)-C(6)-C(5)$ & $116.55(15)$ & $\mathrm{C}(13)-\mathrm{C}(14)-\mathrm{C}(17)$ & $121.17(15)$ \\
\hline $\mathrm{C}(7)-\mathrm{C}(6)-\mathrm{H}(6 \mathrm{~A})$ & 108.2 & $\mathrm{C}(15)-\mathrm{C}(14)-\mathrm{C}(17)$ & $120.64(16)$ \\
\hline $\mathrm{C}(5)-\mathrm{C}(6)-\mathrm{H}(6 \mathrm{~A})$ & 108.2 & $C(16)-C(15)-C(14)$ & $120.94(17)$ \\
\hline $\mathrm{C}(7)-\mathrm{C}(6)-\mathrm{H}(6 \mathrm{AB})$ & 108.2 & $\mathrm{C}(16)-\mathrm{C}(15)-\mathrm{H}(15)$ & 119.5 \\
\hline $\mathrm{C}(5)-\mathrm{C}(6)-\mathrm{H}(6 \mathrm{AB})$ & 108.2 & $\mathrm{C}(14)-\mathrm{C}(15)-\mathrm{H}(15)$ & 119.5 \\
\hline $\mathrm{H}(6 \mathrm{~A})-\mathrm{C}(6)-\mathrm{H}(6 \mathrm{AB})$ & 107.3 & $\mathrm{C}(15)-\mathrm{C}(16)-\mathrm{C}(11)$ & $119.74(15)$ \\
\hline$C(6)-C(7)-C(8)$ & $113.44(16)$ & $\mathrm{C}(15)-\mathrm{C}(16)-\mathrm{H}(16)$ & 120.1 \\
\hline $\mathrm{C}(6)-\mathrm{C}(7)-\mathrm{H}(7 \mathrm{~A})$ & 108.9 & $\mathrm{C}(11)-\mathrm{C}(16)-\mathrm{H}(16)$ & 120.1 \\
\hline $\mathrm{C}(8)-\mathrm{C}(7)-\mathrm{H}(7 \mathrm{~A})$ & 108.9 & $\mathrm{C}(14)-\mathrm{C}(17)-\mathrm{H}(17 \mathrm{~A})$ & 109.5 \\
\hline $\mathrm{C}(6)-\mathrm{C}(7)-\mathrm{H}(7 \mathrm{AB})$ & 108.9 & $\mathrm{C}(14)-\mathrm{C}(17)-\mathrm{H}(17 \mathrm{~B})$ & 109.5 \\
\hline $\mathrm{C}(8)-\mathrm{C}(7)-\mathrm{H}(7 \mathrm{AB})$ & 108.9 & $\mathrm{H}(17 \mathrm{~A})-\mathrm{C}(17)-\mathrm{H}(17 \mathrm{~B})$ & 109.5 \\
\hline $\mathrm{H}(7 \mathrm{~A})-\mathrm{C}(7)-\mathrm{H}(7 \mathrm{AB})$ & 107.7 & $\mathrm{C}(14)-\mathrm{C}(17)-\mathrm{H}(17 \mathrm{C})$ & 109.5 \\
\hline $\mathrm{C}(9)-\mathrm{C}(8)-\mathrm{C}(7)$ & $116.22(16)$ & $\mathrm{H}(17 \mathrm{~A})-\mathrm{C}(17)-\mathrm{H}(17 \mathrm{C})$ & 109.5 \\
\hline $\mathrm{C}(9)-\mathrm{C}(8)-\mathrm{H}(8 \mathrm{~A})$ & 108.2 & $\mathrm{H}(17 \mathrm{~B})-\mathrm{C}(17)-\mathrm{H}(17 \mathrm{C})$ & 109.5 \\
\hline $\mathrm{C}(7)-\mathrm{C}(8)-\mathrm{H}(8 \mathrm{~A})$ & 108.2 & & \\
\hline $\mathrm{C}(9)-\mathrm{C}(8)-\mathrm{H}(8 \mathrm{AB})$ & 108.2 & & \\
\hline $\mathrm{C}(7)-\mathrm{C}(8)-\mathrm{H}(8 \mathrm{AB})$ & 108.2 & & \\
\hline $\mathrm{H}(8 \mathrm{~A})-\mathrm{C}(8)-\mathrm{H}(8 \mathrm{AB})$ & 107.4 & & \\
\hline $\mathrm{C}(8)-\mathrm{C}(9)-\mathrm{C}(10)$ & $116.73(14)$ & & \\
\hline $\mathrm{C}(8)-\mathrm{C}(9)-\mathrm{H}(9 \mathrm{~A})$ & 108.1 & & \\
\hline $\mathrm{C}(10)-\mathrm{C}(9)-\mathrm{H}(9 \mathrm{~A})$ & 108.1 & & \\
\hline $\mathrm{C}(8)-\mathrm{C}(9)-\mathrm{H}(9 \mathrm{AB})$ & 108.1 & & \\
\hline $\mathrm{C}(10)-\mathrm{C}(9)-\mathrm{H}(9 \mathrm{AB})$ & 108.1 & & \\
\hline $\mathrm{H}(9 \mathrm{~A})-\mathrm{C}(9)-\mathrm{H}(9 \mathrm{AB})$ & 107.3 & & \\
\hline $\mathrm{N}(1)-\mathrm{C}(10)-\mathrm{C}(9)$ & $111.39(13)$ & & \\
\hline $\mathrm{N}(1)-\mathrm{C}(10)-\mathrm{C}(4)$ & $110.87(13)$ & & \\
\hline $\mathrm{C}(9)-\mathrm{C}(10)-\mathrm{C}(4)$ & $112.84(15)$ & & \\
\hline $\mathrm{N}(1)-\mathrm{C}(10)-\mathrm{H}(10)$ & 107.1 & & \\
\hline $\mathrm{C}(9)-\mathrm{C}(10)-\mathrm{H}(10)$ & 107.1 & & \\
\hline $\mathrm{C}(4)-\mathrm{C}(10)-\mathrm{H}(10)$ & 107.1 & & \\
\hline $\mathrm{C}(16)-\mathrm{C}(11)-\mathrm{C}(12)$ & $120.26(17)$ & & \\
\hline $\mathrm{C}(16)-\mathrm{C}(11)-\mathrm{S}(1)$ & $120.06(13)$ & & \\
\hline $\mathrm{C}(12)-\mathrm{C}(11)-\mathrm{S}(1)$ & $119.67(14)$ & & \\
\hline $\mathrm{C}(13)-\mathrm{C}(12)-\mathrm{C}(11)$ & $119.31(16)$ & & \\
\hline $\mathrm{C}(13)-\mathrm{C}(12)-\mathrm{H}(12)$ & 120.3 & & \\
\hline
\end{tabular}


Table S10. Anisotropic displacement parameters $\left(\AA^{2} \times 10^{3}\right)$ for $(-)-\mathbf{9}$ in $\mathbf{P 4}_{3} \mathbf{2}_{1} \mathbf{2}$. The anisotropic displacement factor exponent takes the form: $-2 p^{2}\left[h^{2} a^{* 2} U^{11}+\ldots+2 h k a^{*} b^{*} U^{12}\right]$

\begin{tabular}{|c|c|c|c|c|c|c|}
\hline & $\mathrm{U}^{11}$ & $\mathrm{U}^{22}$ & $\mathrm{U}^{33}$ & $\mathrm{U}^{23}$ & $\mathrm{U}^{13}$ & $\mathrm{U}^{12}$ \\
\hline $\mathrm{S}(1)$ & $27(1)$ & $20(1)$ & $17(1)$ & $-2(1)$ & 1(1) & $5(1)$ \\
\hline $\mathrm{O}(1)$ & $32(1)$ & $28(1)$ & $24(1)$ & $-4(1)$ & $6(1)$ & $8(1)$ \\
\hline $\mathrm{O}(2)$ & $36(1)$ & $23(1)$ & $19(1)$ & $0(1)$ & $-3(1)$ & $6(1)$ \\
\hline $\mathrm{N}(1)$ & $27(1)$ & $20(1)$ & $15(1)$ & $-1(1)$ & $3(1)$ & $3(1)$ \\
\hline $\mathrm{C}(1)$ & $48(1)$ & $31(1)$ & $35(1)$ & $-2(1)$ & $-11(1)$ & $1(1)$ \\
\hline $\mathrm{C}(2)$ & $32(1)$ & $28(1)$ & $28(1)$ & $3(1)$ & $-6(1)$ & $4(1)$ \\
\hline$C(3)$ & $26(1)$ & $28(1)$ & $25(1)$ & $-1(1)$ & $0(1)$ & $4(1)$ \\
\hline $\mathrm{C}(4)$ & $27(1)$ & $22(1)$ & $19(1)$ & $1(1)$ & $-1(1)$ & $4(1)$ \\
\hline $\mathrm{C}(5)$ & $31(1)$ & $22(1)$ & $22(1)$ & $-3(1)$ & $0(1)$ & $5(1)$ \\
\hline $\mathrm{C}(6)$ & $35(1)$ & $26(1)$ & $26(1)$ & $-5(1)$ & $-5(1)$ & $2(1)$ \\
\hline$C(7)$ & $33(1)$ & $26(1)$ & $42(1)$ & $1(1)$ & $-4(1)$ & $-3(1)$ \\
\hline $\mathrm{C}(8)$ & $26(1)$ & $27(1)$ & $36(1)$ & 10(1) & $3(1)$ & $1(1)$ \\
\hline $\mathrm{C}(9)$ & $31(1)$ & $31(1)$ & $24(1)$ & $7(1)$ & $8(1)$ & $7(1)$ \\
\hline $\mathrm{C}(10)$ & $28(1)$ & $24(1)$ & $15(1)$ & $0(1)$ & $0(1)$ & $4(1)$ \\
\hline $\mathrm{C}(11)$ & $31(1)$ & $19(1)$ & $17(1)$ & $0(1)$ & $-1(1)$ & $3(1)$ \\
\hline $\mathrm{C}(12)$ & $36(1)$ & $22(1)$ & $17(1)$ & $-1(1)$ & $5(1)$ & $3(1)$ \\
\hline $\mathrm{C}(13)$ & $43(1)$ & $23(1)$ & $16(1)$ & $-2(1)$ & $-1(1)$ & $1(1)$ \\
\hline C(14) & $36(1)$ & $18(1)$ & $20(1)$ & $1(1)$ & $-2(1)$ & $3(1)$ \\
\hline $\mathrm{C}(15)$ & $33(1)$ & $26(1)$ & $19(1)$ & $0(1)$ & $2(1)$ & $3(1)$ \\
\hline$C(16)$ & $36(1)$ & $25(1)$ & $14(1)$ & $-2(1)$ & 2(1) & $4(1)$ \\
\hline $\mathrm{C}(17)$ & $37(1)$ & $28(1)$ & $24(1)$ & $-1(1)$ & $-4(1)$ & $2(1)$ \\
\hline
\end{tabular}


Table S11. Hydrogen coordinates (x $\left.10^{4}\right)$ and isotropic displacement parameters $\left(\AA^{2} \times 10^{3}\right)$ for $(-)-\mathbf{9}$ in $\mathbf{P 4}_{3} \mathbf{2}_{1} \mathbf{2}$.

\begin{tabular}{|c|c|c|c|c|}
\hline & $\mathrm{x}$ & $\mathrm{y}$ & $\mathrm{z}$ & $\mathrm{U}(\mathrm{eq})$ \\
\hline $\mathrm{H}(1)$ & $6610(20)$ & $4950(30)$ & $5230(4)$ & 24 \\
\hline $\mathrm{H}(1 \mathrm{~A})$ & 10609 & 5275 & 5890 & 45 \\
\hline $\mathrm{H}(1 \mathrm{~B})$ & 10461 & 4513 & 6221 & 45 \\
\hline $\mathrm{H}(2)$ & 9128 & 2465 & 6041 & 35 \\
\hline $\mathrm{H}(3 \mathrm{~A})$ & 9737 & 2190 & 5508 & 32 \\
\hline $\mathrm{H}(3 \mathrm{AB})$ & 9288 & 4015 & 5482 & 32 \\
\hline $\mathrm{H}(4)$ & 7085 & 1680 & 5674 & 27 \\
\hline $\mathrm{H}(5 \mathrm{~A})$ & 7736 & 2648 & 5091 & 30 \\
\hline $\mathrm{H}(5 \mathrm{AB})$ & 7654 & 896 & 5216 & 30 \\
\hline $\mathrm{H}(6 \mathrm{~A})$ & 5156 & 2788 & 5013 & 35 \\
\hline $\mathrm{H}(6 \mathrm{AB})$ & 5503 & 984 & 4940 & 35 \\
\hline $\mathrm{H}(7 \mathrm{~A})$ & 4642 & 484 & 5455 & 41 \\
\hline $\mathrm{H}(7 \mathrm{AB})$ & 3331 & 745 & 5208 & 41 \\
\hline $\mathrm{H}(8 \mathrm{~A})$ & 3218 & 3484 & 5355 & 36 \\
\hline $\mathrm{H}(8 \mathrm{AB})$ & 2529 & 2271 & 5590 & 36 \\
\hline $\mathrm{H}(9 \mathrm{~A})$ & 4742 & 2531 & 5882 & 34 \\
\hline $\mathrm{H}(9 \mathrm{AB})$ & 4010 & 4230 & 5821 & 34 \\
\hline $\mathrm{H}(10)$ & 6685 & 4486 & 5798 & 27 \\
\hline $\mathrm{H}(12)$ & 6605 & 8130 & 6018 & 30 \\
\hline $\mathrm{H}(13)$ & 8930 & 9099 & 6221 & 32 \\
\hline $\mathrm{H}(15)$ & 11185 & 8423 & 5448 & 31 \\
\hline $\mathrm{H}(16)$ & 8872 & 7443 & 5242 & 30 \\
\hline $\mathrm{H}(17 \mathrm{~A})$ & 12703 & 9062 & 5885 & 45 \\
\hline $\mathrm{H}(17 \mathrm{~B})$ & 11848 & 9215 & 6197 & 45 \\
\hline $\mathrm{H}(17 \mathrm{C})$ & 11806 & 10656 & 5971 & 45 \\
\hline
\end{tabular}


Table S12. Hydrogen bonds for (-)-9 in $\mathbf{P 4}_{3} \mathbf{2}_{1} 2$ [ $\AA$ and ${ }^{\circ}$ ].

\begin{tabular}{lllll}
\hline D-H...A & d(D-H) & $d(H \ldots A)$ & $d(D \ldots A)$ & $<($ DHA $)$ \\
\hline $\mathrm{N}(1)-\mathrm{H}(1) \ldots \mathrm{O}(2) \# 1$ & $0.867(17)$ & $2.135(18)$ & $2.9511(18)$ & $157(2)$
\end{tabular}

Symmetry transformations used to generate equivalent atoms:

$\# 1 \mathrm{y}, \mathrm{x},-\mathrm{z}+1$ 


\section{Optical Rotatory Dispersion Experiments}

Table S13. Experimental specific rotation of $(3 \mathrm{a} R, 8 \mathrm{a} S)-d_{1}-\mathbf{1}$ and $(3 \mathrm{a} S, 8 \mathrm{a} R)-d_{1}-\mathbf{1}$ at different wavelengths. ${ }^{a}$

\begin{tabular}{|c|c|c|c|c|c|c|}
\hline \multirow{2}{*}{} & \multicolumn{3}{|c|}{$(3 \mathrm{a} R, 8 \mathrm{a} S)-d_{1}-1$} & \multicolumn{3}{c|}{$(3 \mathrm{a} S, 8 \mathrm{a} R)-d_{1}-1$} \\
\cline { 2 - 7 } & run 1 & run 2 & run 3 & run 1 & run 2 & run 3 \\
\hline $589 \mathrm{~nm}$ & $+0.3 \pm 0.4$ & $+0.5 \pm 1.1$ & $+0.1 \pm 0.1$ & $0.0 \pm 0.1$ & $0.0 \pm 0.1$ & $-0.1 \pm 0.1$ \\
\hline $546 \mathrm{~nm}$ & $+0.2 \pm 0.1$ & $+0.4 \pm 0.1$ & $+0.2 \pm 0.1$ & $-0.2 \pm 0.2$ & $-0.2 \pm 0.1$ & $-0.2 \pm 0.1$ \\
\hline $436 \mathrm{~nm}$ & $+0.2 \pm 0.4$ & $+0.2 \pm 0.1$ & $+0.3 \pm 0.3$ & $+0.2 \pm 0.2$ & $0.0 \pm 0.2$ & $-0.1 \pm 0.3$ \\
\hline $405 \mathrm{~nm}$ & $+0.2 \pm 0.3$ & $+0.3 \pm 0.2$ & $+0.1 \pm 0.3$ & $+0.1 \pm 0.1$ & $+0.1 \pm 0.2$ & $0.0 \pm 0.2$ \\
\hline $365 \mathrm{~nm}$ & $-0.3 \pm 0.7$ & $-0.3 \pm 0.3$ & $+0.1 \pm 0.9$ & $-0.2 \pm 0.7$ & $-0.3 \pm 0.5$ & $-0.1 \pm 0.4$ \\
\hline
\end{tabular}

${ }^{a}$ Measurement was repeated ten times in every run. Concentration: $0.5(\mathrm{~g} / 100 \mathrm{~mL})$. Solvent: $\mathrm{CDCl}_{3}$. 


\section{Vibrational Circular Dichroism Experiments}

VCD spectra in the spectral region of 1800 to $800 \mathrm{~cm}^{-1}$ were recorded with a Bruker PMA50 photoelastic modulator unit attached to a Bruker Vertex 70 spectrometer in conjunction with a Hinds PEM-100 controller and a Stanford Research Systems SR830 DSP lock-in amplifier (LIA). The LIA was set to a voltage of $1 \mathrm{mV}$ and the PEM was tuned to maximize its sensitivity in the spectral region around $1200 \mathrm{~cm}^{-1}$. The spectral resolution for IR and VCD spectra was $4 \mathrm{~cm}^{-1}$. The interferograms of up to 8 individual measurements of 2000 scans each were averaged prior to generation of the VCD spectrum. Sample VCD spectra were corrected by subtracting the VCD trace of the empty measurement cell from the sample VCD trace.

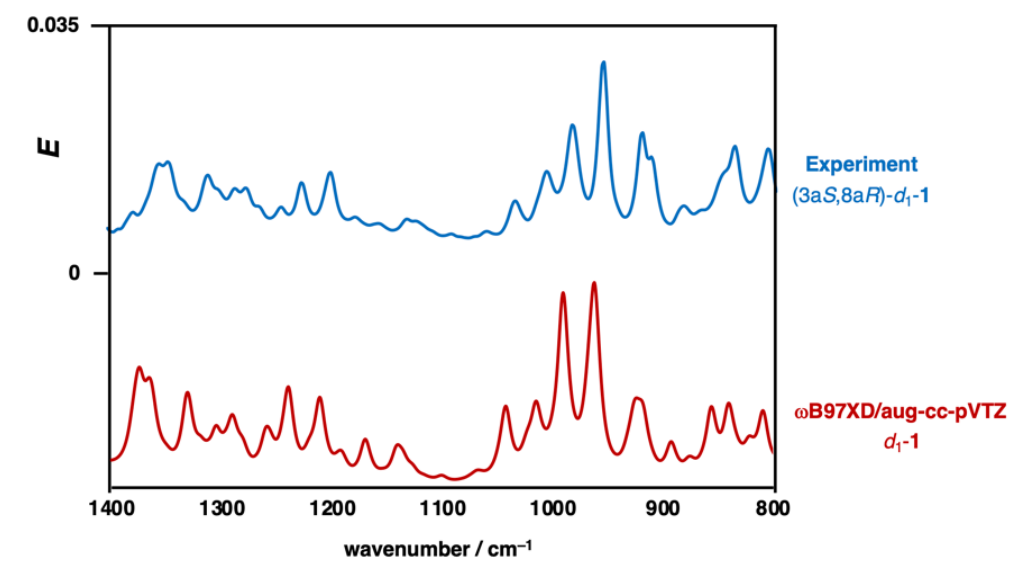

Figure S4. Measured (neat; blue) and simulated ( $\omega$ B97XD/aug-cc-pVTZ; gas phase, $6 \mathrm{~cm}^{-1}$ line width, scaled by 0.98 ; red) IR spectra of $(3 \mathrm{a} S, 8 \mathrm{a} R)-d_{1}-1$.

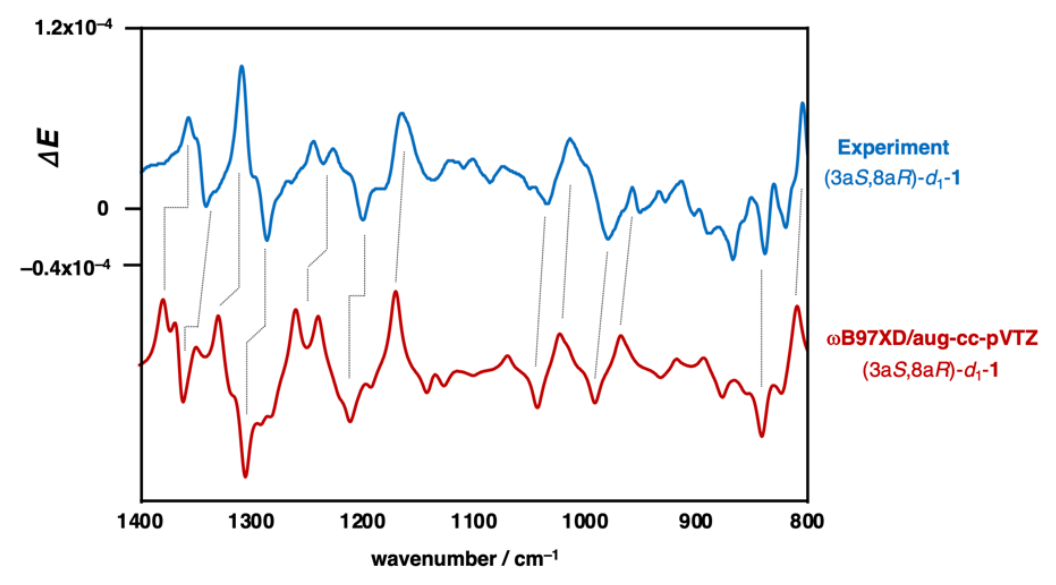

Figure S5. Measured (neat; blue) and simulated ( $\omega$ B97XD/aug-cc-pVTZ; gas phase, $6 \mathrm{~cm}^{-1}$ line width, scaled by 0.98 ; red) VCD spectra of $(3 \mathrm{a} S, 8 \mathrm{a} R)-d_{1}-1$. The spectral region from 1800 to $1400 \mathrm{~cm}^{-1}$ was devoid of any substrate signals. 


$$
\begin{gathered}
-0.527732000 \\
-0.1458860 \\
-2.005754000
\end{gathered}
$$

0.346610000

0.195112000

0.008797000

1.838364000

2.329669000

1.950148000

2.583941000

2. 614494000

3.621929000

2.000675000

2.702852000

1.931623000

$-2.514817000$

$-2.144728000$

$-2.550292000$

$-2.552047000$

$-1.712273000$

$-3.638419000$

$-2.228843000$

$$
\begin{aligned}
& -0.659197000 \\
& -0.919140000
\end{aligned}
$$

$-1.056161000$

$-1.400228000$

$-1.002481000$

$-2.438800000$

$-1.402934000$

$-2.191976000$

$-1.679814000$

$-0.096966000$

0.088258000

$-0.224492000$

1.140778000

1. 965156000

0.977622000

$-0.831989000$

$-2.118667000$

$-0.148447000$

1.805277000

1.783401000

$-0.098789000$

$-0.516372000$
0.618416000

1.608522000

0.511494000

$-0.401042000$

$-1.408850000$

$-0.433638000$

$-0.061547000$

$-0.635485000$

0.991875000

$-0.317416000$

$-1.396049000$

$-0.001216000$

0.361275000

0.222785000

1. 441557000

1.452731000

0.309506000

$-0.586971000$

0.348341000

$-1.187121000$

$-0.603512000$

\begin{tabular}{|c|c|c|c|}
\hline 1 & -0.509990000 & 2.632955000 & -0.084045000 \\
\hline 6 & -0.635615000 & 1.552758000 & -0.188753000 \\
\hline 1 & -0.617641000 & 1.357993000 & -1.265916000 \\
\hline 6 & 0.572301000 & 0.887983000 & 0.467894000 \\
\hline 6 & 1.893274000 & 1.203512000 & -0.286755000 \\
\hline $1($ I SO $=2)$ & 0.649791000 & 1.297742000 & 1.478102000 \\
\hline 6 & 0.527144000 & -0.657848000 & 0.618673000 \\
\hline 1 & 0.142853000 & -0.916925000 & 1.608105000 \\
\hline 6 & 2.005555000 & -1.053995000 & 0.515969000 \\
\hline 6 & -0.344447000 & -1.399200000 & -0.402661000 \\
\hline 1 & -0.192536000 & -0.999808000 & -1.409794000 \\
\hline 1 & -0.005198000 & -2.437323000 & -0.436313000 \\
\hline 6 & -1.836448000 & -1.404290000 & -0.064344000 \\
\hline 1 & -2.326548000 & -2.192476000 & -0.640501000 \\
\hline 1 & -1.948547000 & -1.683849000 & 0.988368000 \\
\hline 6 & -2.583338000 & -0.098536000 & -0.317554000 \\
\hline 1 & -2.614239000 & 0.088858000 & -1.395830000 \\
\hline 1 & -3.621144000 & -0.227920000 & -0.001484000 \\
\hline 6 & -2.001486000 & 1.138723000 & 0.363348000 \\
\hline 1 & -2.704901000 & 1.962367000 & 0.226617000 \\
\hline 1 & -1.931611000 & 0.973793000 & 1.443322000 \\
\hline 1 & 2.512183000 & -0.826674000 & 1.457754000 \\
\hline 1 & 2.145782000 & -2.117020000 & 0.317359000 \\
\hline 6 & 2.551742000 & -0.149143000 & -0.584144000 \\
\hline 1 & 2.548624000 & 1.811683000 & 0.337847000 \\
\hline 1 & 1.706770000 & 1.775791000 & -1.196178000 \\
\hline 1 & 3.639831000 & -0.097414000 & -0.597193000 \\
\hline 1 & 2.234430000 & -0.521558000 & -1.560262000 \\
\hline \multicolumn{4}{|c|}{$\begin{array}{l}\boldsymbol{E}=-391.939303 \text { a.u. } \\
V \boldsymbol{E}=164.44702 \mathrm{kcal} \mathrm{mol}^{-1}\end{array}$} \\
\hline
\end{tabular}

$-1.563465000$

$$
\begin{gathered}
\boldsymbol{E}=-391.939303 \mathrm{a.u} . \\
\boldsymbol{Z} \boldsymbol{P \boldsymbol { E } E}=164.45502 \mathrm{kcal} \mathrm{mol}^{-1} \\
\boldsymbol{G}_{\mathbf{2 9 8}}=-391.711676 \mathrm{a.u} .
\end{gathered}
$$

\section{Conformer III}

\section{Conformer IV}




\begin{tabular}{|c|c|c|c|}
\hline 6 & -0.566318000 & 1.443692000 & 0.576878000 \\
\hline 1 & -2.239438000 & 2.126741000 & -0.596708000 \\
\hline 1 & -2.695450000 & 1.395265000 & 0.921854000 \\
\hline 6 & 0.491131000 & 0.785578000 & -0.305459000 \\
\hline 1 & -0.330838000 & 2.507026000 & 0.664144000 \\
\hline 1 & -0.476632000 & 1.044671000 & 1.589346000 \\
\hline 1 & 0.327929000 & 1.121525000 & -1.333189000 \\
\hline 6 & 1.917926000 & 1.186129000 & 0.104599000 \\
\hline 6 & -0.566323000 & -1.443690000 & 0.576878000 \\
\hline 1 & -1.749204000 & 0.000006000 & -1.614021000 \\
\hline 1 & -2.239430000 & -2.126737000 & -0.596727000 \\
\hline 1 & -0.330842000 & -2.507024000 & 0.664150000 \\
\hline 1 & -0.476643000 & -1.044665000 & 1.589345000 \\
\hline 6 & 1.917924000 & -1.186128000 & 0.104609000 \\
\hline $1($ I SO $=2)$ & 0.327933000 & -1.121530000 & -1.333186000 \\
\hline 1 & 1.962018000 & 1.314002000 & 1.190843000 \\
\hline 1 & 2.228103000 & 2.129928000 & -0.344001000 \\
\hline 6 & 2.787761000 & -0.000002000 & -0.295974000 \\
\hline 1 & 3.765626000 & -0.000001000 & 0.184748000 \\
\hline 1 & 2.950046000 & -0.000007000 & -1.377129000 \\
\hline 1 & 2.228100000 & -2.129932000 & -0.343982000 \\
\hline 1 & 1.962013000 & -1.313991000 & 1.190854000 \\
\hline \multicolumn{4}{|c|}{$\begin{array}{c}\boldsymbol{E}=-391.938316 \text { a.u. } \\
\boldsymbol{Z P \boldsymbol { V } \boldsymbol { E }}=164.58076 \mathrm{kcal} \mathrm{mol}^{-1} \\
\boldsymbol{G}_{298}=-391.710037 \text { a.u. }\end{array}$} \\
\hline
\end{tabular}




\section{References}

[1] Nicolai, S.; Sedigh-Zadeh, R.; Waser, J. J. Org. Chem. 2013, 78, 3783-3801.

[2] Lavinda, O.; Tran, V. T.; Woerpel, K. A. Org. Biomol. Chem. 2014, 12, 7083-7091.

[3] Avasthi, K.; Salomon, R. G. J. Org. Chem. 1986, 51, 2556-2562.

[4] Shook, C. A.; Romberger, M. L.; Jung, S.-H.; Xiao, M.; Sherbine, J. P.; Zhang, B.; Lin, F.-T.;

Cohen, T. J. Am. Chem. Soc. 1993, 115, 10754-10773.

[5] Krause, L.; Herbst-Irmer, R.; Sheldrick, G. M.; Stalke, D. J. Appl. Cryst. 2015, 48, 3-10.

[6] Sheldrick, G. M. Acta Cryst. A 2015, 71, 3-8.

[7] Sheldrick, G. M. Acta Cryst. C 2015, 71, 3-8.

[8] https://www.ccdc.cam.ac.uk/structures/

[9] Müller, P. Cryst. Rev. 2009, 15, 57-83.

[10] A. Thorn, B. Dittrich, G. M. Sheldrick Acta Cryst. A 2012, 68, 448-451.

[11] Parsons, S; Flack, H. D.; Wagner, T. Acta Cryst. B 2013, 69, 249-259.

[12] Gaussian 16, Revision C.01, Frisch, M. J.; Trucks, G. W.; Schlegel, H. B.; Scuseria, G. E.; Robb, M. A.; Cheeseman, J. R.; Scalmani, G.; Barone, V.; Petersson, G. A.; Nakatsuji, H.; Li, X.; Caricato, M.; Marenich, A. V.; Bloino, J.; Janesko, B. G.; Gomperts, R.; Mennucci, B.; Hratchian, H. P.; Ortiz, J. V.; Izmaylov, A. F.; Sonnenberg, J. L.; Williams-Young, D.; Ding, F.; Lipparini, F.; Egidi, F.; Goings, J.; Peng, B.; Petrone, A.; Henderson, T.; Ranasinghe, D.; Zakrzewski, V. G.; Gao, J.; Rega, N.; Zheng, G.; Liang, W.; Hada, M.; Ehara, M.; Toyota, K.; Fukuda, R.; Hasegawa, J.; Ishida, M.; Nakajima, T.; Honda, Y.; Kitao, O.; Nakai, H.; Vreven, T.; Throssell, K.; Montgomery, J. A., Jr.; Peralta, J. E.; Ogliaro, F.; Bearpark, M. J.; Heyd, J. J.; Brothers, E. N.; Kudin, K. N.; Staroverov, V. N.; Keith, T. A.; Kobayashi, R.; Normand, J.; Raghavachari, K.; Rendell, A. P.; Burant, J. C.; Iyengar, S. S.; Tomasi, J.; Cossi, M.; Millam, J. M.; Klene, M.; Adamo, C.; Cammi, R.; Ochterski, J. W.; Martin, R. L.; Morokuma, K.; Farkas, O.; Foresman, J. B.; Fox, D. J. Gaussian, Inc., Wallingford CT, 2016.

[13] Bruhn, T.; Schaumlöffel, A.; Hemberger, Y.; Pescitelli, G. SpecDis version 1.71, Berlin, Germany, 2017, http:/specdis-software.jimdo.com. 